QUARTERLY TECHNICAL PROGRESS REPORT

$$
\text { April, May, June } 1966
$$

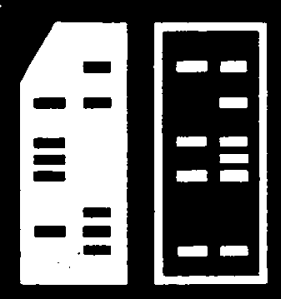

DEPARTMENT OF COMPUTER SCIENCE - UNIVERSITY OF ILLINOIS - URBANA, ILLINOIS 


\section{DISCLAIMER}

This report was prepared as an account of work sponsored by an agency of the United States Government. Neither the United States Government nor any agency Thereof, nor any of their employees, makes any warranty, express or implied, or assumes any legal liability or responsibility for the accuracy, completeness, or usefulness of any information, apparatus, product, or process disclosed, or represents that its use would not infringe privately owned rights. Reference herein to any specific commercial product, process, or service by trade name, trademark, manufacturer, or otherwise does not necessarily constitute or imply its endorsement, recommendation, or favoring by the United States Government or any agency thereof. The views and opinions of authors expressed herein do not necessarily state or reflect those of the United States Government or any agency thereof. 


\section{DISCLAIMER}

Portions of this document may be illegible in electronic image products. Images are produced from the best available original document. 


\section{QUARTERLY TECHNICAL PROGRESS REPORT}

April, May, June 1.966

\footnotetext{
LEGAL NOTICE

This report was prepared as an account of Government sponsored work. Neither the United States, nor the Commission, nor any person actung on behalf of the Comulssion:

A. Makes any warranty or representation, expressed or implied, with respect to the accuracy, completeness, or usefulness of the information contained in this report, or that the use of any information, apparatus, method, or process disclosed in this report may not Infringe of any information, apparatus
privately owned rights; or

privately owned rights; or B. Assumes any liabllities with respect to the use of, or for damages resul

use of any information, apparatus, method, or process disclosed in this report. As used in the above, "person acting on behalf of the Commission" Includes any em-
anter such employee or contractor of the Commission, or employee of such contractor prepares. disseminates, or provides access to, any Information pursuant to his employment or contract with the Commission, or his employment with such contractor.

Department of Computer Science

Uriversity of Illinois

Urbana, Illincis
} 
1. CIRCUIT RESEARCH PROGRAM . . . . . . . . . . . I I

Summary. . . . . . . . . . . . . . . . . . 1

1.01 Paramatrix System. . . . . . . . . . . 1

1.01.16.1 General. ............. 1

1.01.16.2 Slide-Scanner........... 1

1.01.16.3 Gap Filling. . . . ....... 2

1.02 Electro-Optical Work ........... 4

1.02.07 Analog Multiplier with Optic-

Electronic Effect......... 4

1.02.07.1 Introduction.......... 4

1.02.07.2 General Description. . . . . . 4

1.02.09.3 Error Analysis ......... 6

1.02.07.4 Limitation of Speed....... 8

$\begin{array}{ll}1.03 .01 & \text { Random Sequence Coding . . . . . . . . . . . } 9 \\ & 1.03 .01 .1 \text { RC Averaging Unit-Analysis. . . . } 9\end{array}$

1.03.01.2 Calculation of the Ripple for a

Periodic Pulse Sequence When Applied

1.03.01.3 Calculation of the Fluctuation

Voltage of a Random Pulse Sequence

When Applied to a Simple RC Low-

1.03.01.4 Results of the Experiments and Conclusions. . . . . . . . . 18

1.03.01.5 Multiplier and Divider Units . . . 19

Analog Storage.............. 21

1.06.02 PHASTOR--An Analog Memory. .... 21

1.06.02.01 Introduction........... . 21

1.06.02.2 Operation of PHASTOR ....... . 21

1.06.02.3 Brief Description of Circuits. . . . 22

1.06.02.4 Further Development. . . . . 23

2. HARDWARE SYSTEMS RESEARCH....................... 30

Summary. . . . . . . . . . . . . . . . . . 30

2.10 The Artrix System............ 30

2.10.811.1 Construction ........ 30

2.10.811.2 Pen Circuitry. . . . . . . . . 30

2.10.811.3 Video Levels............ 31

2.10.05.4 Processing Unit Logical Section. . . 31

2.10.09.5 Line and Circle Generator. . . . . 32

2.12 Pattern Transform Analysis .......... . 35

2.12.03.1 On-Line Fourier Transform System. . 35

2.12.03.2 Fourier Transform Optics..... 36

2.12.03.3 Transparency Plane........ 39 
TABLE OF CONTENTS (CONT'D)

Page

3. SOFTWARE AND TIME-SHARING SYSTEMS RESEARCH . . . . . . . 44

$3.1 \quad \mathrm{~B} \phi \phi \mathrm{TS} \ldots \ldots \ldots 45$

3.1.1 General ............ 45

3.1.2 Input-Output Routines ......... 45

3.2 Executive Programs for Time-Sharing. . . . . . 46

3.3 System Subroutines for Time-Sharing. . . . . . 46

3.4 SYSFBN and FRFSK. . . . . . . . . . 47

3.5 SORT-MERGE. . . . . . . . . . . . 47

3.6 Binary Card Loader. . ............ 48

3.7 SYSDSK, the Disk-Drum Routine .......... 48

3.8 Garbage Collection............ 50

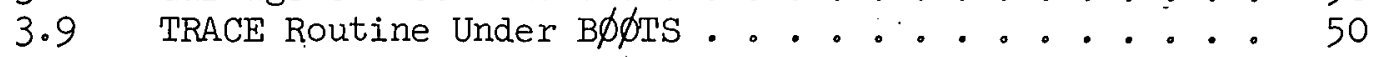

3.10 PDP-7 Programming .............. 51

3.11 SHфRTTRAN .................. 52

3.12 Graphical Display ................ 53

3.13 Time-Sharing Hardware ............ 53

3.13.1 Contest II.V. . . . . . . . . . 53

3.13.2 Bootstrap Using Disk Only . . . . . . 53

4. NUMERICAL METHODS, COMPUTER ARITHMETIC AND ARTIFICIAL

LANGUAGES. . . . . . . . . . . . . . . 54

4.1 Autonomous Arithmetic Unit Structures . . . . . . 54

4.1.1 Left-Directed Recoder .......... 54

4.1.2 Right-Directed Recoder......... 55

4.2 Relationship Between Arithmetic Structure and

Digital Probabilities ........... . 56

5. ILLIAC II SERVICE, USE, AND PROGRAM DEVELOPMENT. . . . . 58

5.1 ILLIAC II Program Development . . . ..... 58

5.1.1 System Program Development. . . . . . 58

5.1.2 FORTRAN, the ILLIAC II Compiler..... 59

$5: 1.3$ NICAP, the ILLIAC II Assembler, and

the NICAP MACRO PREPASS ....... 60

5.2 Library Programming ............ 61

5.2.1 ILLIAC II Library Development...... . 61

5.3 CalComp Digital Incremental Plotter ........ 65

5.4 Engineering Test Programming. . . . . . . . 66

5.5 Engineering Maintenance ........... . 67

5.5.1 ILLIAC II Engineering Log Summary - April,

May, June, 1966 ............ 67

5.5.2 Off-Line Equipment Requiring Repairs. . . 68

5.6 Scheduled Diagnostic Engineering. ....... 68

5.6.1 Maintenance .............. 68

5.6.2 Component Failures. . . . . . . 69

5.6 .3 Machine Modifications ........ 69

5.6.4 Channel Interface Checkout. ...... 70

5.6 .5 .. Transistor Replacements .......: 70

5.6.6 Causes for Computer Down Time...... 70 
TABLE OF' CONTENTS (CONT'D)

Page

5.7 Log Summaries ............. . . 71

5.7.1 ILLIAC II Use . . . . . . . . . 71

5.7.1.1 Summary of Use. ...... . 71

5.7.1.2 Summary of Machine Errors. . 74

5.7.2 IBM 1401, 1402, 1403 Use. . . . . 78

5.7.2.1 Summary of Use. . . . . . 78

5.7.2.2 Summary of Machine Errors . . 79

6. : IBM SERVICE: USE, AND :PROGRAM DEVELOPMENT: 。 .

6.1 New Routines. . .............. 80

6.2 Log Summaries ................ . . 83

7. PROBLEM SPECIFICATIONS . . . . . . . . . . . . . 107

7.1 Research Problem Specifications......... . 107

7.2 Instructional Problem Specifications. . . . . . . 167

7.3 Blanket Class Problem Specifications. . . . . . 168

8. GENERAL LABORATORY INFORMATION . . . . . . . . . 170

8.1 Personnel................... 170

8.2 Bibliography. ............... . . . . 171

8.3 Numerical Analysis. . . . . . . . . . . 173

8.4 Colloquia.......................... 174

8.5 Drafting. ................. . . . 175

8.6 Shops' Production............ 175 


\section{1: CIRCUIT RESEARCH PROGRAM}

(Supported in part by the Office of Naval Research under Contract Nonr$1834(15)$.

\section{$\underline{\text { Summary }}$}

The Paramatrix System is moving into its second phase with the design of a slide-scanning input, by Larry Ryan, to replace the potentiometers of phase one.

A novel analog multiplier using photo-emitting diodes and photo-sensitive resistors forms the basis of Dick Koo's report on ElectroOptical Work.

Following up his last report of a DC averaging unit for Random Sequence Coding, Chushin Afuso discusses experiments. with an $\mathrm{AC}$ averaging technique and compares the results of the two methods.

Finally, a totally new approach to Analog Storage, proposed by W. J. Poppelbaum, D. Aspinall and M. Faiman, is described by Ed Carr and Larry Wallman. Their report discusses the prototype system--PHASTOR-which has already been built and is working reliably.

\subsection{Paramatrix System}

\section{$: 1.01 .16 .1$ General}

A preliminary design of a slide-scanner input for Paramatrix is proposed. It consists of a CRT flying-spot scanner, photo-cell, counters, D/A converters, and associated logic circuitry.

\subsection{1 .16 .2 Slide-Scanner}

Figure 1 illustrates the slide-scanner system without the gapfilling circuits. The horizontal displacement of the CRT beam is con: trolled by the "horizontal" output, $x_{i j}$, of the Transformer. The vertical displacement is controlled. by a highly linear sweep circuit.

For each $x_{i j}$ made available, the CRT beam sweeps the appropriate column on the slide, completing the sweep in one Paramatrix clock time: 
At the start of each sweep four 5-bit counters (four being equal to the maximum number of profiles) begin counting in synchronism with the sweep, so that the contents of the counters at any time are directly proportional to the vertical height of the CRT beam.

The change in intensity of light behind the slide is detected by a high speed photo-cell. The output of the photo-cell is shaped and used to stop successive counters as line segments are scanned on the slide. It is clear that an auxiliary counter is necessary to keep track of the number of line segments scanned and to gate a "stop" signal to the appropriatecounters. As the counters are stopped their contents are read out by D/A converters and compared with the "vertical" Transformer output $\mathrm{y}_{i j}$. The analog comparison is accomplished as in the existing system, by the Coincidence Unit.

\subsubsection{Gap Filling}

A method of filling in gaps, based on repeated sweeps of any given neighborhood of the slide, is currently being developed. Details: will be given in the next report. 

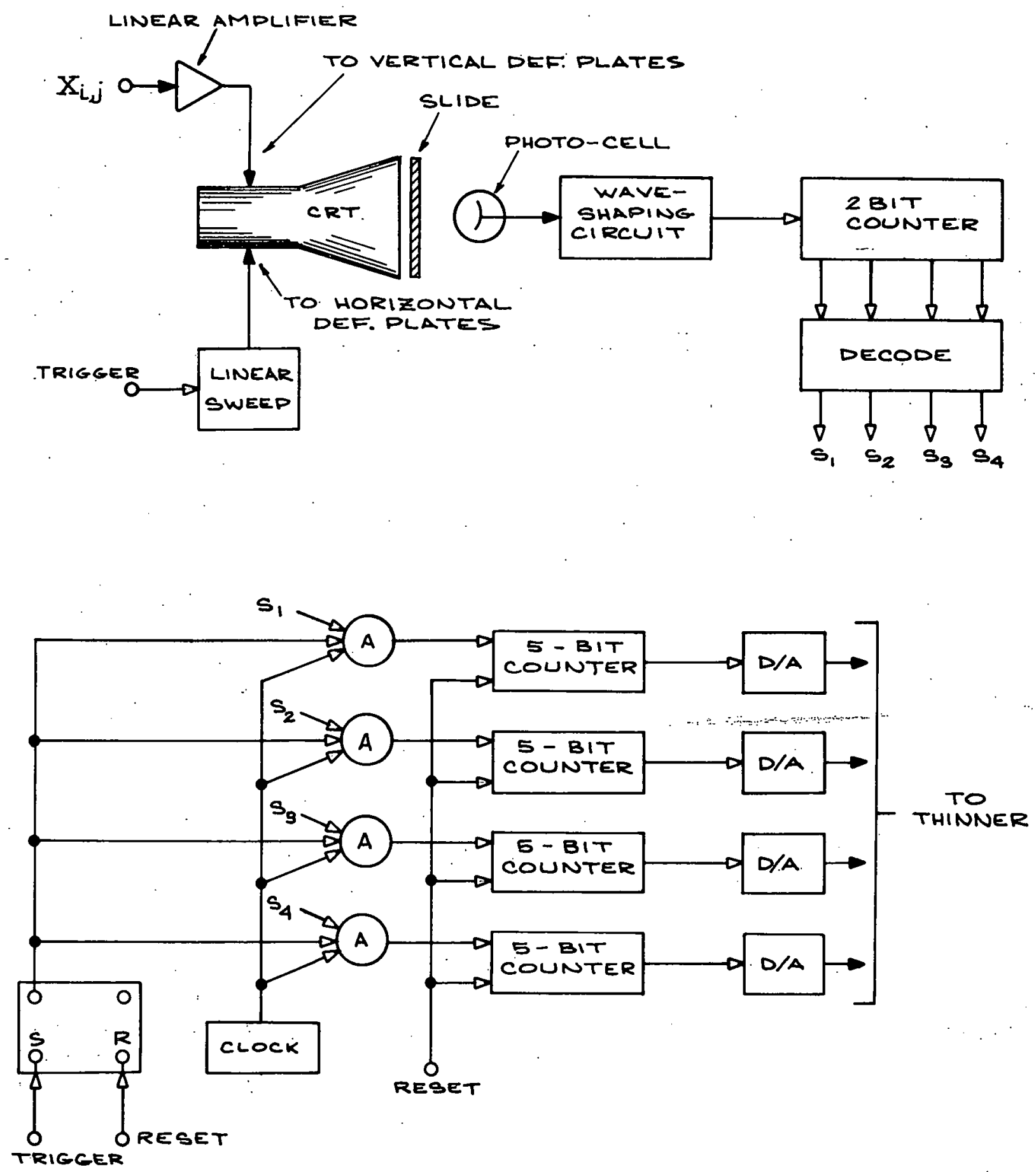

Figure 1. Slide-Scanner Without Gap-Filling Circuits. 


\subsection{Electro-Optical Work}

\subsubsection{Analog Multiplier with Optic-Electronic Effect}

\subsubsection{Introduction}

With the goal of high speed analog computing or a hybrid computation system, the development of a fast analog multiplier with reasonable accuracy is necessary. A multiplier using the principle of crossed-field deflection has been claimed to have an accuracy of about $1 \%$ up to a frequency of several kc/s. A "quarter-square" multiplier has a response up to. $50 \mathrm{kc} / \mathrm{s}$ with an accuracy around $0.3 \%$. The one which has been described as having exceptional bandwidth is a parabolic law beam-deflector which has a bandwidth of $90 \mathrm{kc} / \mathrm{s}$ and an overall accuracy of $0.5 \%$.

In the following sections a multiplier using a photo-conductive cell will be described. The new circuit has the advantage of larger bandwidth without loss of accuracy. A feasibility model has been built and a prototype is at present being constructed.

\subsubsection{General Description}

It is well known that the conductance of a photoconductor is directly proportional to the incident light intensity. The steady state resistance $R_{p}$ of such a specimen can be written as:

$$
R_{p}=\frac{R_{O S}}{I+\alpha I}
$$

where

$$
\begin{aligned}
R_{0}= & \text { dark resistance } \\
I= & \text { light intensity } \\
\alpha= & \text { coefficient depending upon photocell } \\
& \text { material and frequency of incident } \\
& \text { light. }
\end{aligned}
$$

Two matched photoconductors are incorporated into a circuit as shown in Figure 1. The analog voltages to be multiplied are $\mathrm{x}$ and $\mathrm{y}$, and $\mathrm{x}$ is fed through non-inverting and inverting drivers to the photoemitting 
diodes to produce changes of their output light intensities corresponding to $+x$ and $-x$ respectively. Then the resistances of the photoconductors will be:

$$
R_{p l}=\frac{R_{0}}{1+\alpha\left(I_{0}-k x\right)} ; \quad R_{p 2}=\frac{R_{0}}{I+\alpha\left(I_{0}+k x\right)}
$$

where $\quad I_{0}=$ biasing output of photoemitting diode $\mathrm{k}=$ conversion factor between $\mathrm{x}$ and output light intensity.

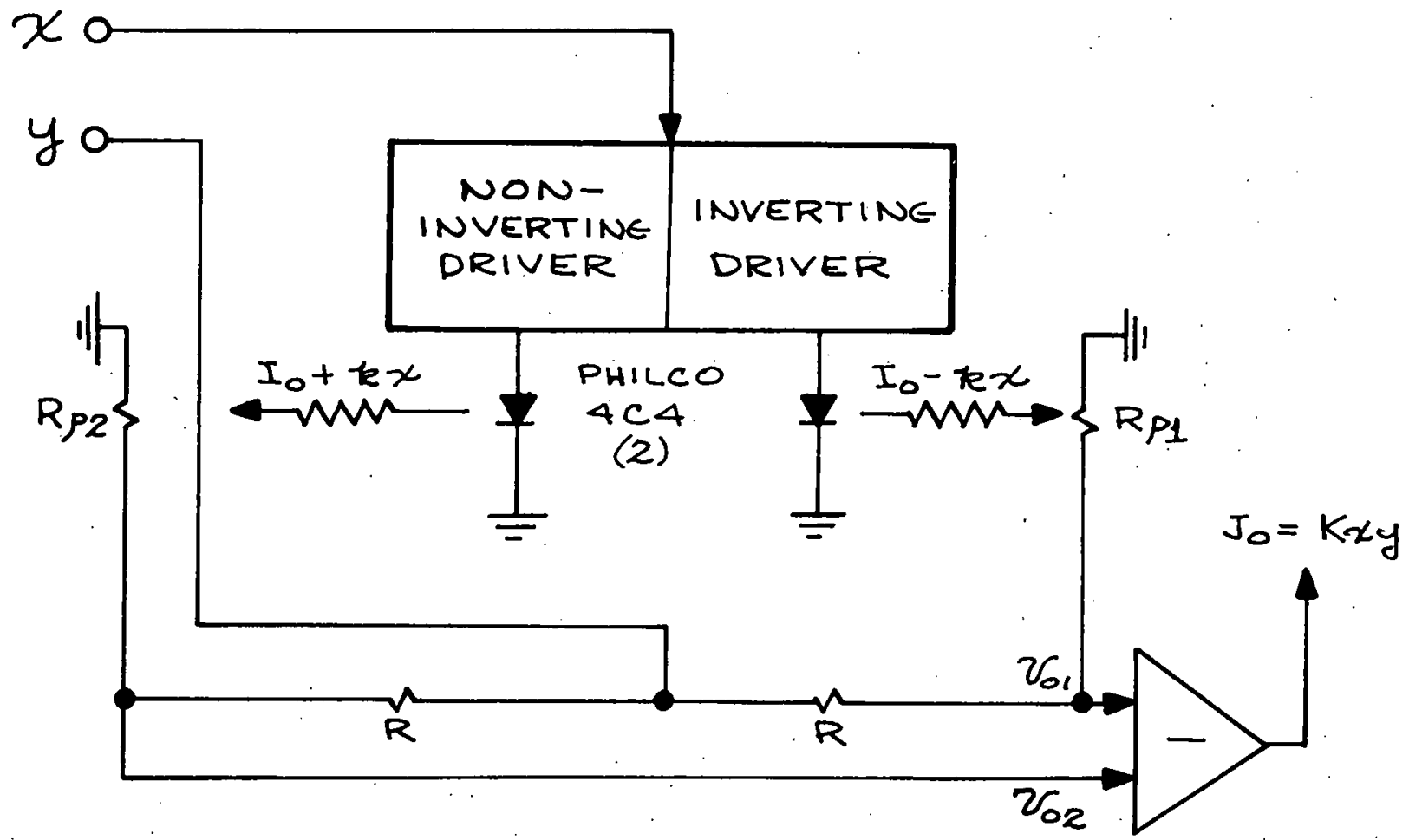

Figure 1. Circuit Diagram of Photoconductor Multiplier.

With simple circuit analysis, it can be shown that

$$
\mathrm{V}_{\mathrm{O} 1}-\mathrm{V}_{02}=\frac{\mathrm{R}_{0}}{\mathrm{R}} \frac{2 \alpha k}{\left(1+\alpha I_{0}+\frac{\mathrm{R}_{0}}{\mathrm{R}_{-}}\right)^{2}-(\alpha k x)^{2}} \cdot x y
$$


Since it is possible to select the circuit elements such that:

$$
1+\alpha . I_{0}+\frac{R_{0}}{R . .} \gg|\alpha k x|
$$

Equation (3) can be approximated as

$$
\mathrm{V}_{0}=\mathrm{V}_{01}-\mathrm{V}_{02}=\frac{\mathrm{R}_{0}}{\mathrm{R}} \frac{2 \alpha \mathrm{k}}{\left(1+\alpha I_{0}+\frac{\mathrm{R}_{\mathrm{O}}}{\mathrm{R}_{r}}\right)^{2}} \cdot \mathrm{xy}=\mathrm{Kxy}
$$

Thus, it is clear that if the signals $V_{01}$ and $\dot{V}_{02}$ are picked up by a:differential amplifier, its output will be the product of $x$ and $y$ with a scale factor.

\subsubsection{Error Analysis}

There are several factors that will affect the accuracy of the output. Since all components can be carefully matched within $0.01 \%$, there being only a few of them, the error introduced by components is therefore in the acceptable range. The stability and accuracy of the drivers play àn important role in the overall accuracy. Their accuracy can be achieved. by conventional circuit techniques. One error factor which is inherent in the system is the approximation error from Eq. (3) to Eq. (4). This factor will be analyzed as follows:

Let $V_{e}$ be the error voltage, equal to the difference between Eq. (3) and Eq.(4). Then

$$
\mathrm{v}_{\mathrm{e}}=\mathrm{v}_{0} \frac{(\dot{\alpha} \mathrm{kx})^{2}}{\left(i+\alpha I_{0}+\frac{\mathrm{R}_{\mathrm{g}}}{\mathrm{R}_{\mathrm{r}}}\right)(\alpha \mathrm{kx})^{2}}
$$


Therefore the relative error is

$$
e=\frac{V_{e}}{V_{0}}=\frac{(\alpha k x)^{2}}{\left(I+\alpha I_{0}+\frac{R_{0}}{R_{i}}\right)^{2}-(\alpha k x)^{2}}
$$

From this equation, it is obvious that the relative error is independent of $y$ and is a maximum when $x$ is a maximum. So

$$
e_{\max }=\frac{\left(\alpha k \dot{x}_{m}\right)^{2}}{\left(1+\alpha I_{0}+\frac{R_{0}}{R_{n}}\right)^{2}-\left(\alpha k x_{m}\right)^{2}}
$$

Eq. (7) reveals that $e_{\max }$ might be reduced arbitrarily by increasing $\frac{R_{0}}{R_{1}}$; but this cannot be done due to the fact that the output signal will also be reduced. To find an optimum condition, assume that the smallest value of $\mathrm{x}, \mathrm{y}$, (other than, 0 ) to be distinguished is 1 volt and that the minimum signal detectable by the differential amplifier is Bmv. Assume also that the maximum change of light intensity from the photoemitting diodes is $a I_{0}$ with $a \leq 1$. Then $\mathrm{kx}_{\mathrm{m}}=\mathrm{aI_{0 }}$ and

$$
\beta=\frac{200 \alpha a I_{0}}{\left(I+\alpha I_{0}+\frac{R_{0}}{R_{r}}\right)^{2}} \cdot \frac{R_{\delta}}{R_{t}}
$$

From Eq. (7) and Eq. (8), it follows that

$$
\beta=\frac{200 e_{m}}{1+\dot{e}_{m}}\left\{\sqrt{\frac{I+\bar{e}_{m}}{e_{m}}}-\frac{1}{a \dot{\alpha}}-\frac{1}{\alpha I_{0}}\right\}
$$


As an example, let $e_{m}=0.1 \%, \alpha I_{0}=10, a \cdot \alpha:=0.5$; then $\beta=5.88$, $\frac{\mathrm{R}_{\mathrm{O}}}{\mathrm{R}}=147$. Therefore, it is concluded that the higher the sensitivity of the differential amplifier, the higher the accuracy can be.

\subsubsection{Limitation of Speed}

The primary limit imposed on this circuit.is the relaxation time of the photoconductor. It is well known that this is not necessarily a constant. At low levels, namely, when the excess carrier density is small compared with the equilibrium density, the time constant is dominated by linear recombination and therefore can be regarded as constant. However, at the level where the excess carrier density is larger than the equilibrium density, the time constant will be dominated by quadratic recombination and impact recombination, as well as the mechanism of trapping. The time constant is thus a function of excess carrier density. Usually, the higher the excess carrier density, the smaller the time constant. Also, as a result of non-linear recombination, the fall time is larger than the rise time. The phenomena are illustrated in Figure 2.

In the present case, in order to increase the frequency response, it is desirahle to operate the photoconductors at high level with a large bias $I_{0}$. Then since $\Delta I_{0}(x) \ll I_{O}$, the fall time constant corresponding to $I_{0}$ will determine the speed of the circuit.

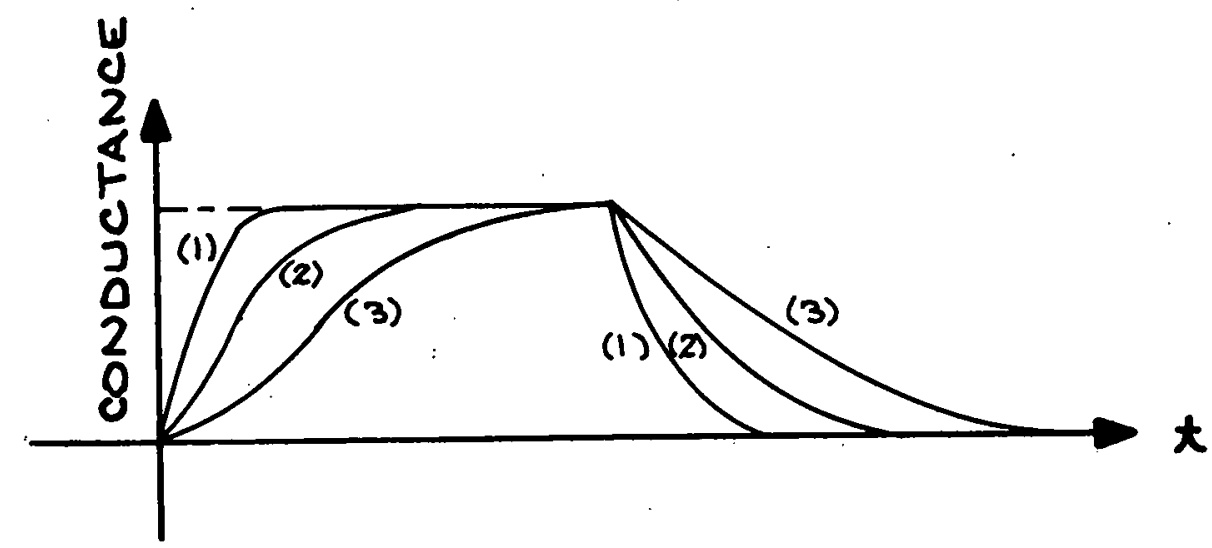

Figure 2. Normalized Square Light Pulse Response. Intensity: I(I) $>I(2) \cdot I$ I. $(3)$. 
A photoconductor with time constant around lusec is now obtainable. Therefore, the frequency limit may be expected to be about $200 \mathrm{kc}$.

\subsubsection{Random Sequence Coding}

In the last quarterly report it was mentioned that there were two ways of taking the average of a random pulse sequence. One of these, the digital averaging method was described, as well as the experimental results.

This time a simple RC. low-pass filter is discussed as an example of the analog averaging method. Comparison between characteristics of both cases indicates that the RC low-pass filter is more attractive in practice.

\subsubsection{RC Averaging Unit--Analysis}

The output of the random pulse sequence generator is taken from the collector of a non-saturating transistor. The collector current, therefore, is considered to be approximately a current source. In this case the simplest RC low-pass filter is obtained by connecting a capacitor across the autput resistor. The idealized circuit for this case is shown in Figure 1.

In order to estimate the effect of the $\mathrm{RC}$ averaging circuit we proceed as follows.

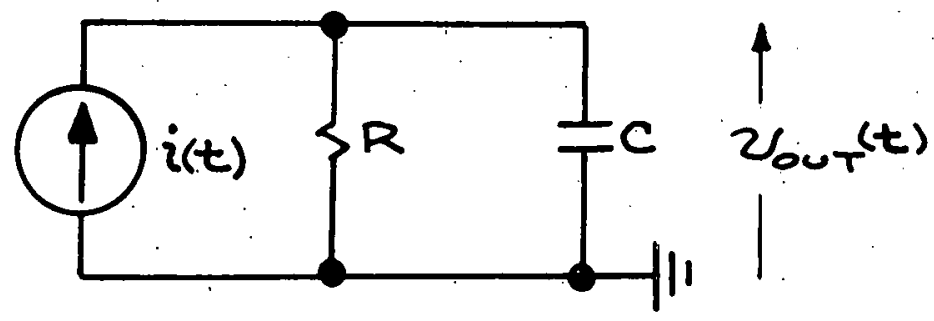

$$
i(t) \text { : "'- Random Puilse Sequence "Current Generator. }
$$

Figure 1. Simplest RC Averaging Circuit for a Current Source. 
A random pulse sequence may be regarded as a frequencymodulated pulse sequence in which the frequency (repetition rate) varies with time at random about the average frequency. The distribution of frequency is given by the Poisson distribution because of the inherent nature of the random pulse generator. For sufficiently large values of the frequency the probability density is given by the normal density function as shown in Figure $2 . f_{\ell}$ and $f_{u}$ are the practical minimum and maximum frequencies in the sense that $95.6 \%$ of the whole distribution lies in the range $\left(f_{\ell}, f_{u}\right)$. The lower limit of the voltage fluctuation at the output is related to $f_{l}$ and the upper iimit to $f_{u}$. Therefore, the voltage fluctuation due to the fluctuation of the pulse repetition rate may be calculated from the difference between the output voltage for a periodic pulse sequence with $f_{u}$ and that for a periodic pulse sequence with $f_{\ell}$.

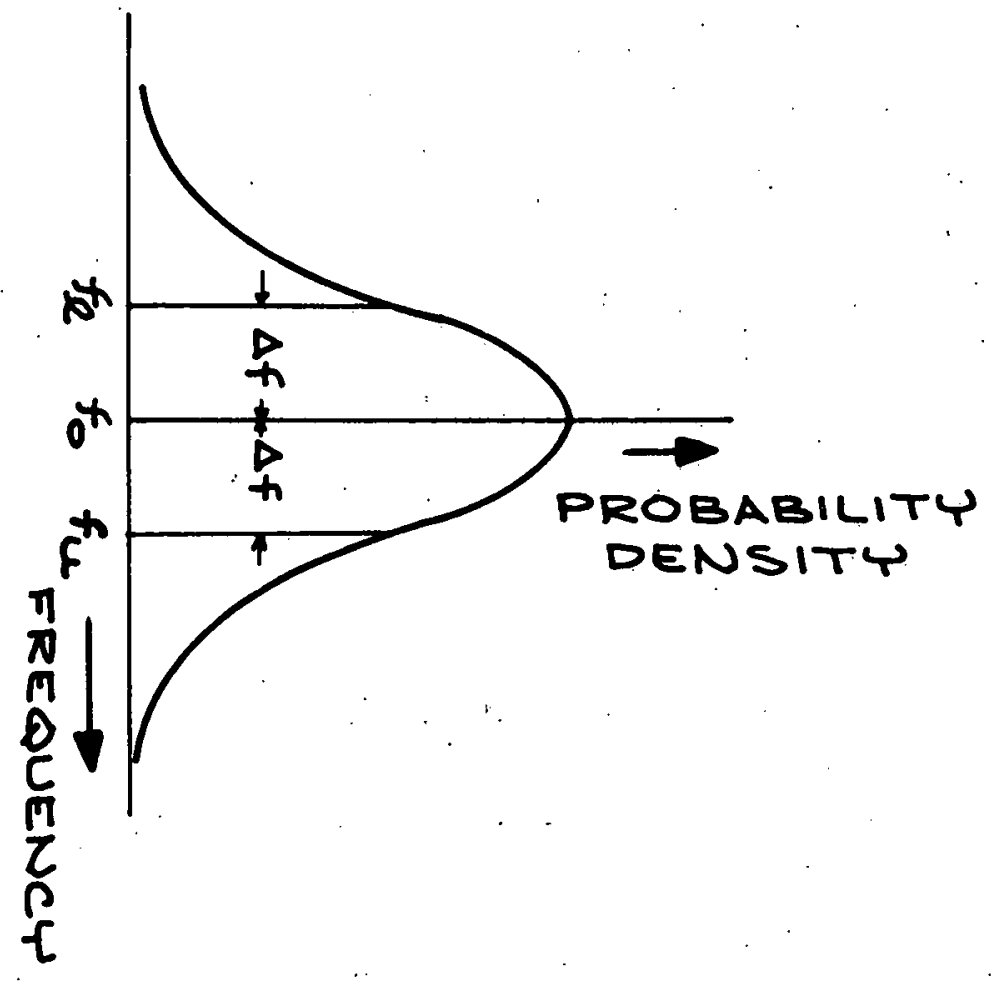

$f_{0}=$ average frequency; $\Delta f=$ standard deviation;

$f_{l}=f_{0}-\Delta f ; \quad f_{u}=f_{0}+\Delta f$

Figure 2. Probability Density of the Frequency of the Random Pulse Sequence. 


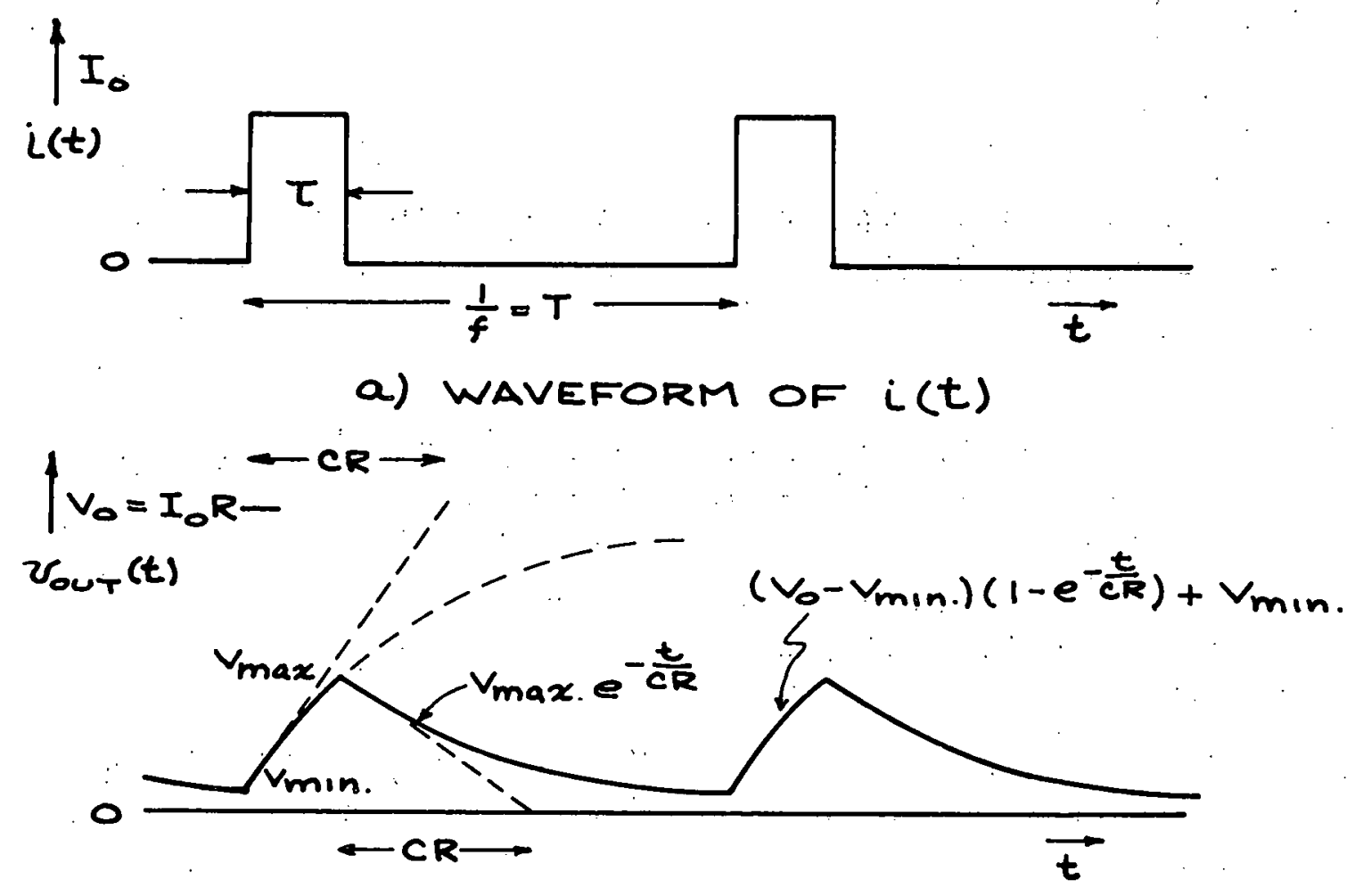

b) WAVEFORM OF $\tau_{\text {OUT }}(\tau)$

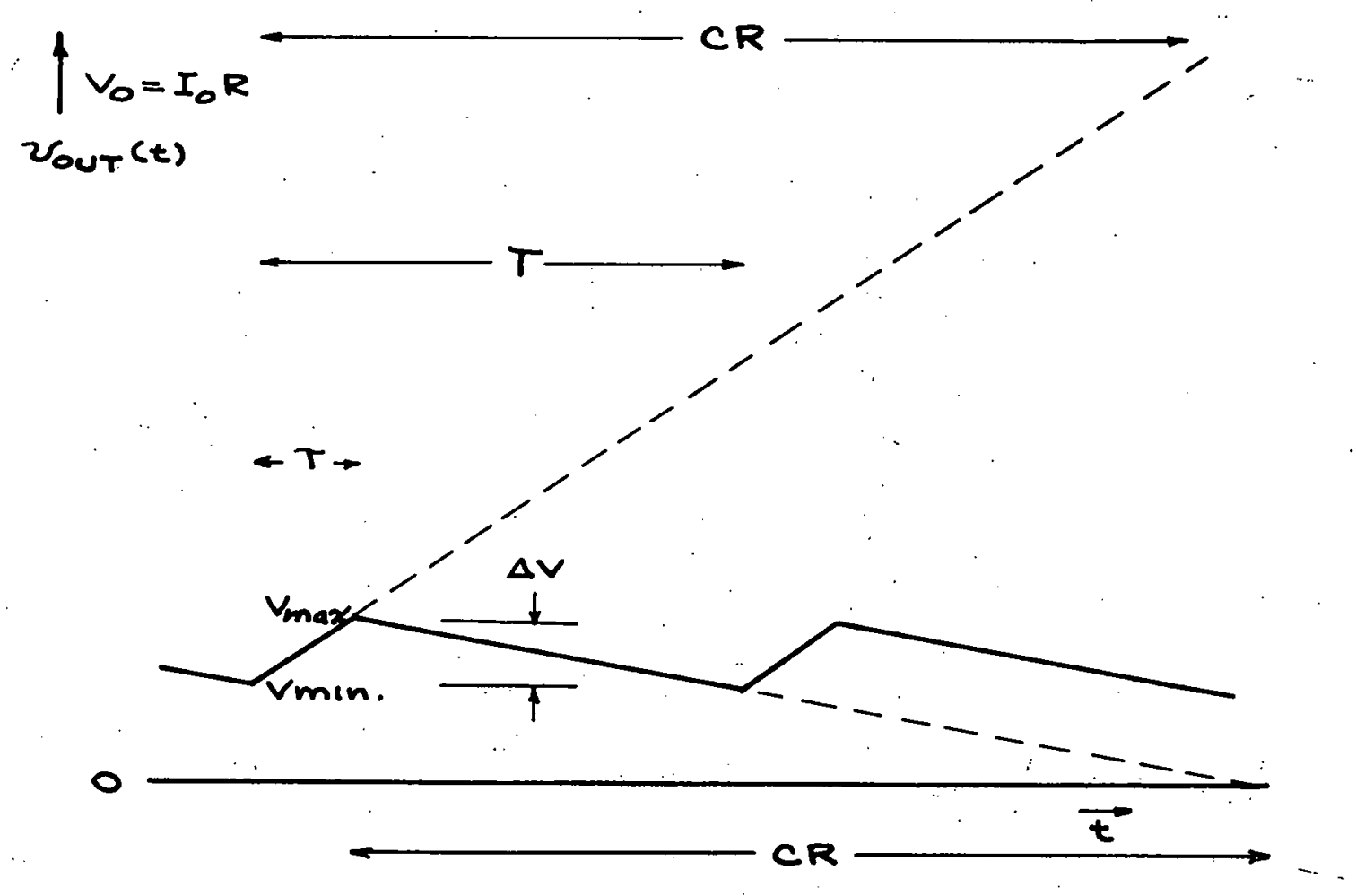

c) WAVEFORM OF $\tau_{\text {OUT }}(\tau)$ WHEN $\tau$,
$T<\angle C R$

Figure 3. Waveforms of $i(t)$ and $v_{\text {out }}(t)$ : 
First let us consider the effect of the RC low-pass filter for a periodic pulse sequence.

1.03.01.2 Calculation of the Ripple for a Periodic Pulse Sequence When Applied to a Simple RC Low-Pass Filter.

Let us consider the eircuit shown in Figure 1 , where now $i(t)$ is a periodical pulse current generator. Assuming that $i(t)$ has the waveform as shown in figure $3(a)$, then the output voltage $v_{\text {out }}(t)$ has the waveform as shown in Figure $3(\mathrm{~b})$.

If $\tau \ll<R$, and $T \ll<C$, the exponential term may be approximated linearly. The waveform in this case is shown in Figure $3(\mathrm{c})$. For the charging process:

$$
\frac{V_{\max }-V_{\min }}{\tau}=\frac{V_{0}-V_{\min }}{C R}
$$

For the discharging process:

$$
\frac{V_{\max }-V_{\min }}{T-\tau}=\frac{V_{\text {max }}}{C R}
$$

From (I) and (2), $V_{\max }$ and $V_{\min }$ are solved and the results are:

$$
V_{\max }=\frac{\mathrm{CR} \tau}{\mathrm{CRT}+\tau^{2}} \mathrm{~V}_{\mathrm{O}}, \quad \mathrm{V}_{\min }=\frac{(\mathrm{CR}+\tau-\mathrm{T}) \tau}{\mathrm{CRT}+\tau^{2}} \mathrm{~V}_{\mathrm{O}}
$$

Then the ripple voltage, $\delta \mathrm{V}$, is given by

$$
\delta V=V_{\max }-V_{\min }=\frac{(T-\tau) \tau}{\operatorname{CRT}+\tau^{2}} V_{O}
$$


1.03.01.3 Calculation of the Fluctuation Voltage of a Random Pulse Sequence When Applied to a Simple RC Lod-Pass Filter.

The practical upper limit of the voltage fluctuation is obtained when a periodic pulse sequence with the repetition rate $f_{u}$ is applied as the : input. $\therefore$. Conversely, the lower limit is obtained by applying a periodic pulse sequence with $f_{\ell}$. This is shown in Figure 4. Hence we have:

$$
\text { Fluctuation voltage: } \Delta V=V_{\text {umax }}-V_{\text {lmin }} \text {. }
$$

Let $V_{\text {uav }:}$ = average voltage for a periodic pulse sequence with $f_{u}$.

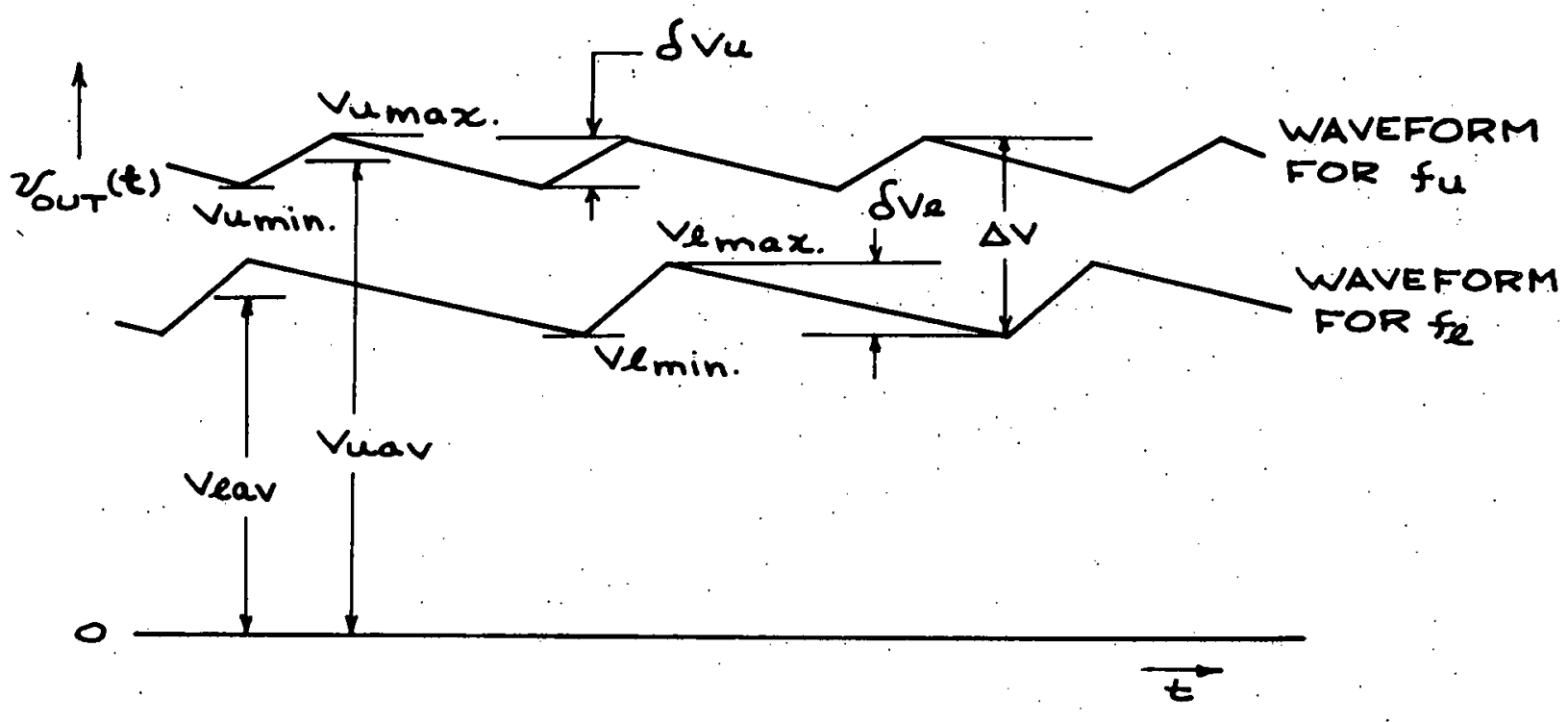

Figure 4. Waveforms of $V_{\text {out }}(t)$ for Periodic Pulse Sequences with $f_{u}$ and $f_{\ell}$.

Then $\mathrm{V}_{\max }=\mathrm{V}_{\text {uav }}+\frac{1}{2} \delta \mathrm{v}_{\mathrm{u}}$

$$
\begin{aligned}
& =V_{0} \tau f_{u}+\frac{1}{2} \frac{\left(T_{u-w}-\tau\right) \tau}{C R T_{u}+\tau^{2}} V_{0} \\
& =V_{0} \tau\left[f_{u}+\frac{1}{2} \frac{T_{u}-\tau}{\mathrm{CRT}+\tau^{2}}\right]
\end{aligned}
$$


Where $T_{u}=\frac{\dddot{I}}{f_{u}}$

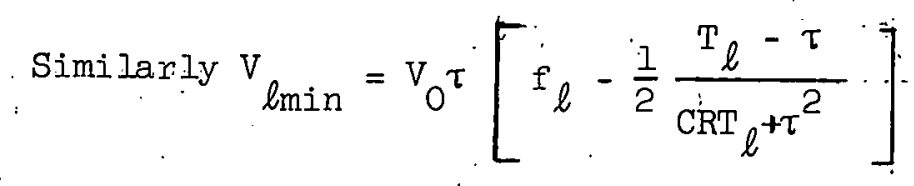

with $\quad \mathrm{T}_{\ell}=\frac{\dot{1}}{\mathrm{f}_{\ell}}$

Therefore

$$
\Delta V=V_{0} \tau\left[f_{u}-f_{\ell}+\frac{1}{2}\left\{\frac{T_{u}-\tau}{\mathrm{CRT}_{u}+\tau^{2}}+\frac{T_{\ell}-\tau}{\mathrm{CRT}_{\ell}+\tau^{2}}\right\}\right]
$$

$f_{u}$ and $f_{l}$ are determined by the average frequency, $f_{O}$, and the standard deviation; $\Delta f$. Let $f_{0}=$ average frequency in $\mathrm{cps}, \mathrm{T}_{\mathrm{c}}=$ counting duration in seconds. Then for the Poisson distribution:

Standard deviation of the number of pulses within $\mathrm{T}_{c}=\sqrt{\mathrm{f}_{\mathrm{O}} \mathrm{T}_{c}}$

and hence

Practical maximum number of pulses

$$
\begin{aligned}
\text { within } T_{c} & =f_{O} T_{c}+\sqrt{f_{O} T_{c}} \\
& =F_{O} T_{c}\left(1+\frac{1}{\sqrt{f_{0} T_{c}}}\right)
\end{aligned}
$$


or

$$
\begin{aligned}
& \text { Practical maximum frequency in cps: } f_{u} \\
& =f_{0}\left(1+\frac{1}{\sqrt{f_{0} T_{c}}}\right)
\end{aligned}
$$

Similarly

$$
\therefore f_{\ell}=f_{0}\left(1-\frac{1}{\sqrt{f_{0} T_{c}}}\right)
$$

Since counting the pulses does not take place actually, it is not possible to define a definite value for $\mathrm{T}_{c}$. However, for the purpose of estimating the fluctuation, it would seem reasonable to take the value of $C R$ as. $T_{c}$, $i . e .$, we set

$$
\mathrm{T}_{\mathrm{c}}=\mathrm{CR}
$$

Substituting (7), (8), and (9) into (6), we have

$\Delta \mathrm{V}=2 \mathrm{v}_{\mathrm{O}} \tau \mathrm{f}_{\mathrm{O}}\left[\frac{1}{\sqrt{\mathrm{CRf}}}+\frac{1}{4 \mathrm{CRf}}\left\{2-\left(1+\frac{\tau}{\mathrm{CR}}\right)_{0}\left(\frac{1}{\frac{\mathrm{T}}{\tau}+\frac{\tau}{\mathrm{CR}}}+\frac{1}{\frac{\mathrm{T}}{\tau}+\frac{\tau}{\mathrm{CR}}}\right)\right\}\right.$

Since $\frac{\tau}{\mathrm{CR}} \ll 1<\frac{\mathrm{T}_{u}}{\tau} ; \frac{T_{\ell}}{\tau}$, this equation simplifies to

$$
\begin{gathered}
\Delta V=2 V_{O} \tau f_{0}\left[\frac{1}{\sqrt{C R f_{0}}}+\frac{1}{4 C R f_{0}}\left\{2-\tau\left(\frac{1}{T_{u}}+\frac{I}{T_{l}}\right)\right\}\right] \\
\quad=2 v_{0} \tau f_{0}\left[\frac{1}{\sqrt{C R f_{0}}}+\frac{I-f_{0} \tau}{2 C R f_{0}}\right]
\end{gathered}
$$


Let us define the relative fluctuation, $\Delta$, by

$$
\Delta=\frac{\frac{1}{2} \cdot \Delta V}{V_{a v}}
$$

where $\mathrm{V}_{\mathrm{av}}=$ average voltage of the random pulse sequence

$$
=V_{0} \tau f_{0}
$$

Then from (10) and (11), we finally have

$$
\Delta=\frac{1}{\sqrt{\mathrm{CRf_{0 }}}}+\frac{1-\mathrm{f}_{0} \tau}{{ }^{2 \mathrm{CRf}} \mathrm{O}_{1}}
$$

on the assumptions:

$$
\begin{aligned}
& \tau \ll \mathrm{CR} \\
& \mathrm{T}_{\ell} \ll \mathrm{CR}
\end{aligned}
$$

In (12), the first term is due to the fluctuation of the repetition rate, and the second term is due to the ripple which we calculated for a single periodic pulse sequence. 


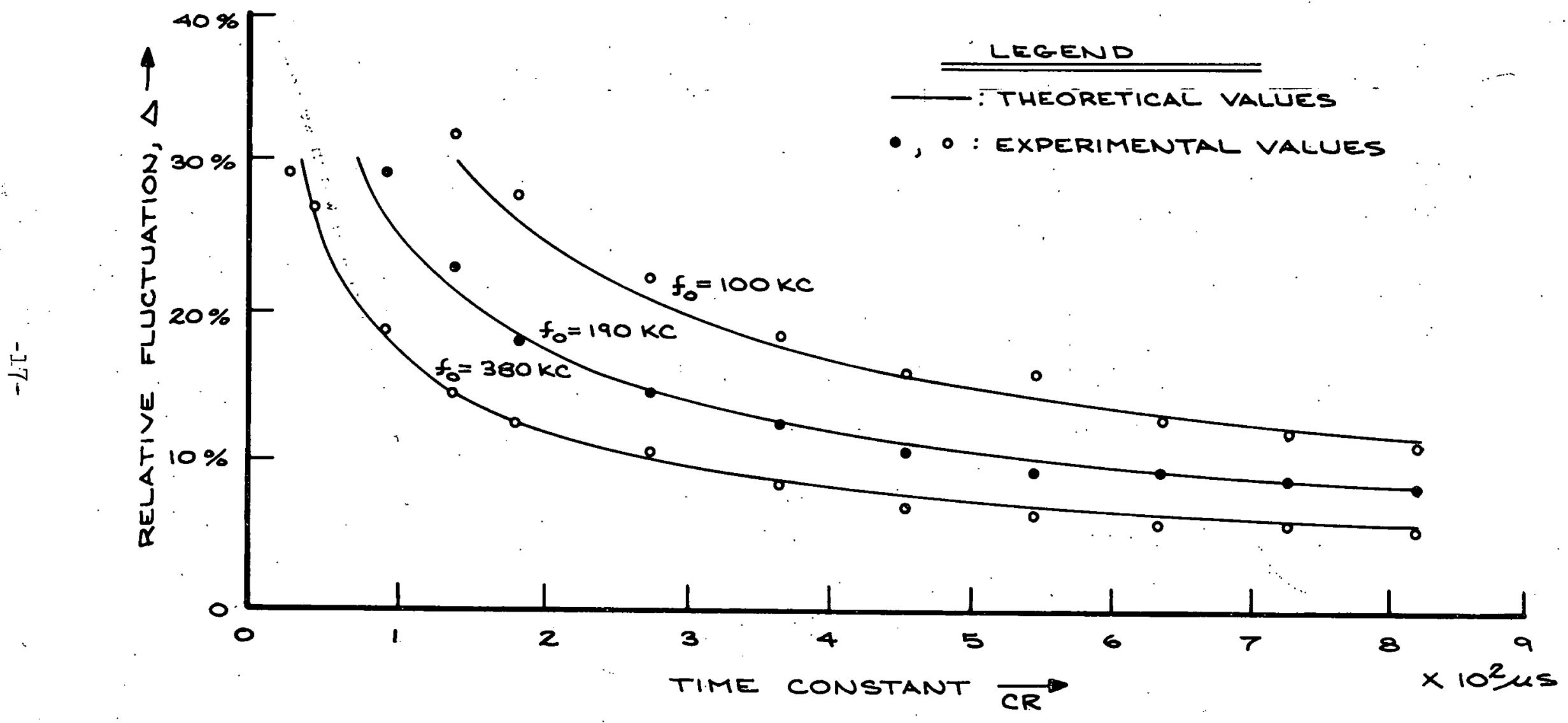

Figure 5. Relative Fluctuation as a Function of the Time-Constant. 


\subsubsection{Results of the Experiments and Conclusions}

In Figure $5 \Delta$ is plotted as a function of the time-constant

of the filter, CR, taking $f_{0}$ as a parameter. The results of the experiments are also plotted, and they agree with the theoretical values fairly well. The fluctuation voltage was measured by means of the oscilloscope and the average voltage was read on the D'Arsonvaltype voltmeter.

In the experiments

$$
\begin{aligned}
\mathrm{R} & =910 \text { ohms } \\
\tau & =0.7 \mathrm{\mu s} \\
\mathrm{V}_{0} & =9.0 \text { volts }
\end{aligned}
$$

are chosen. Let us check whether the assumptions are satisfied.

For the range $\mathrm{CR}>30 \mu \mathrm{s}$, (13), is satisfied.

Tio check (14)

$$
\begin{aligned}
\text { i) Taking } & \mathrm{f}_{0}=380 \mathrm{kc} / \mathrm{s} \text { and } \mathrm{CR}=30 \mu \mathrm{s} \\
\text { then } & \mathrm{T}_{\ell}=3.7 \mu \mathrm{s} \ll 30 \mu \mathrm{s} \\
\text { ii) Taking } & \mathrm{f}_{0}=190 \mathrm{kc} / \mathrm{s} \text { and } \mathrm{CR}=70 \mu \mathrm{s} \\
\text { then } & \mathrm{T}_{\ell}=7.3 \mu \mathrm{s} \ll 70 \mu \mathrm{s} \\
\text { iii) Taking } & \mathrm{f}_{0}=100 \mathrm{kc} / \mathrm{s} \text { and } \mathrm{CR}=140 \mu \mathrm{s} \\
\text { then: } & \mathrm{T}_{\ell}=14 \mu \mathrm{s} \ll 140 \mathrm{\mu s}
\end{aligned}
$$

Thus the assumptions are valid within the plotted range.

For a given time-constant the relative fluctuation varies as a function of the average frequency. As the average frequency decreases the fluctuation increases. Since the accuracy of the analog quantity, which is proportional to the average frequency, is limited by the fluctuation, the lower limit of the analgg 
quantity is bounded by the accuracy. Quantitative investigations for wide frequency ranges are in progress.

In Figure 6. the results of the experiments for the RC filter are compared with those of the digital averaging unit which were described in the last quarterly report. The time-constant and the counting duration are taken on a common scale. It is clearithat the $\mathrm{RC}$ filter has advantages in both effectiveness and cost.

\subsubsection{Multiplier and Divider Units}

Experimental circuits of the multiplier and the divider have been completed. Tests show that they work more or less as predicted by theory; however, quantitative data are not available yet. 


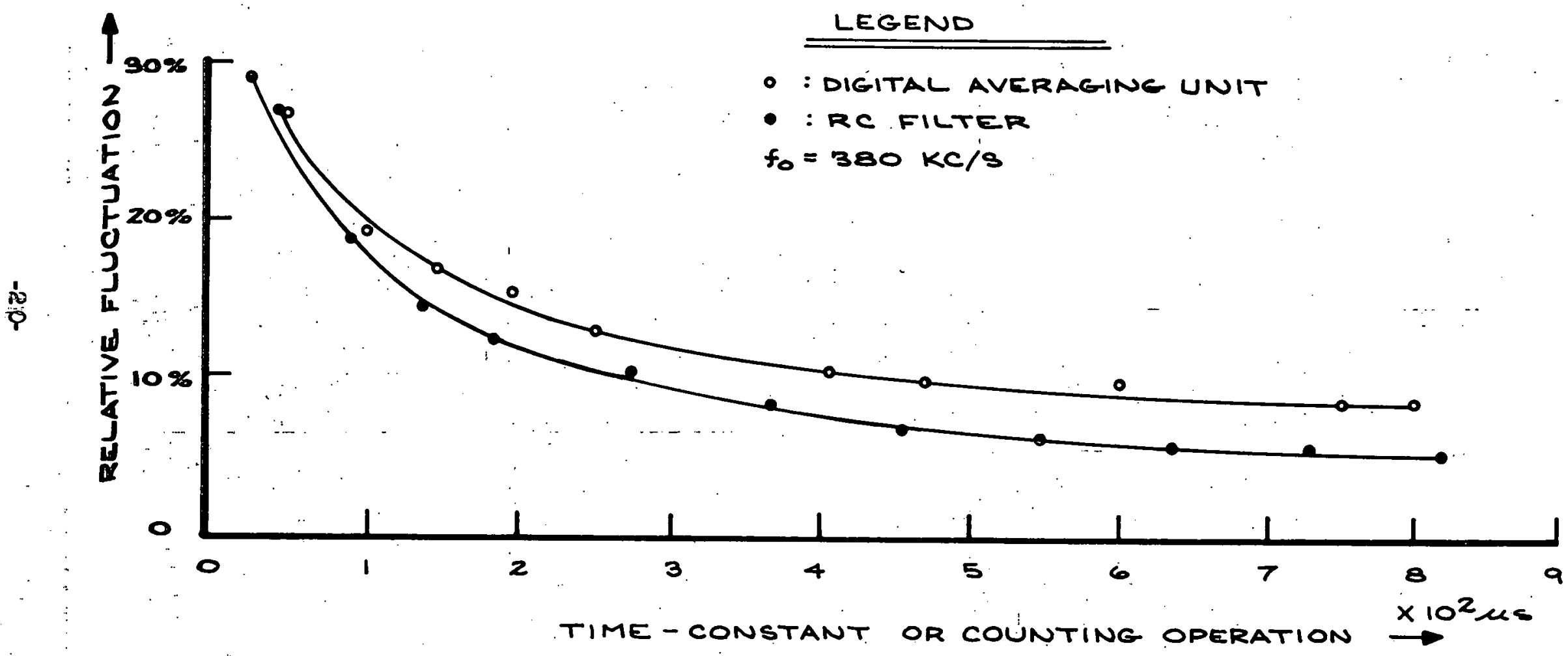

Figure 6. Relative Fluctuations as Functions of Time-Constant or Counting Duration. 


\subsection{Analog Storage}

\subsection{6 .02 PHASTOR--An Analog Memory}

\subsubsection{Introduction}

A novel method for analog storage using a delay line technique has been probosed by W. J. Poppelbaum, D. Aspinall, and M. Faiman. The storage element is actually a monostable multivibrator, which is triggered by the coincidence of the analog voltage to be stored with a sawtooth ramp voltage. Regeneration and synchronization are provided by means of a pulse train (CLOCK) having about one hundred times the frequency of the ramp voltage. The period of the: monostablie must. be approximately equal to that of the ramp - to within one CLOCK pulse time. Read-out is by: the conventional sample-and-hold technique. Stability is excellent and; as will be seen. from the description to follow, the fractional accuracy is equal to the ratio of CLOCK pulse time to ramp time.

\subsection{6 .02 .2 Operation of PHASTOR}

The block diagram and typical waveforms of the system are shown respectively in Figures 1 and 2. The RAMP CYCLE (Figure 2) is started by the turn on of TRIGFF (Figure 1) and turned off by the schmitt trigger detector. The purpose of the HOLD OFF. monostable is to allow some dead time between the finish of one ramp and the start of the next. As can be: seen from Figure 1, these processes occur. continuously and are precisely synchronized wi.th CLOCK. 
Writing is effeałed by a Comparator circuit, $\mathrm{C}$, to register coincidence between INPUT and RAMP, and two control flipflops, C2 and C3. (The states of these flipflops will be denoted by $\mathrm{C} 2 \cdot \mathrm{C} 3, \overline{\mathrm{C} 2} \cdot \mathrm{C} 3$, etc..). In the present model writing is done manually by closing the switch: INITIATE STORE. This is effective - only if $\mathrm{C2} \cdot \mathrm{C} 3=1$. Then, the ensuing sequence of events, shown in Figure. 2, is as follows:

(a) $\overline{\mathrm{C} 2}: \mathrm{C} 3$ : await start of next ramp cycle, continue to regenerate previously stored information;

(b) $\mathrm{c} 2 \cdot \overline{\mathrm{C} 3}$ : clear storage element, await start of next ramp cycle;

(c) $\overline{\mathrm{C} 2} \cdot \overline{\mathrm{C} 3}$ : gate Comparator output pulse to storage element;

(d) C2.C3; operation complete, enable regeneration, ready for next INITIATE STORE.

Operations (b), (c), and (d) occur at successive turningson of TRIGFF. Figure 1 also shows how CLOCK is used to time the. writing and regeneration processes.

The sample-and-hold read-out operates continuously in the present system, since there is but a single storage element. It contains a flipflop (circuit, Figure 5) which is set by TRIGFF, allowing RAMP to charge-up a capacitor. The charging ceases. when the flipflop is reset by the pulse (O/P) from the storage: unit.

\subsubsection{Brief Description of Circuits}

Figure 3 shows the circuits appropriate to the upper left-hand section of Figure 1 . The RAMP GENERATOR is of the bootstrap type, capacitor $\mathrm{C}-1$ charging at a uniform rate after transistor T-2 is turned off by the positive excursion of TRIGFF. Detecting the top of the ramp is done by the SCHMITT TRIGGER, whose output, gated with CLOCK, turns on the HOLD OFF monostable. This immediately resets TRIGFF, with the signal CLAMP, thereby shutting off the ramp. HOLD OFF eventually returns to its 
quiescent state, the next clock pulse then turns TRIGFF back on and a new cycle begins..

The storage unit together with its input gates and regenerative loop (via transistor $\mathrm{T}-3$ ) is shown in Figure 4. The time constant of the circuit is adjustable by means of the potentiometer $R-3$. It should be noted that precise adjustment is not necessary. Since writing and regeneration are controlled by CLOCK, the time constant need be equal to the RAMP CYCLE time to within only one CLOCK perïod.

The sample-and-hold circuit of Figure 5 has already been mentioned in the previous section and needs no further description. Figure 6 shows the basic configuration of the PHASTOR flipflops.

\subsubsection{Further Development}

This version of PHASTOR stores only one analog signal, but the extension to several storage units is expected to be straightforward. A simple digital addressing system will at a later date be added to the system to control both the writing into the various storage units and the selection of their outputs into the sample-and-holdicircuitry o) ; 


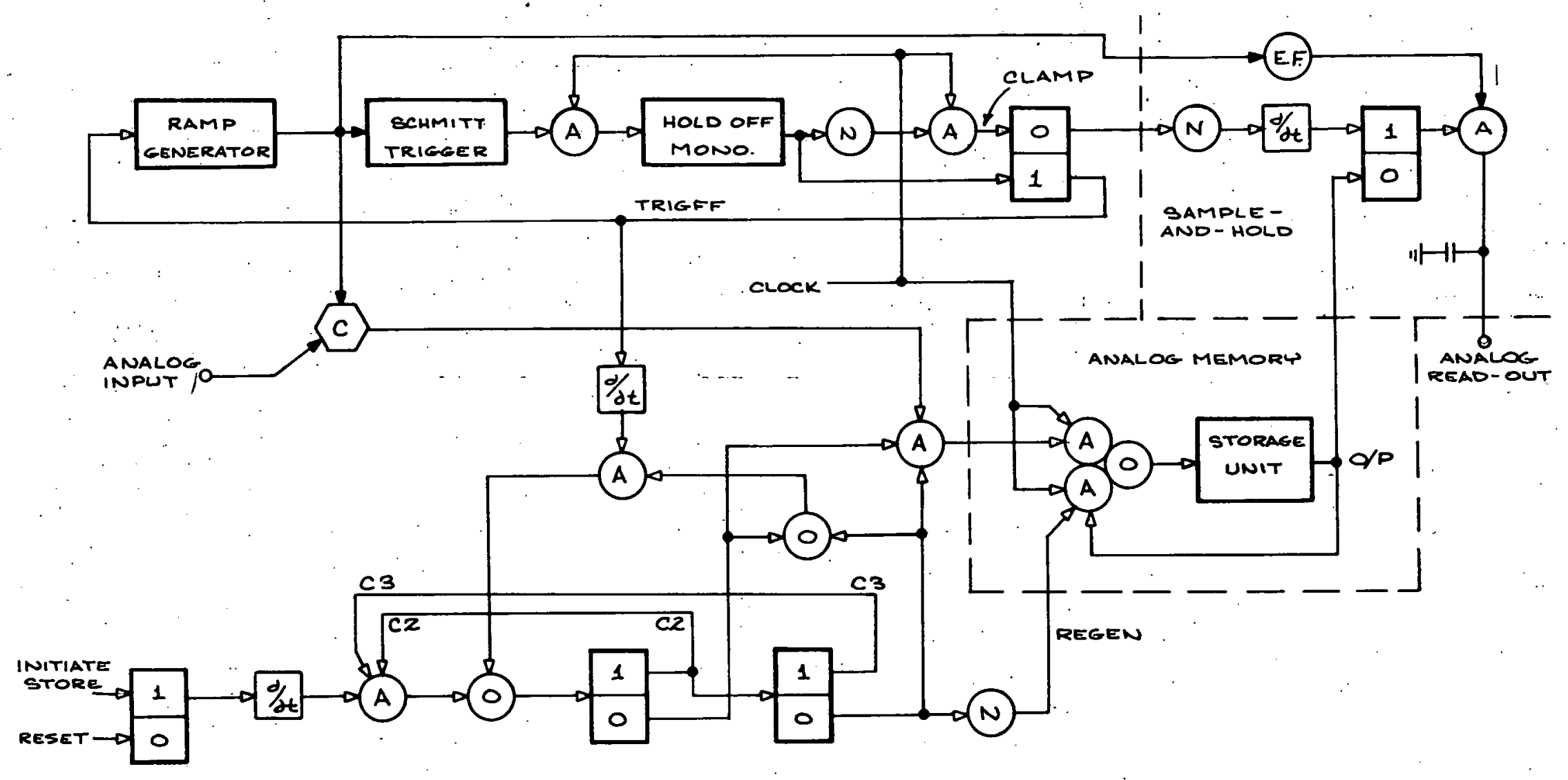

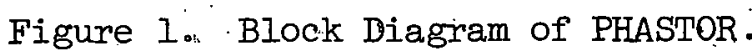




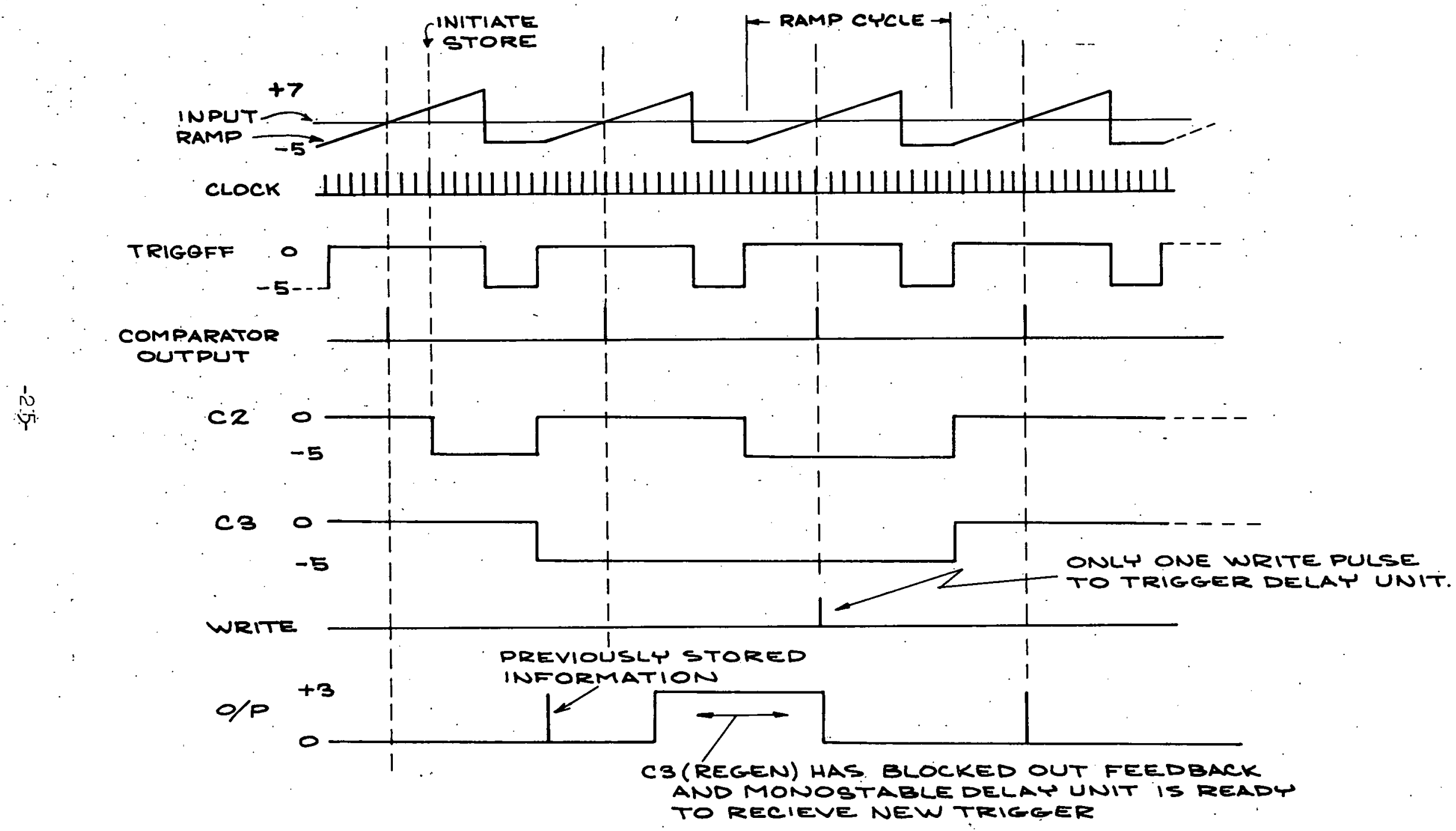

Figure 2. PHASTOR Wavéforms . 


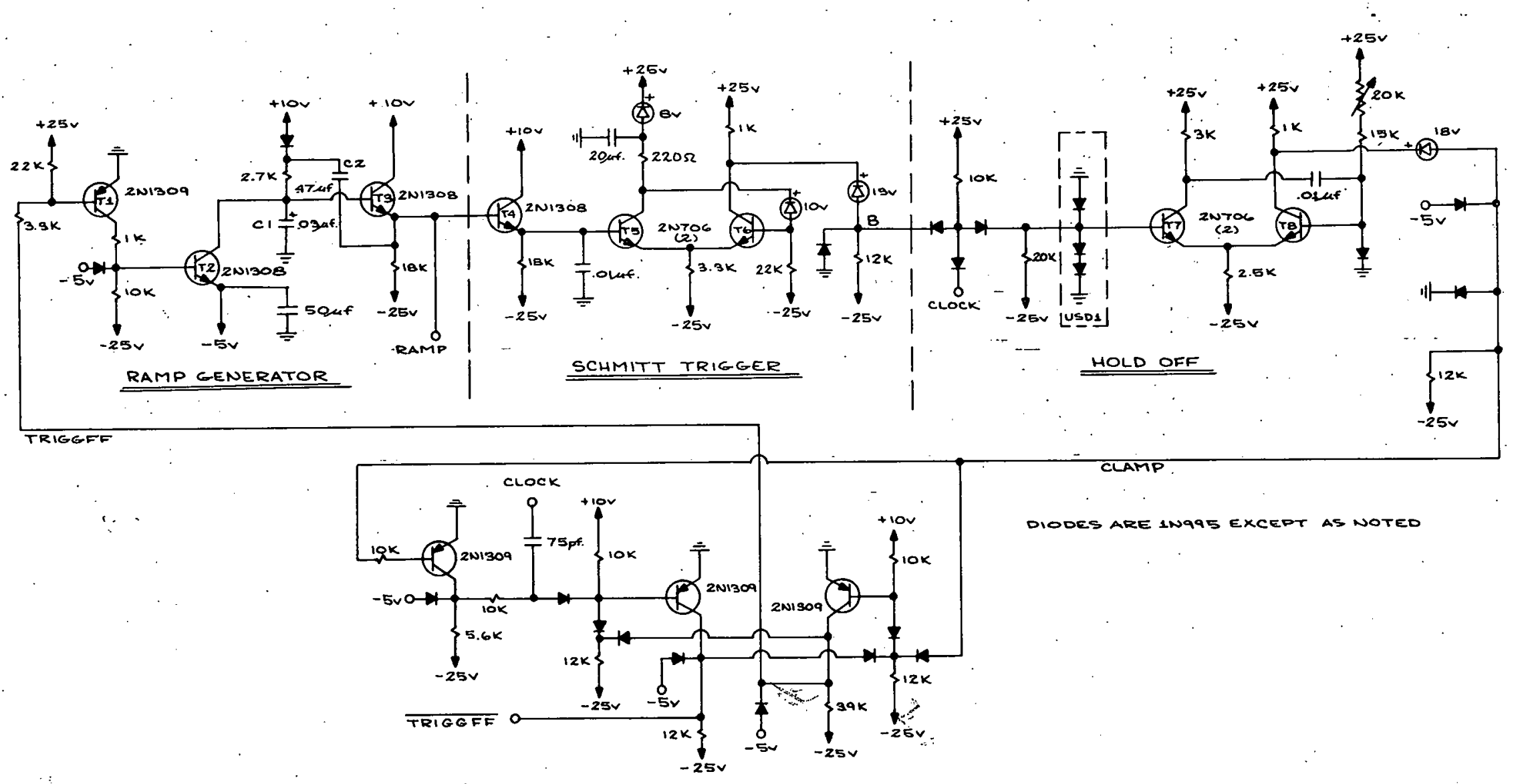

Figure 3. Waveform Generator Circuitry. 


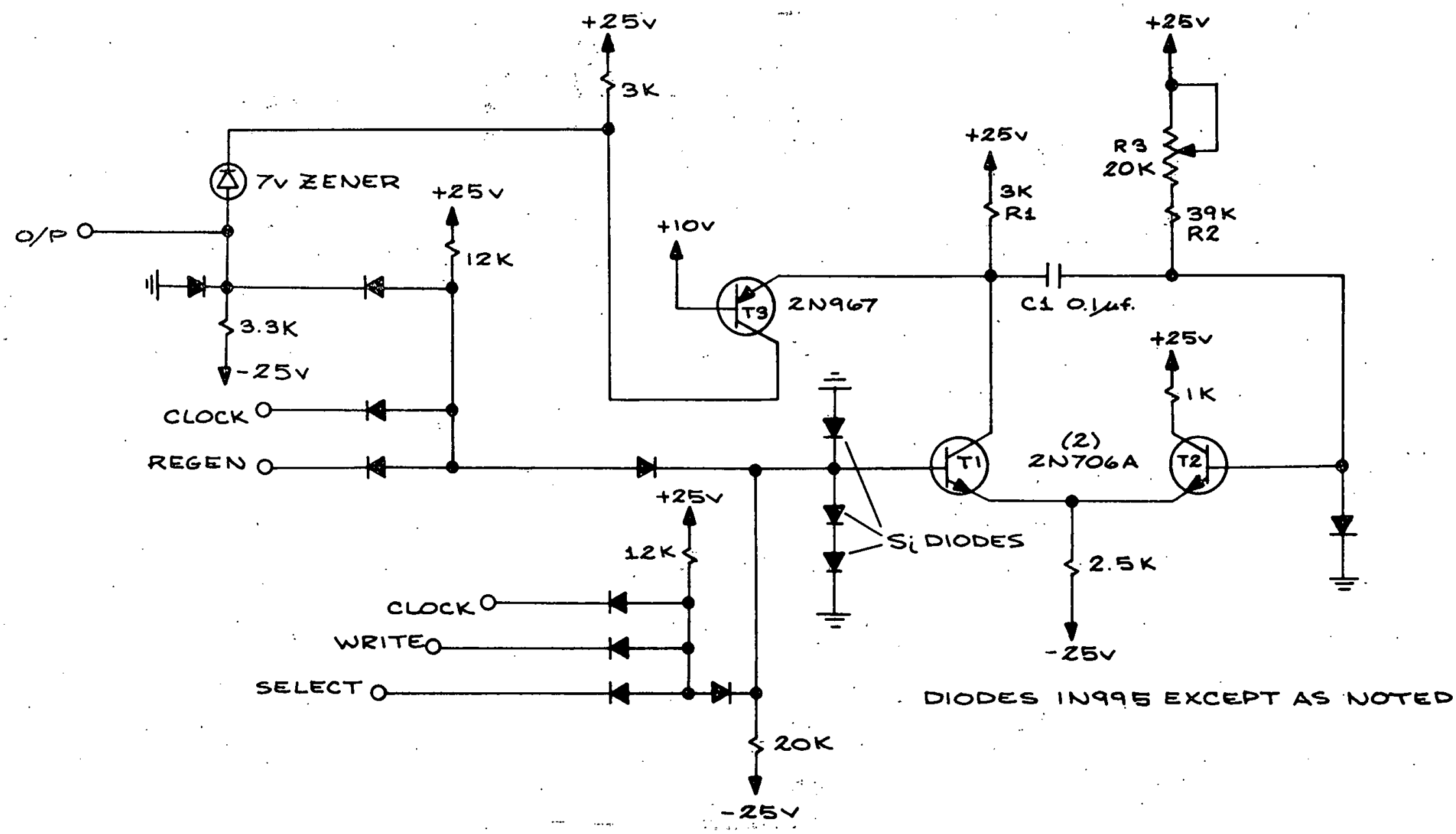

Figure 4. Monostable Multivibrator Storage Unit. 

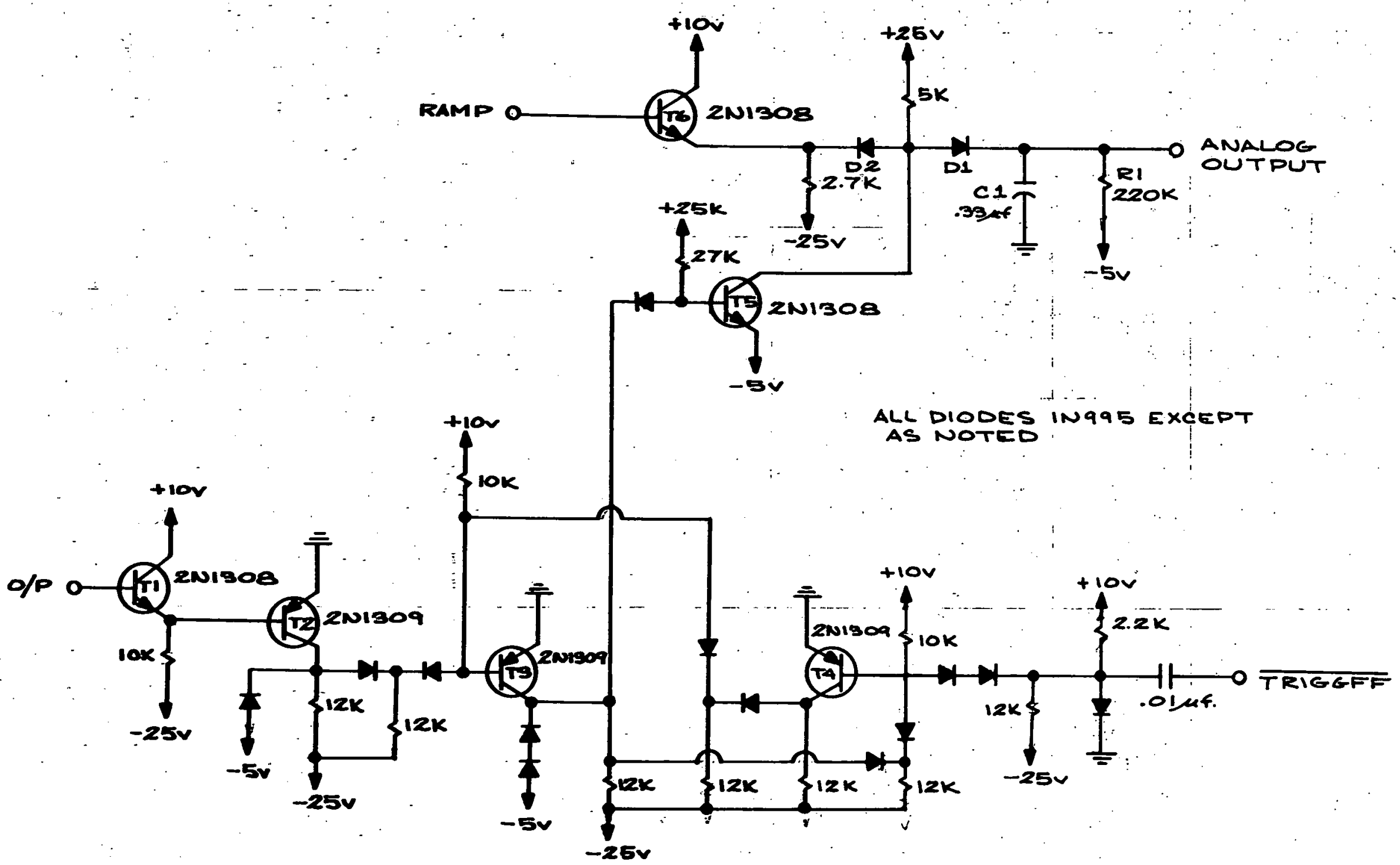

Figure 5. Sample and Hold. 
is
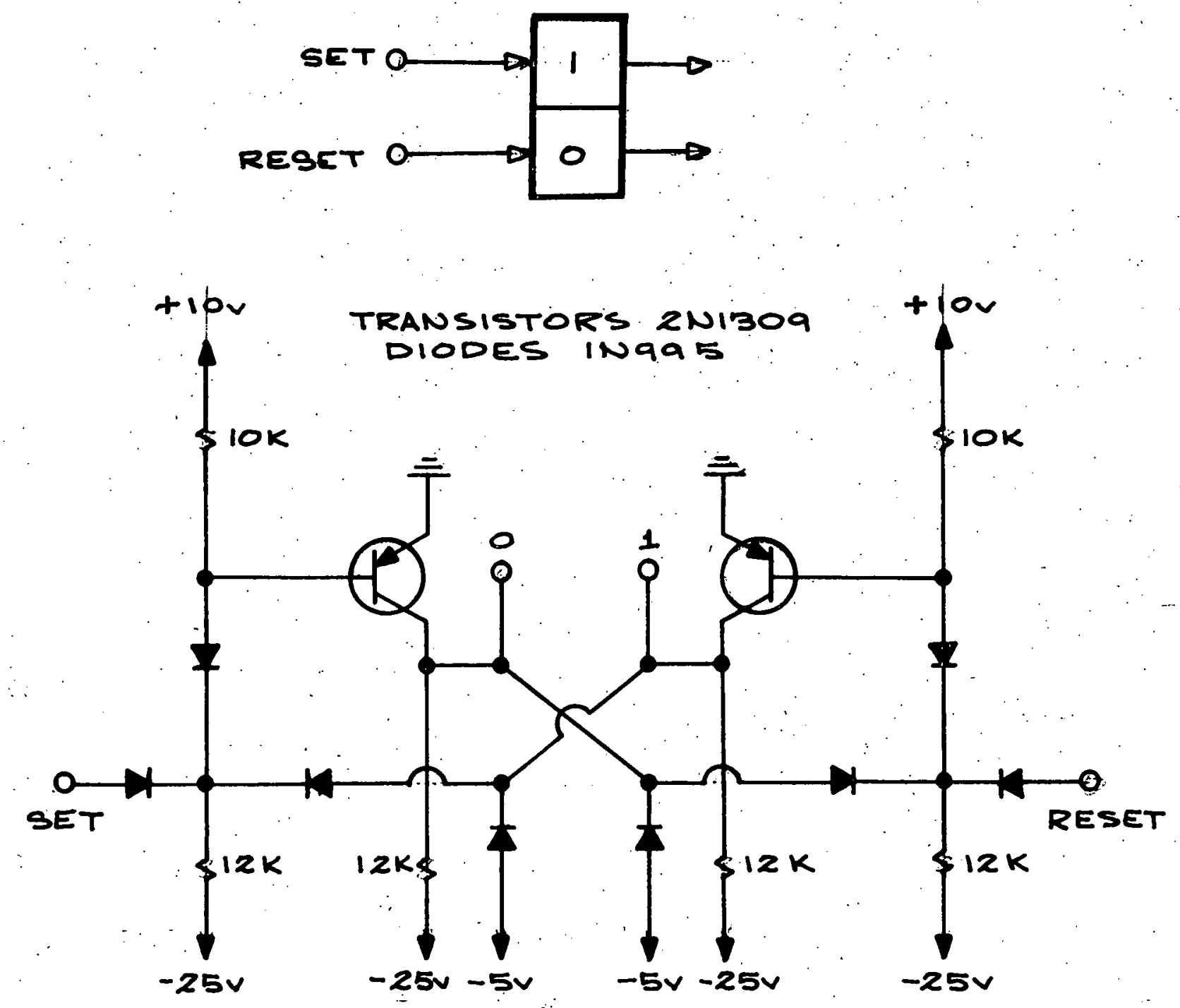

Figure 6. PHASTOR Flipflop Circuit

$-29-$ 
2. HARDWARE SYSTEMS RESEARCH

(Supported in part by the Atomic Energy Commission under Contract No. $\operatorname{AT}(11-1)-1469$.

Summary

The Artrix System has been built. The present report by John Esch, Bill-Kubitz; Pete Oberbeck and Dave Rollenhagen is devoted mainly to problems of check-outi...

In the Pattern. Transform Analysis work David Casasent outlines a proposal for an on-line Fourier transform system.

\subsection{The Artrix. System}

\subsection{0 .811 .1 Construction}

The construction of Artrix has been completed and most of the system.has been commissiened. It was necessary to install bellows between the cameras and their corresponding storage tubes (Memotrons) to shield against dust and ambient light.

\subsubsection{Pen Circuitry}

The design of the pen shaping circuit has been completed. It was determined that when a very narrow pen pulse was employed, the amount of charge placed on the storage surface of the Memotron was insufficlent to capse writing. This problem was solved by making the pen pulse longer and by stopping the deflection of the writing beam in the memory for the duration of the pulse. In this way enough charge 1s deposited on the storage surface to produce writing. In order to stop the deflection of the beam in the memory, 1t was necessary only to gate off the constant current drive in the horizontal. sweep generator with a two microsecond pulse. It is not necessary to gate the vertical sweep, since, during this short time, its motion is small enough to be neglected. 


\section{$2.10: 811.3$ Video Levels}

The video output level turned out to be lower than the 1.0 volt anticipated during design of the system. The nominal video output is about 0.25 :volts on a black level of 0.25 volts. This presents two problems to the present circuitry: 1) the circuits fail to make the distinction between the actual video and the DC black reference level; and 2) the total signal amplitude: is too small to cause the circuits to operate at all. To resolve this difficulty an additional circuit will be designed and used in front of the present circuits. This new circuit will have a threshold adjustment for eliminating the black level and will provide sufficient gain for the 0.25 volts of video remaining to produce a 1.0 volt pulse for the circuits following. With the adition of this circuit it should be possible to complete the check-out and the Artrix System will be operational.

\subsubsection{Processing Unit Logical Section}

Two features discussed in the last report, namely, synchronizing the horizontal and vertical master counters with . the display and storage system.'s deflections, and interlacing of lines, have been incorporated into the system and are operating perfectly. A third feature, designing for lines with negative slopes, is in the process of being tested.

Two new system problems arose when the sections of the Artrix System were connected together. The Processor sends. synchronizing signals to the camera control unit at a rate of $31.5 \mathrm{kHz}$. The camera control unit then generates horizontal sync pulses at a rate of $15.75 \mathrm{kHz}$ for the T.V. system. Since there are two synchronizing sigalls into the control unit for each horizontal sync pulse produced; it was necessary to design and install logic circuits that would insure that the horizontal sync pulse came at the correct input synchronizing signal. Before this correction was made it was possible for the $\mathrm{T} . \mathrm{V}$. system to be operating a half line off from the processor, depending on which phase the camera control unit slipped into when the power 
was turned on. As it now stands, the relative..phase of the Processor and camera control is monitored continuously and corrected automatically.

The second problem was that the T.V. system was tracking about one-twentieth of a horizontal line off from the Processor. This tracking problem was traced to the camera control unit whose horizontal-sync pulses are delayed about $3 \mu \mathrm{s}$ from the input synchronizing pulses. This was overcome by advancing the input synchronizing pulses by $3 \mu \mathrm{s}$. As a.result, the logical signal indicating all ones in the horizontal master counter now always occurs at the same point inside the horizontal sync pulse.

Another area of progress was that of ground noise. Even though the system was working it was felt that the noise level was too great. Consequently, power and ground busses were enlarged, thereby reducing the noise by about:2/3. However, there was still unexplained periodic noise which was at a frequency not produced by any of the Artrix circuitry : Eventually the power supplies themselves were isolated, revealing a fault in their desigh which was corrected.

\section{$2 \cdot 10.09 .5$. Line and Circle Generator}

- Line and circle. generation for the Artrix System is accomplished by means of Lissajous patterns. At present it is possible to draw lines and circles using sinwt, cosut, and -sinut. All parts of the Processor are working; a circle is drawn by indicating its center and a point on the circumference; a line is drawn by indicating its extremities.

For the circle generation it is necessary to let the radius of the circle expand until the indicated point on the circumference 1s reached. This is done by comparing the increasing sinusolds, sinut and cosut, with the vertical and horizontal position, respectIvely, of the point on the circumference. If both comparators show colncidence simultaneously, the desired radius is reached, and further increase is stopped. 
A schematic of the comparator is shown in Figure 1 . It will produce a $200 \mu \mathrm{s}$ pulse ( -5 volts to ground) if its inputs are within $50 \mathrm{~m}$ V of each other. The input range is \pm 15 volts. Matched diodes and transistors are used in the circuit to increase its accuracy.

All other circuitry (described in prewious reports) is functioning properly, indicating that on-line processing in a graphical display is possible through the use of hybrid-analog-digital circuitry. 


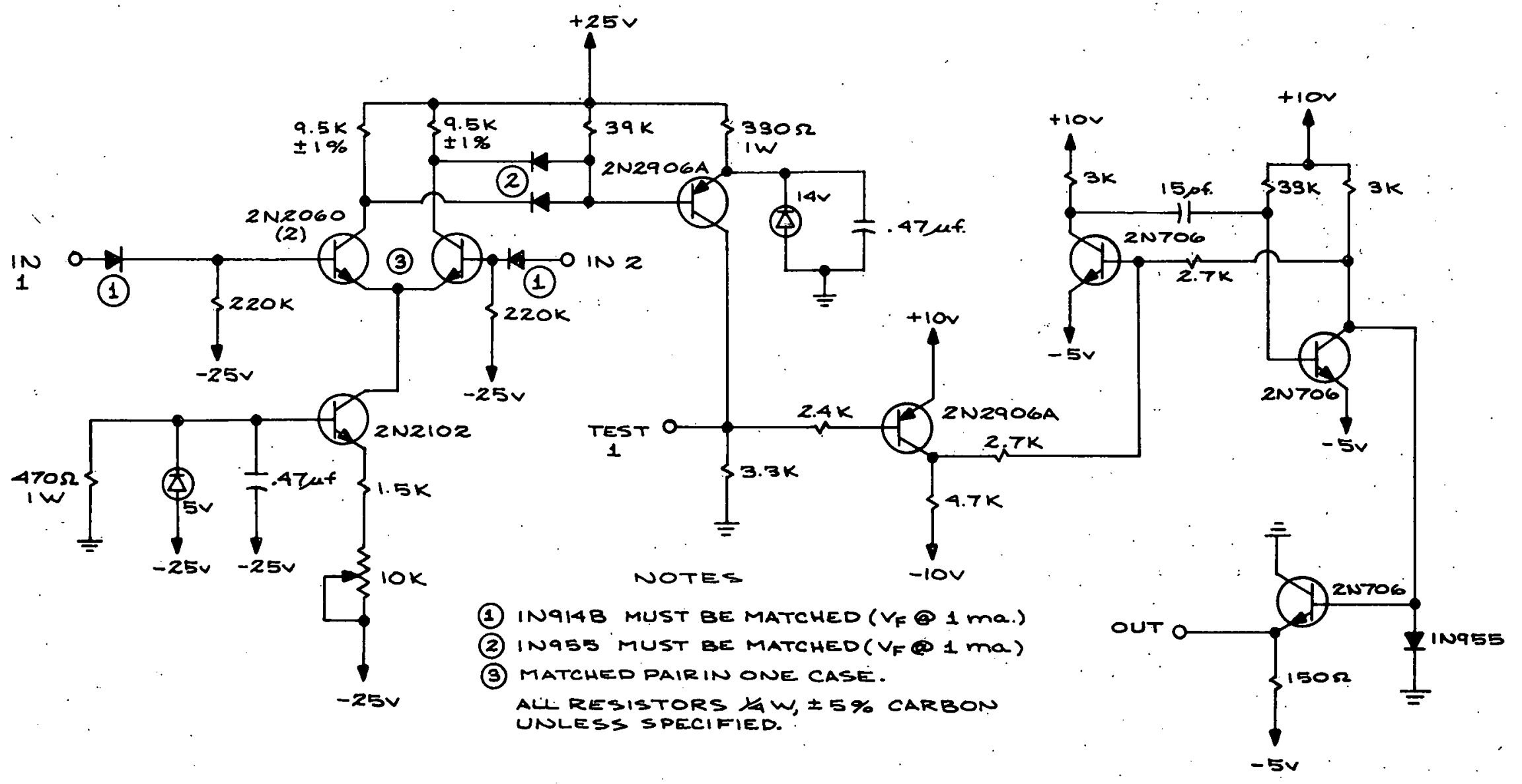

Figure 1. Comparator. 


\subsection{Pattern Transform Analysis}

\subsubsection{On-Line. Fourier Transform System}

Figure 1 is the block diagram of a proposed system for the display and analysis of the Fourier Transform of a pattern. This system is: unique in that it will allow on line operation with incoming video signals. The basic operation is to write one frame of video information onto a transparent medium and them flash through this transparency the light of a gas laser, picking up the Fourier transform of the image on a vidicon. The intermediate storage problem will be handled in an extremely elegant manner by means of the solid state light modulator pictured in Figure 3 and discussed in Section $2.12 .03 \cdot 3$.

The light source is a $50 \mathrm{mw}$ continuous wave gas laser capable of producing an extremely precise linearly polarized, uniphase, single mode wavefront at 6328 \&.

The light shutter consists of $4 \mathrm{KD}_{2} \mathrm{P}$ crystals (Potassium Dideuterium Phosphate) arranged optically in series and electrically in parallel capable of producing approximately a I ms pulse of light every $32 \mathrm{~ms}$.

The Collimator expands the laser beam to a diameter of approximately one inch while maintaining a plane wavefront within one-tenth of a wavelength.

The associated spatial filter blocks the passage of light not contained in the main laser mode and removes spatial noise from the wavefront. In this manner noise from internal scattering of the high gain laser oscillator and the scattering and diffraction of the coherent beam from surfaces and inhomogeneities in external components placed in the laser beam's path are eliminated, and a smooth gaussian shaped intensity profile in a large diameter collimated beam is produced.

The transparency plane contains a specially designed crystal exhibiting the electro-optic effect and an off axis electron gun. This effects a simultaneous area modulation of the incident beam with 
the electron gun being the control element. The operation of this modulator is explained in Section 2.12.03.3.

The Fourier transform of the image placed in the transparency plane is formed in the focal plane of lens 1 , the original image is reconstructed in the focal plane of lens 2 , and the Fourier transform is again constructed in the focal plane of lens 3 .

The first Fourier transform plane is an excellent one in which to mask the pattern and the: modulator induced DC level; otherwise this must be done on the vidicon faceplate itself to reduce the dynamic response requirements of the vidicon. A spatial filter can also be located in this first Fourier transform plane. Lens 3 will not be necessary if only the filtered reconstructed image is desired. In this case the image can be picked up in the focal plane of lens 2 by a vidicon.

\subsubsection{Fourier Transform Optics}

The fundamental principles of using lenses to obtain the Fourier transform of planar density pätterns in a coherently illuminated optical system will now be reviewed.

Figure 2 shows the transparency plane, lens 1 , and the first Fourier transform plane of Figure 1 . The transparency or object plane is the front focal plane of the liens and the first Fourier transform plane is the image or back focal plane of the lens.

The incident electromagnetic wave is assumed here to be a. planar, unpolarized, unmodulated, monochromatic wave of uniform spatial amplitude propagating in the $\mathrm{z}$ direction and described by

$$
E_{\text {inc }}=E_{O} \exp [j \omega t]
$$

where $\mathrm{E}_{O}$ is the maximum amplitude of the electromagnetic vection and $\omega$ is the radian frequency of the electromagnetic radiation. The front focal plane is assumed to contain a light modulator characterized by

$$
f(x, y)=f_{0}(x, y) \exp \left[j \Phi_{0}(x, y)\right]
$$


The wave emerging from this input plane is thus diffracted and can be described by

$$
E_{0} f_{0}(x, y) \exp j\left[\omega t+\Phi_{0}(x, y)\right]
$$

A reference plane is established through the origin of the input plane and perpendicular to $\overrightarrow{\mathrm{k}}$ and described by

$$
a_{1} x+a_{2} y+a_{3} z=0
$$

where the coefficients are the direction cosines of $\vec{k}$. They can be evaluated by recalling that all light traveling in the $\vec{k}$ direction at the left of the lens will be summed at some point $\left(x^{\prime}, y^{\prime}\right)$ in the back focal plane and further that light in the $\overrightarrow{\mathrm{k}}$ direction passing through the center of an ideal lens will not be diverted. Using these facts and letting $R$ be the distance from the center of the lens to the summing point, $F$ the focal length of the lens, the coefficients are

$$
\begin{aligned}
& a_{1}=\frac{x^{\prime}}{R} \\
& a_{2}=\frac{y^{\prime}}{R} \\
& a_{3}=\frac{T^{2}}{R}
\end{aligned}
$$

Now the phase at all polnts can be determined. At the reference plane:

$$
\Phi_{\mathrm{R}}=\Phi_{0}(\mathrm{x}, \mathrm{y})^{\cdots}+\frac{2 \pi}{\grave{\lambda}} \dot{\alpha}
$$


where $d$ is the perpendicular distance from a point in the front focal plane to the reference plane.

$$
d=a_{1} x+a_{2} y
$$

and $\lambda$ is the electromagnetic. wavelength. At the summing point

$$
\Phi_{S}=\Phi_{0}(x, y)+\frac{2 \pi}{\lambda}(d+L)
$$

where $L$ is the optical path length from the origin of the input plane to the summing point in the image plane. The beam issuing from a section dxdy contributes an amplitude:

$$
E_{0} \exp (j \omega t) f_{0}(x, y) \exp \left[j \Phi_{S}\right] d x d y
$$

and the total electric field at the summing point is from equations (7), (8), and (9):

$$
\begin{gathered}
E_{S}=E_{O} \exp (j \omega t) \iint f_{0}(x, y) x \\
\exp \cdot\left[\Phi_{0}(x, y)+\frac{2 \pi a}{\lambda F}\left(x^{\prime} x+y^{\prime} y\right)+\frac{2 \pi L\left(x^{\prime}, y^{\prime}\right)}{\lambda}\right] d x d y
\end{gathered}
$$

By defining $\frac{2 \pi}{\lambda F} \quad x^{\prime}=w_{x}$ and $\frac{2 \pi}{\lambda F} y^{\prime}=w_{y}$ as the spatial frequencies, recalling that $L$ is a constant and thus contributes only a constant phase shift, replacing $E_{i n c}$ by its RMS value $\bar{E}$ which assumes integration over many cycles at the electromagnetic frequency, and letting $a_{3}=1$ which assumes small diffraction angles, equation (10) becomes

$$
E_{s}=\bar{E} \iint \bar{f}(x, y) \operatorname{expj}\left[w_{x} x+w_{y} y\right] d x d y
$$


which is the two-dimensional Fourier transform of the field function in the front focal plane. . High-precision optics and strong coherent sources have: made such a system highly practical and it is now entirely possible to process up to $10^{6}$ bits of positional information in parallel.

\subsection{2 .03 .3 Transparency Plane}

One of the unique aspects of this system is the solid state: light modulator which is placed in the transparency plane of Figure 1 . The principles of operation have been known for nearly 100 years. The first working models of such a device were invented in 1934 in Hungary by C.F.Pulvari for use as a sound recording device and in 1938 in Germany by M. Von Ardenne for facsimile transmission.

The proposed model for this application is shown in Figure 3 . It. makes use of the electro-optic and Pockels effects in a transmission instead of a reflex system.

The electro-optic effect in a crystal is a change in its optical properties which occurs in the presence of an electric field. The Pockels effect occurs when the electric field is produced in the same direction as the incident wave coincident with the optic axis of the crystal. (The Pockels and Kerr effects have been widely used in the past to achieve a retardation variable with voltage.) Crystals of the $\overline{4} 2 \mathrm{~m}$ class such as Potassium Dihydrogen Phosphate (KDP), Potassium Dideuterlum. Phosphate $\left(\mathrm{KD}_{2} \mathrm{P}\right)$ and Ammonium Dihÿdrogen Phosphate (ADP), exhibit a strong electro-optic effect, and, of these, KDP appears to be best due to present technological abilities. A Z-O cut electro-optic crystal, that is, one in which its principle faces are perpendicular to the optic axis, is necessary. The $z$ axis is the optic axis and the direction of light propagation. The sides of the crystal are oriented parallel to the $x$ and $y$ axes and the entire crystal is then placed between two polarizers. In actual use the input wave will be Iinearly polarized but, for ease of discussion, unpolarized incident light will be assumed. The two polarizers have their polarizing axes oriented at $90^{\circ}$ (see Figure 3). A wave inc1dent on such an arrangement will be blocked since the polarizing axes 
are orthogonal. However, an electric potential applied across this crystal causes it to become biaxial and the plane polarized light incident on the crystal separates into an ordinary ray and an extraordinary ray. These rays are perpendicularly polarized. The ordinary ray vibrates perpendicular to the optic axis and travels at constant speed while the extraordinary ray vibrates parallel to the optic axis and travels with a velocity dependent on the applied voltage. The resulting ray is thus elliptically polarized. The light pattern which is transmitted.will correspond to the charge pattern placed on the crystal surface by the modulated electron beam from the off axis electron gun.

The transparent conductive coating on the back of the KDP crystal establishes a continuous reference plane for the electric field produced across the crystal due to the electrons deposited by the gun. By holding the anode of the CRT and the conductive electrode at a potential such that the secondary emission ratio of $\mathrm{KDP}$ is less than 1 , there will be more electrons produced by the scanning beam than by secondary emission and the surface will become negatively charged producing a: field across the crystal. A spray of electrons from the gun and secondary emission greater than. I can then be used to clear the crystal face for the writing of the next frame of information. In this manner a frame rate of 30 frames..per second and a resolution of greater than 200 lines can conceivably be achieved. 


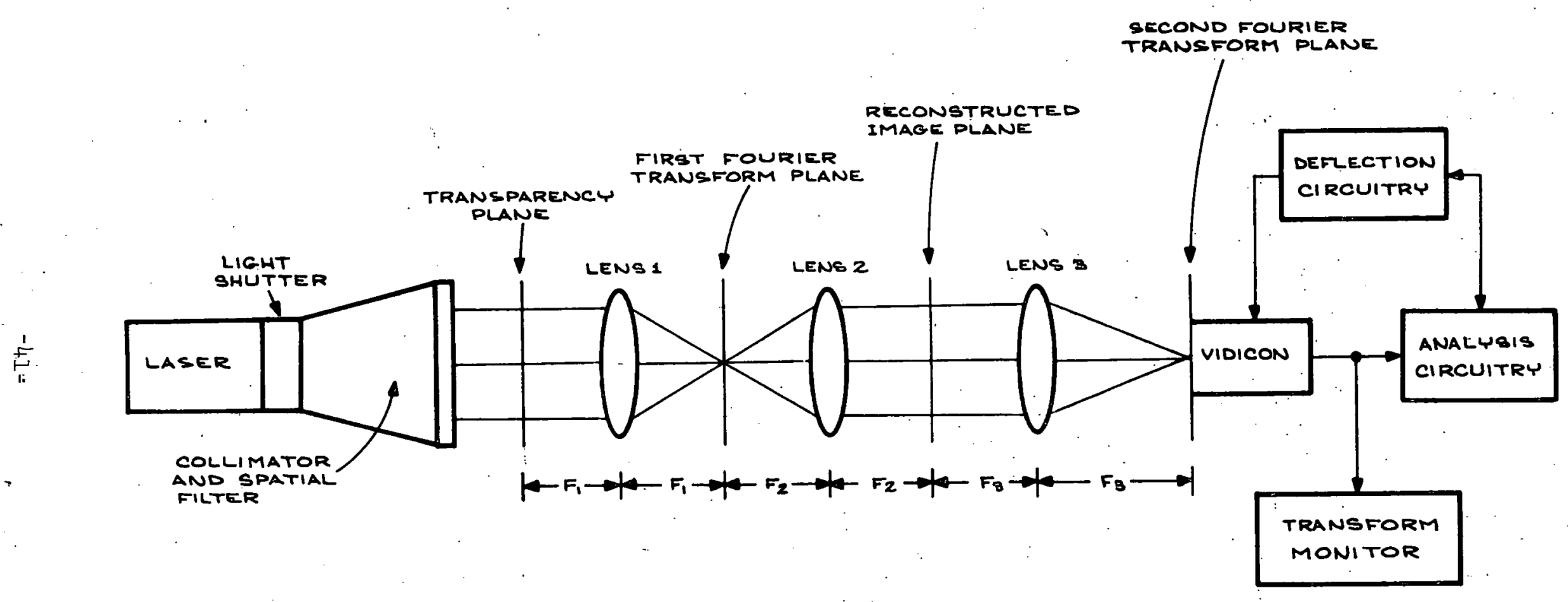

Figure 1. On-Line Fourier Transform System. 


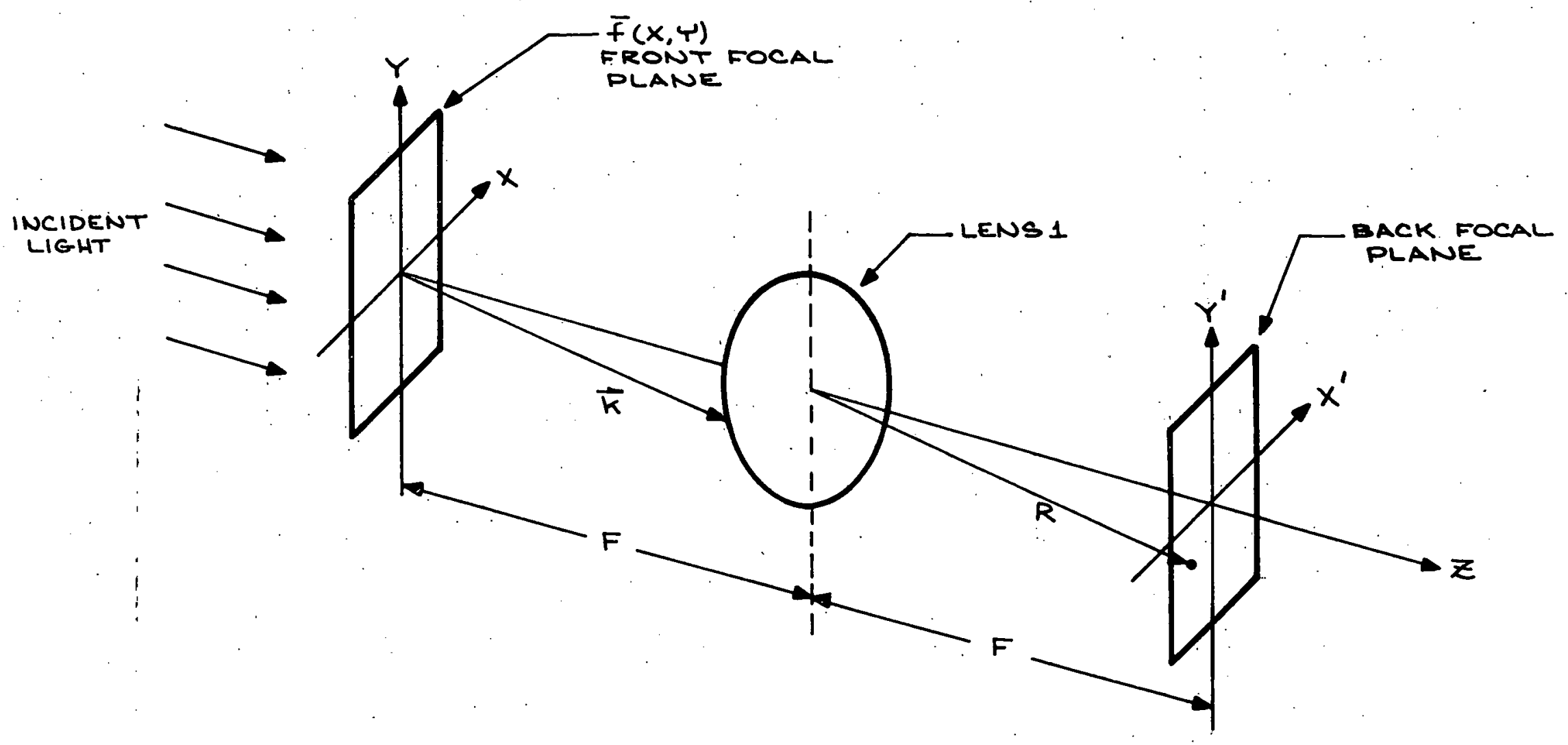

Figure 2. Fourier Transform Optics. 


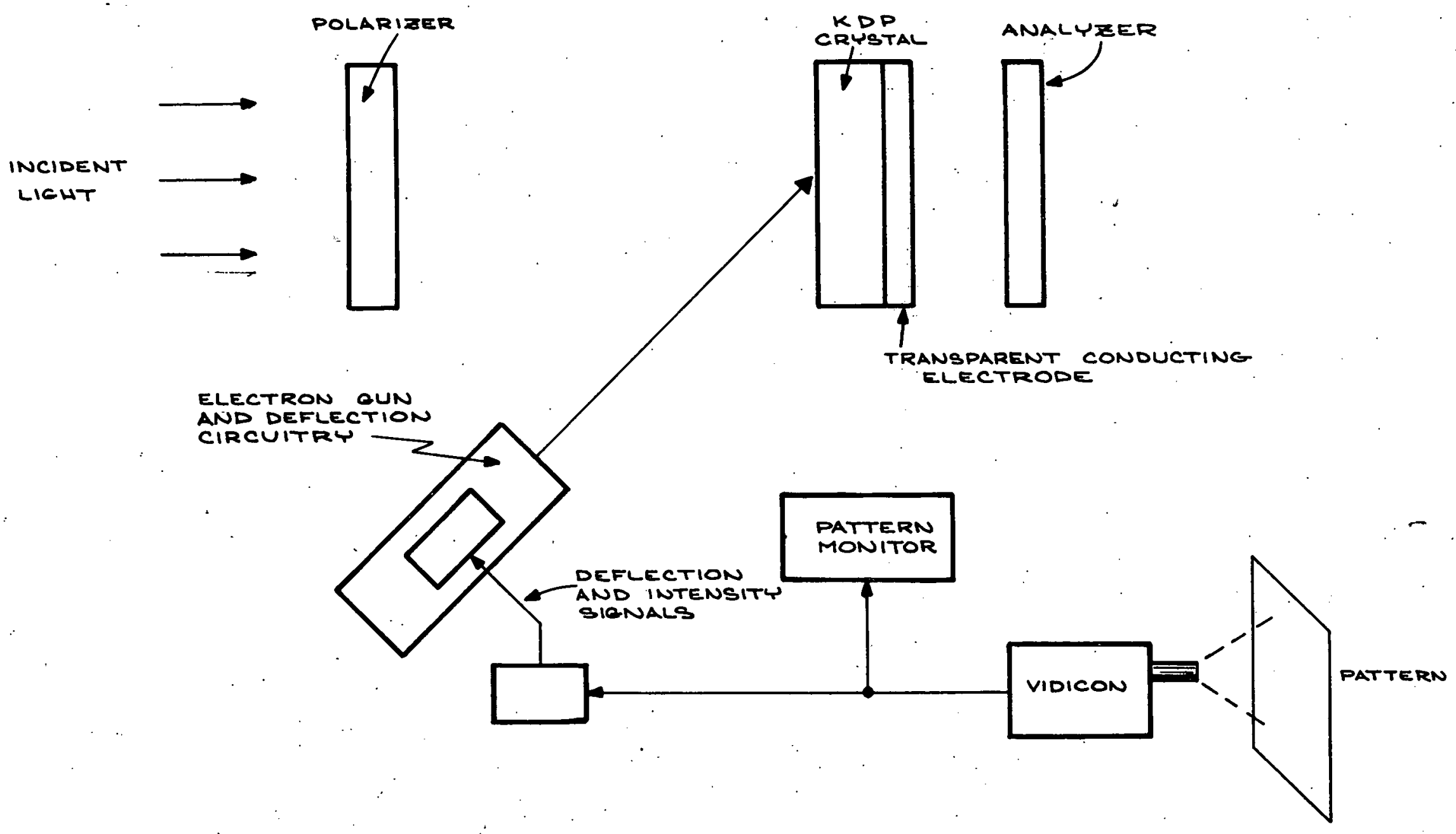

Figure 3. Solid State Light Modulator. 


\section{SOFTWARE AND TIME-SHARING SYSTEMS RESEARCH}

(This work is supported by Contract No. AT(11-1)-1469 of the Atomic Energy Commission and in part by the University of Illinois.)

This section provides a description of the work in progress on the Illinois Time-Sharing System which is currently being implemented on the ILLIAC II/PDP-7/IBM. 1401 hardware.

Briefly the progress made this quarter includes the development of an improved version of $B \phi \phi \mathrm{TS}$, the planning and coding of Input-output routines for $B \phi \phi \mathrm{TS}$, the programming of certain executive routines for the new time-sharing system, the completion of SYSTIM, SYSTAP, and STACK for use as system subroutines under time-sharing, the improving of SYSFBN and S $\phi R T-$ MERGE, the debugging of the Binary Card Loader and SYSDSK (a highly complex Disk-Drum System Routine); the arrival of new versions of the disk dump and reload routines, the writing and debugging of a. TRACE routine for B $\phi \phi T S$ ' programs, the further specification and coding of the PDP-7 interfaces and

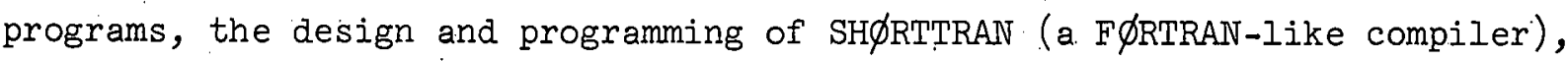
the preliminary specifications for the graphical display console and the internal construction of the related software, and the design of further time-sharing equipment interfaces as well as the programming of test routines for the PDP- 7 and of a new disk bootstrap program.

A Programming Memorandum (No. 50, May 11, 1.966) describing the design of TIPSY and giving examples of its use was distributed. Weekly meetings are continuing for all system programmers involved in the development of the time-sharing system.

The sections of Part 3 are the responsibility of the following

peopie:

Greninger, L. - B $\phi \phi T S$ and SYSFBN and FRDSK

Fisher, R. - Executive Programs for Time-Sharing

Neuhaus, P. - System Subroutines for Time-Sharing

Ellis, C. - Sort-Merge

Berg, M. A. - Binary Card Loader

Nuspl, S. - SYSDSK, the Disk-Drum Routine, and TRACE routine under BOOTS

Lyon, R. - Garbage Collection

Otis, A., Koopman, L. - PDP-7 Programming 


$$
\begin{aligned}
& \text { Bennett, D. - SHфRTTRAN } \\
& \text { Richardson, F。 - Graphical Display } \\
& \text { Shepard, C. D. - Miscellaneous } \\
& \text { Willard, R., Nuspl, S. J. - Hardware System } \\
& \text { C. W. Gear, Prịcipal Investigator }
\end{aligned}
$$

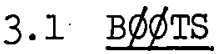

\subsubsection{General}

Progress this period has consisted of trying to get a working copy of the new 'B $\phi \phi \mathrm{TS}$ ', (the time-share command processor). All code checking was done from a console using the present time-sharing system. The facilities for running programs have not yet been checked but since they have been taken directly from the current $B \phi \phi \mathrm{TS}$, it is hoped that they will work cor:rectly. Several new commands have been added to the new version of the processor. Namely, the following will be available:

(a) the facility to change the read-only dictionary from the system's dictionary. to any user's dictionary thus permitting a crude form of common files and file pools;

(b) the line numbering capability;

(c) permitting all file references to be done by explicit references to file's line numbers as opposed to the present 'pointer' system.

The full Sort-Merge is not yet implemented, rather a stop-gap merge with no sort is filling in until the full package is ready.

Presently attempts are being made to.fit the new version of $B \phi \phi T S$ into the system environment in which it is eventually to run.

\subsubsection{Input-Output Routines}

The input routine for the new $\mathrm{B} \phi \phi \mathrm{TS}$ is written but not yet debugged. This routine will take submitted decks and enter them in a particular user's file as designated on the ID card.

An output routine for $B \phi \phi \mathrm{TS}$ is being planned. It should take all files that have been. "outputed" for the consoles and write them onto a tape for later printing. 


\subsection{Executive Programs for Time-Sharing}

The basic monitor package for the new time-sharing system has been designed, flowcharted, programmed and debugged. Specifications for the ILLIAC II end of the PDP-7 communications are complete. C $\varnothing \mathrm{MPR} \phi$, the Console Command Processor, will consist of three fairly independent programs-C $\varnothing \mathrm{MPR} \varnothing \mathrm{A}, C \varnothing \mathrm{MPR} \varnothing \mathrm{B}, \mathrm{C} \phi \mathrm{MPR} \varnothing \mathrm{C}$. A will reside in high core, B will be brought into core when needed by $A$, and $C$ will be brought in to help $B$ when necessary. Since consoles have priority and since a console which is interacting has priority over one which is in execution, $C \phi M P R \phi$ is neither schedulable. nor swappable.

C $\varnothing$ MPR $\varnothing$ A will run the SPU (Satellite Processor Unit) channel under interrupt control and will continue to process lines regardless of what else is in core. It has been flowcharted and programming has begun.

$C \phi \operatorname{MPR} \phi \mathrm{C}$, the command language processor and general supervisor of all consoles, has been flowcharted. It interprets and handles "short" commands and sets up taisk lists for the scheduler on "Iong" commands. (Short = PRINT, SAVE, LOAD, DEFINE, etc. Long = S $\phi R T$, MERGE, RUN, SEARCH, etc。)

At present, the current ILIIAC monitor is being converted to use the PDP-7 for console communication rather than the MSR's. A subset of the PDP-7 package has been flowcharted, coded, and is now being checked out.

A reference for this subsection is: Programming Memorandum No. 42 , "COMPRO", November 23, 1965.

\subsection{System Subroutines for Time-Sharing}

The following programs for the new time-sharing system have been completed:

(a) SYSTAP which does all protected orders to the tape channels and is to reside in protected core.

(b) TSSAUX which uses SYSTAP and is used by TSYSI $\varnothing$ for tape inputoutput. This routine does tape manipulation and is to reside in the user core area.

(c) SYSTIM which takes care of all interval timer manipulation and resides in protected memory. Its functions fall into two main categories - CALL'S which are concerned with the clock itself (either setting the clock to interrupt or reading the 
clock), and CALL'S which are concerned with a certain number of "cells" used to accumulate time for different purposes.

(d) STACK, USTACK, USTAK3: which handles fast register 'saves' and 'restores' for the monitor. This resides in protected memory and enables a monitor subroutine to "CALL" itself.

Pertinent publications for the quarter were Programming Description 2, "SYSTIM" (April 29, 1966) and Programming Description 3, "STACK, USTACK, USTAK3!". (May 20, 1966).

\subsection{SYSFBN and FRDSK}

SYSFBN and FRDSK comprise the record-oriented disk input/output package for the ILIIAC II time-sharing system. Similar in action to the tape oriented SYSI $\varnothing$, SYSFBN inputs and outputs logical records from and to files of 'records located on the disk. FRDSK handles the track-by-track allocation of the available disk storage. Together, they form the user's window on the file-by-name system.

SYSFBN employs a system of correspondence numbers to permit the accessing of multiple input/output files by a user. Each file has, declared with it, a correspondence number and each SYSFBN "CALL" is executed on that file whose correspondence number is given in the "CALL".

As the final appearance of the time-sharing system becomes clearer, it is becoming more obvious what the desirable options of SYSFBN should be. Thus modifications continue. Attempts are being made at incorporating SYSFBN into the present system in order to gain performance data. Inefficiencies are already being corrected.

An unnumbered memorandum was distributed to the system programmers early in April describing the purpose options, calling procedures and parameters for SYSFBN and FRDSK.

\subsection{SORT-MERGE}

Progress has continued on the SORT-MERGE routines. A version has been completely programmed and the routine to be incorporated into the final. system is now planned and being coded.

$$
-47-
$$




\subsection{Binary Card Loader}

A Loader has been adapted for the time-sharing system which allows for two binary card formats. The disk file is used for intermediate storage by the Loader and for the user's program if that program is greater than $2 \mathrm{~K}$ words. A user's program that is less than $2 \mathrm{~K}$ words is stored in core. This cuts down on the run-time for small programs (the majority of runs).

The Scheduler calls the Loader to perform its job and transmits addresses which allow the Loader to do dictionary searches for the programs requested. Upon finishing its job the Loader sends back to the Scheduler the program 'start' address and the total length of the loaded program.

The Binary Loader is now being debugged under the present timesharing system.

\subsection{SYSDSK, the Disk-Drum Routine}

SYSDSK, the ILLIAC II system routine for handling all block transfers of data to and from the disk file with the drum used as a buffer, is completely planned and programmed. The debugging is at an advanced stage; the main problems are in the area of interaction with the interrupt monitor and emergency procedures in case of an error on the disk or drum. A detailed description of the parameters for SYSDSK and the possible operation codes is available and a description of the mechanisms in SYSDSK is in preparation. A brief exposition of the basic principles behind. SYSDSK follows:

The main operations in SYSDSK are the reading and writing of blocks of data 256 words long. The program initiating the 'CALL' to SYSDSK need only specify the core block and the disk track involved; the use of the drum as a buffer is automatic and based on a priority scheme which keeps copies of the most active disk tracks on the drum. The calling program may aid in the optimization of the drum by issuing preparatory commands and giving information as to whether a disk track will be needed again. However, this optimization information is not a prerequisite for a transfer and, in fact, the preparatory calls are ignored if the drum is too busy to make use of them. The calling routine is guaranteed that a block will be transferred when it gives a 'read', the transfer being rapid if the block is on the drum and possibly quite slow if the disk heads must be moved and a direct disk to core transfer initiated. In most cases, a block being written on the disk will first be written on the drum where it waits in a queue before finally being 
transferred to the disk.

The 256 possible drum blocks are divided into two areas--one reserved only for single block transfers and the other for "8 biock" trarsfers. A drum table of 128 words contains all the information required on the state of the drum block, the disk track number whose image is on the block, and a link making, the transfer a part of one of 10 queues which are used for optimization. In the case of the single block area there is one word in the table for each drum block; for the 8 block area only one word specifies the 8 block image of some set of 8 contiguous disk tracks. There are 16 "states" in which a drum block may be, each representing a different stage of preparatior: or progress in the buffered transfer. For example, when a "write" CALI is made, a free or low priority drum block is assigned to the track specified and the block is put into the drum queue where it competes with other core blocks for transference onto the drum. When the block is accepted as the next drum transfer, a different state is entered. Upon completion of the core to drum transfer, the CALL is successfully terminated as far as the program calling SYSDSK is concerned: However, another drum table state is entered--this being one in which the block is competing to be transferred onto disk. Upon being chosen as the next drum to disk transfer, the disk cycle is begun. This cycle successively initiates and waits for the seek, waits for and assigns a free core block as buffer, initiates the drum to core read, and finally starts the core to disk write. Adequate interlocks are provided so that no call to SYSDSK which might disrupt a sequence of states can produce a loss of data or the acquisition of erroneous data.

Each entry in the drum table can be in one of 8 queues which are used for optimization of transfers and retention of the most frequently used tracks on the drum. Four queues are assigned to the 4 disk modules, each queue containing all drum table entries which are requesting transfers on that modile. When a module finishes a transfer, its corresponding queue is searched for the next buffered transfer which will require the minimum disk head motion. If the program in core issues a CALL which forces a direct disk-to-core read, the optimization is abandoned to service the CALI.

Whenever an entry in the drum table is in the quiescent state, it is in one of 4 queues ( 2 for the single block area and 2 for the 8 block area entries). The higher priority queue contains entries with tracks which will probably be required shortly by some program. A second queue contains tracks which will probably not be needed again. If a drum block is needed 
to buffer a write, an entry from this second queue will be assigned the new track number. Only if this queue is empty will an entry from the higher priority queue be used. Whenever a drum block is used, the entry is placed at the bottom of the queue. whereas the entry removed was taken from the top. The effect of this procedure is to always keep the most frequently used track images near the bottom of the queue.

\subsection{Garbage Collection}

GCAFUL and REL $\phi A D$ were programmed to dump all of the $B \phi \phi \mathrm{TS}$ area from disk to tape and load it back up again. These were both one block programs and therefore not very efficient. This resulted in such a slow running program that tape transmission or other errors often occurred thereby requiring a re-bootstrap to finish the job which in turn caused a loss of pointers. Also it was noted that when using GCAFUL, one almost ran out of tape after dumping 175 cylinders. It was then decided that programs using three blocks were needed and thus DUMPIT and I $\varnothing$ ADIT were coded; they have not yet been debugged. These new dump programs write out a sumcheck and cylinder number after each cylinder is dumped and this information is then checked on reload. Also, two tapes will be used for holding the dumped information--one with 75 cylinders worth of data and the other with 100 .

GCl, which reloads the disk using consecutive cylinders, was programmed and is now being debugged. It has been observed that the speed of GC and GCl largely depended upon the format of the information which was put onto tape. Unfortunately, the format of GC does not lend itself to speed; each track is followed by its own sumcheck. Thus, upon reload, the computer is virtually inactive until both of these records are read in--making the program very $I / \phi$ dependent. Study of a different tape format would be desirable for any new garbage collectors.

\subsection{TRACE Routine Under B $\phi \phi \mathrm{TS}$}

While debugging a NICAP program with $B \phi \phi T S$, it is often desirable to get detailed traces of suspected portions of the program. If the NICAP routines mainly involve the manipulation of tables, one would like to have the facility of frequently checking the tables while the routine is running. A subroutine to accomplish the above was written for the purpose of debugging SYSDSK and should also prove useful in working with other untested programs of a similar nature. The program being checked sees the debugging 
rodtire as a simple trace.

Frogramming Memurandum No. 51, May 17, 1966, entitied "A FrovisionaI Irace and Tabie Debügging Routine for Time-Share Use" describes in detail the CALI sequence and parameters, the format and meaning of the output, trie furction of some of the takles in the TRACE routine, and the possible operation codes.

\subsection{PDP-7 Programming}

A temporary program was prepared for use in the debugging of the ILEIAC II time-sharing program. This program provides for the receipt of a. line of data from the IIIIAC II and the typing of this line on the PDP-? console.

The specifications for the ILLIAC-PDP-Consoie Interface have been determined and are set out in a memorandum distributed on April 18, 1966. They are basically as proposed and described in the last quarterly repcrt. The completion and means of interruption of both input and output lines has been decided.

There are to be three modes of operaticri.within the PUF-7. Tre first, is the Number mode which is the ncrmal mode of operation. This is the only mode in whi.ch commands to either the ILIIAC or the FDP-7 are recognized. Each line, uniess the line is a command, has a line number associated with it on koth input and output. On iriput, the first character in each line is a control character for the FDP 7 and will be interpreted as f'oliows:
a) First character $A \rightarrow Z$ indicates the line is a command to either the PDP-7, the ILLIAC, or both.
b) First character a space causes the PDP-7 to print the rext sequential line number followed by a space at which point tise users data line starts.
c) First character a period or $0 \rightarrow 9$ indicates the user is supplying his own line number.
d) First character a $\mathrm{CR}$ or $\mathrm{LF}$ is ignored.

The second mode of operation is the Data Mode. This is a special mode of operation in which no commands are recognized and all lines are sent to the ILLIAC as data. No line numbers are sent to the ILI.IAC in this mode, 
and similarly, no output line number is ever printed.

The third and last mode.within the PDP-7 is the tape mode. This is used to input paper tape from a console. It is similar to the Data Mode in that no commands are recognized but goes further by eliminating ALL response output from the PDP -7 .

There are certain commands which are recognized only by the PDP 7 , e.g. NUMBER A, B, DATA, TAPEN, TAPE D, TABS A, B, C, D, E. Other commands are shared by the PDP-7 and the ILLIAC, e.g. L $\phi \mathrm{GIN}$ and L $\phi \mathrm{G} \phi \mathrm{GT}$. All other commands are assumed to be for the ILIIAC and are sent to the ILIIAC as such.

The PDP-7-ILLIAC specifications were described last quarter and are fully laid out in Programming Memorandum No. 49. dated May 11, 1966.

The PDP-7 will receive, buffer, and transmit lines between the user and the ILLIAC II. Presently work is underway on the development of a program which will permit the use of 8 consoles connected to the PDP-7 in the time-sharing system. This program will in turn use the SPU PR $\phi$ GRAM INTERRUPT PR $\varnothing \mathrm{CESSOR}$ which simulates and processes all input-output which can be accomplished on the U. of I. PDP-7. Basically, the PR $\phi$ CESSOR program is a process by which specified core words are scanned. in order to determine which transfers to I/ $\varnothing$ devices have been completed. For example, initially input is requested from all consoles and the specified core words are then scanned to determine on which consoles input has been received. The consoles are serviced, input is again requested, and the scanning process continues. The I/ $\phi$ devices which are considered are the 630 communications network, the ILIIAC input and output channel, and the PDP-7 console teletypes.

\subsection{SHфRTTRAN}

A FØRTRAN-like compiler is being written for use from the remote consoles. The compiler is a single-pass type which results in an executable machịe language object program and. which remains in core during execution of the object program. This latter condition places restrictions on array size and limits object program length to about 2,500 words. FORTRAN statements are accepted by the compiler except for (at least initially) subroutine declarations. This language compiler is dubbed SH $\phi R T T R A N$ because of its FØRTRAN : origin and its limitations to short programs. An initial version of the compiler is coded and debugging has started. 
It is expected that users of SH $\phi$ RTTRAN will have higher priority than other users for the following reasons:

a) Single-pass compilation allows near immediate execution. (No intermediate $I / \phi$ occurs during compilation.)

b) Core swaps between SHфRTTRAN users are fast since not all of core needs moving.

Programming Memorandum No. 47, titled SHфRTTRAN, was distributed on April 18, 1966.

$\therefore \therefore$.

3.12 Graphical Display

The preliminary specification of the operating characteristics of the display console has been written. The characteristics of the internal construction of the software is currently in the planning stage and the first draft will soon be written. Since the computer system is not completely known, these specifications must be as machine independent as possible. In addition, this independence is desirable since the computer configuration may be changed once the project is under way. The design of the flow chart language is now taking place. The language specification and the internal construction of the software are closely related and thus must be planned together.

\subsection{Time-Sharing Hardware}

\subsubsection{Contest II.V.}

Contest II, the PDP-7 equivalent to ILIIAC II's AEC has become the standard engineering test program for the PDP-7. However, it had two problems. Contest II.V is merely Contest II with the problems fixed.

A pertinent publication this quarter was IOHGM-30 (Input-Output Hardware Group Memorandum) disbributed on May 17, 1966, and appropriately entitled "Contest II.V."

\subsubsection{Bootstrap Using Disk Only}

A bootstrap was programmed and wired using only the disk file. This was explained in detail in IOHGM-31, June 4, 1966, entitled "Bootstrap Using Disk Only.". This program is of importance to the time-sharing system. which is to be run from the disk file. 


\section{NUMERICAL METHODS, COMPUTER ARITHMETIC AND ARTIFICIAL LAIVGUAGES}

(Supported in part by the National Science Foundation under Grant No. NSF-GP-4636。)

\subsection{Autonomous Arithmetic Unit Siructures}

An approach suggested by $J$. E. Robertson has made it possible to synthesize minimal recoders for numbers in base two signed digit representation. The method, applicable to both left-directed and right-directed recodings; is to consider the recoding as being performed in two cascaded steps. The redundancy of the number representation is reduced as much as possible in the first step. This intermediate representation is then recoded by: a simpler, and hence easier to analyze, recoder.

If one assumes that all arithmetic values are equally likely (which is assumed in all work in this area of which the author is cognizant), one only need analyze the first step (assimilator) to the extent of identifying the arithmetically equivalent conventional digit sequence for each nonconventional digit sequence (one which contains a digit which is neither a zero or a plus one) in the intermediate representation. One can then claim that the shift average of the overall recoder is equal to that of the simpler recoder for numbers in conventional form if the output number representations of the simpler recoder have the same number of nonzero digits for each nonconventional digit sequence as for its conventional equivalent.

\subsubsection{Left-Directed Recoder}

It is well known that a left-directed assimilator can be implemented which can convert a signed-digit number to its conventional (nonredundant) form, and that this assimilator is not dependent on any digits to the left

of (i.e., with weighting factor greater than) the output digit. This assimilator will perform step one. The second step will be the canonical recoder, whose output is functionally dependent on one digit to the left of the output digit. By the argument presented above a minimal recoder has been thereby synthesized which is dependent on only one digit to the left of the output digit. 


\subsubsection{Right-Directed Recoder}

It is not possible to design a right-directed assimilator with finite functional dependence whose output is in nonredundant form. An assimilator was defined which converted base two signed-digit representations to nonredundent form except for the possibility of the inclusion of digit sequences consisting of a zero, followed by $K$ (where $K$ may be zero) ones, a two, and another zero. This sequence is arithmetically equivalent to a one followed by $(K+2)$ zeros. The output of this assimilator is functionally dependent on two digits to the right of the digit being processed.

The second step is performed by the minimal right-directed recoder determined by Penhollow ${ }^{1}$ extended to recode the intermediate representations described above. Analysis of all possible cases (six) of sequences including digits of value two indicate that the number of nonzero output digits is the same as for the equivalent conventional sequences. In four cases the output is independent of which input is applied; in the other two cases, more ones (and less minus ones) appear in the output for the nonconventional input representation than for the equivalent conventional representation. Therefore, although the shift average of the output representation is three for this recoder independent of the statistics of the input representation, its other statistics (except, of course, $P(0)$ ) are still functions of the statistics of the input representation. The output is functionally dependent on four digits to the right of the digit being processed.

The author combined both steps of this recoder into one step and examined the recoding table thus formed. In all but ten cases (of approximately 400) each case had an arithmetically symmetric pair; i.e., if all entries of a case are negated a case can be found with these entries. In each of these ten cases, a quasi-symmetric mate could be found (symmetric with respect to its input digits and mode digit only). In each of these cases, if the negative of the output of the quasi-symmetric mate were substituted for its own output, the number of nonzero output digits did not change for any digit sequence going through the altered case. These are therefore quasi-don'tcare conditions, which can be chosen to reduce the complexity of the decoder or to obtain desirable statistics in the output representation. (M. Pisterzi)

1. Penhollow, J. 0., "A Study of Arithmetic Recoding with Applications to Multiplication and Division," Department of Computer Science, University of Illinois, Urbana, Illinois 61801, Report No. 128, September 10, 1962. 


\subsection{Relationship Between Arithmetic Structure and Digital Probabilities}

While work has been continuing on the statistical analysis of the two adders described in the last progress report, another class of adders has been found. This class gives promise of greater flexibility than that offered by the adders mentioned previously in the sense that it may apparently be adapted, with variations, to handle a wide variety of radices and redundancies. Every adder of the class has the property that carry propogation is limited to a finite (and in practice, small) number of digital positions. One attractive and immediately obvious feature of the class is that al. internal signals are binary. Table I shows, as an example, a radix 2 signed-digit adder in. which both inputs and the output have digital values of $\bar{I}, \dot{0}, 1$. The sum digit, $s_{i}$, depends on digital positions $i, i+1$, $i+2$, and $i+3$ of the inputs as seen from the schematic. 


\begin{tabular}{|c|c|c|c|c|c|c|c|c|}
\hline$x_{i}+y_{i}$ & $t_{i-2}$ & $f_{i-1}$ & $\mathrm{w}_{\mathrm{i}}$ & $t_{i}$ & $f_{i}$ & $w_{i}$ & $t:$ & $\mathrm{w}_{i}^{\prime}$ \\
\hline$\overline{\overline{2}}$ & $\bar{I}$ & 1. & 0 & $\bar{I}$ & 0 & 0 & 0 & $\bar{I}$ \\
\hline $\bar{I}$ & $\bar{I}$ & $I$ & $I$ & $\bar{I}$ & 0 & 1 & 0 & 0 \\
\hline 0 & 0 & 0 & 0 & $\bar{I}$ & 1 & 0 & 0 & 0 \\
\hline 1 & 0 & 0 & 1 & $\bar{I}$ & 1 & 1 & 1 & $\bar{I}$ \\
\hline \multirow[t]{4}{*}{2} & 0. & .1 & 0 & 0 & 0. & 0 & 0 & 0 \\
\hline & & & & 0 & 0 & 1 & 1 & $\bar{I}$ \\
\hline & & & & 0 & 1 & 0 & 1 & $\bar{I}$ \\
\hline & & & & 0 & 1 & 1 & 1 & 0 \\
\hline
\end{tabular}

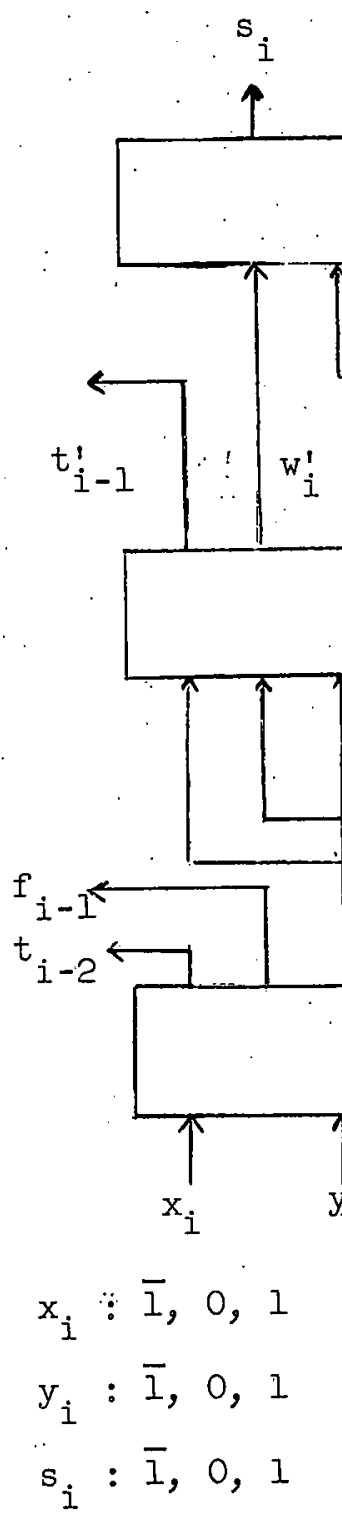

(F. Rohatsch) 


\section{ILLIAC II SERVICE, USE, AND PROGRAM DEVELOPMENT}

(This work is supported in part by Contract No. AT(11-1)-1469 of the Atomic Energy Commission and in part by the University of Illinois.)

\subsection{ILLIAC II Program Development}

\subsubsection{System Program Development}

Several modifications were made to the System Tape this quarter. Minor errors in the currently operating time-sharing system have been eliminated. Two extra blocks were added to the Batch Processor in order to incorporate an engineering test program into the daily running system. This ASMD (Add, Subtract, Multiply, Divide) test program is used as a background program in the time-sharing mode when code checks are not being run. Thus, even when no "batch" jobs are outstanding, the daily system may remain in operation and still provide a constant check of machine operation.

The Load-Procedure program was slightly modified in order to save pertinent registers before arriving at the conditional halt prior to the loading of a new program. Also added was a feature which (through a special register on the engineering-operator console) dynamically displays the user number and status of the program currently residing in core.

The Bootstrap and Load-Systems programs have been updated with changes which enable the immediate loading of a 5 block monitor prior to the loading of any other program. This insures the in-core residence of all necessary system programs at all times. The latest version of the special Register 34 Dumps will now work correctly. In order to condense the System Tape, the file which originally held the On-Line Monitor has now been deleted.

All the above system changes were made in order to provide better information for the engineers and a smoother running system.

The consulting service for the ILLIAC II has been a great help in relieving the system programmers from numerous questions. Since use of this service is rapidly increasing, thought is being given to expanding the available hours for consultation. 
The sections of Part are the responsibility of the following people:

\author{
Software System \\ Aaron; J: - Supervision and Maintenance \\ Brower, E. - Fortran Compiler \\ Slivinski, T. - Nicap and Macro \\ Richardson; F. K. - Batch Processor \\ Operations and Maintenance \\ Carter, C. - Supervision \\ Huffman, W., Lopeman, H. - Records \\ Kerkering, T. - CalComp Plotter \\ Conroy, D., Huszar, G. - Test Programming \\ C. W. Gear, Project Director
}

\title{
Library Development
}

\author{
Heiple, J: \\ Coane, $M$. \\ Chase, S.
}

L. D. Fósdick, Project Director

\subsubsection{FORTRAN, the ILLIAC II Compiler}

In preparation for the time-share version of F $\phi$ RTRAN, changes were made and debugged which allow for an eight block monitor system. The following things were implemented:

(a) a swtich in the location of the various buffers used by the i.ompiler,

(b) the inclusion of an input and output buffer for SYSFBN use,

and (c) a change of the CONST, VARIAB, DIMEN, and LABEL tables to a length of 128 words (rather than 256) with three 128 free half blocks linked for additional flexibility of the tables in the original amount of space.

A change was also made in Passes $I$ and II which enables the CEE (Common, Equivalence, and Error) table to stay in core (rather than being put onto the drum) as long as it does not exceed one block. The current system and 
the Math 195 system have been updated to incorporate these changes.

Presently coding for the time-share version of FøRTRAN involves switching from SYSI $\varnothing$ and SYSAUX to the new routines SYSFBN and SYSDSK.

The write up and flow charts for F $\phi$ RTRAN Pass 1, Part 1 were completed.

5.1.3. NICAP, the ILLIAC II Assembler, and the NICAP MACRO PREPASS

Severäl corrections and additions were made to NICAP. A particular address construction which was not assembling correctly is now fixed. Also, certain long NICAP jobs with many symbolic addresses were evolving and causing the "name" and "reference" tables to overflow and hang up the Assembler. Changes were made to Pásses I and IV in order to enable the correct assembling and the correct listing of tables for:such:jobs. : Minor changes to NICAP SYSERR were also required during this quarter.

An error in MACRO which caused any program containing macros and expanding to more than 900 cards to hang up during assembly has been corrected. other minor problems still exist and debugging continues.

Coding and testing of the changes required to make the NICAP assembler compatible with the new time-sharing system continue. 


\subsection{Library Programming}

\subsubsection{ILLIAC II Library Development}

During this quarter an error in the subroutine Bl-UOI-FATAN-45-NI was corrected. This necessitated a change in J5-UOI-CPIIEL-43-NI which was also made. Corrections were also made to the subroutines SQRT (FORTRAN), J5-UOI-CCP3NR-37-NI, and J5-UOI-CCP5AX-38-FR. The following subroutines were completed. (Some were not put onto the library tape until after the end of this quarter due to vacations and the fact that ILLIAC II was down for modifications to the building.)

E2-UOI-CFIT)

IDENTIFICATION--Chebyshev Curve-Fit for FORTRAN.

PURPOSE-Given an array of function values $Y(1), Y(2), \ldots, Y(M)$, and their arguments $X(I), X(2), \ldots, X(M)$, this subroutine will determine the array $A(1), A(2), \ldots, A(N+1)$ where the $A^{\prime}$ 's are the polynomial coefficients in the polynomial approximation

$$
P_{n}(x)=a_{1}+a_{2}+\ldots+a_{N+1} x^{N} \quad\left(a_{i} \equiv A(i)\right)
$$

to this tabulated function.

The criterion for this determination is the Chebyshev criterion; namely

$$
\max \left|P_{n}\left(x_{i}\right)-y_{i}\right| ; \quad\left(x_{1} \equiv X(I), y_{i} \equiv Y(I)\right), \quad i=1,2, \ldots, M,
$$

is a minimum for all possible choices of the $a_{i}$ 's. 
B 4-UOI-DPSQRI-64-NI

IDENTIFICATION--Double Precision Square Root for NICAP.

PURPOSE--To find the double precision square root of a given double precision number.

J5-UOI-LOGAX-67-FR

IDENTIFICATION--LOG AXIS for FORTRAN and NICAP.

PURPOSE--To draw an axis with a logarithmic scale for a graph to be plotted on the Calcomp plotter.

EI-UOI-HERMIT-70-NI

IDENIIFICATION--A. NICAP subroutine to perform Hermite interpolations.

PURPOSE--Given a table of $N$ abscissas, $x_{1}, \ldots, x_{N}$. with associated ordinates, $y_{1}, \ldots, y_{N}$ and derivatives

$$
\frac{d y_{1}}{d x_{1}}, \ldots, \frac{d y_{N}}{d x}
$$

and an arbitrary. point $x$, this subroutines uses Hermite interpolation to compute $y(x), \frac{d y(x)}{d x}$. 
IDENTIFICATION--A complete FORTRAN program to fit $N$ points to an equation of the form

$$
f(x)=a e^{b x^{2}}+c
$$

in the least squares sense.

PURPOSE-To compute a, b, $c$; so that a set of $N$ points $\mathrm{y}_{1}, \mathrm{y}_{2}, \ldots, \mathrm{y}_{\mathrm{N}}$ best fits in the least squares sense, the equation $f(x)=a e^{b x^{2}}+c$. At the option of the user the input points and graph of $f(x)$ are plotted on the same: plot, and or the $x_{1}, x_{2}, \ldots, x_{N} ; y_{1}, y_{2}, \ldots, y_{N} ; f\left(x_{2}\right), \ldots, f\left(x_{N}\right)$; $y_{1}-f\left(x_{1}\right), y_{2}-f\left(x_{2}\right), \ldots, y_{N}-f\left(x_{N}\right)$ are tabulated.

E2-UOI-EXPC-87-FR

IDENTIFICATION--A complete FORTRAN program to fit $N$ points to an equation of the form

$$
f(x)=a e^{b x}+c
$$

in the least squares sense.

PURPOSE--TO compute $a, b, c$, so that a set of $N$ points $\mathrm{y}_{1}, \mathrm{y}_{2}, \ldots, \mathrm{y}_{\mathrm{N}}$ best fits in the least squares sense, the equation $f(x)=a e^{b x}+c$. At the option of the user the input points and graph of $f(x)$ are plotted on the same plot, and or the 


$$
\begin{aligned}
& x_{1}, x_{2}, \ldots, x_{N} ; y_{1}, y_{2}, \ldots, y_{N} ; f\left(x_{1}\right), f\left(x_{2}\right), \ldots, f\left(x_{N}\right) ; \\
& y_{1}-f\left(x_{1}\right), y_{2}-f\left(x_{2}\right), \ldots, y_{N}-f\left(x_{N}\right) \text { are tabulated. }
\end{aligned}
$$

I. D. Fosdick, Project Director

S. Chase

M. Coane

J. Heiple 


\subsection{CalComp Digital Incremental Plotter}

The use of the CalComp increased by $1 / 3$ when the 7094 plotter output became available. Plotter output from the 7,094 started about mid April, 1966. April's Plotter use jumped to 270 hours from approximately 200 hours the month before. May's Plotter use was 321 hours. Even with the ILLIAC II shut down for the first three weeks of June, there were 186 hours of Plotter use in that month.

A full-time computer aid was: hired to take care of the daytime operation of the Plotter, and the four part-time student operators worked only in the evening and early morning hours. It now seems necessary to have another full-time operator for evening work and possibly some student help for early morning operation in order to keep output from backing up for more than 24 hours. The tapes for the last week of June were backed up for two and three days.

The Plotter break down time was again small. There were only six down times. in the three month period, four of which were due to broken chart drive springs: The longest down time occurred when the supply reel brake was out of adjustment. There have been no electronic failures in the system since we acquired it. Only minor mechanical failures have occurred.

The CalComp User's Group met twice in the last quarter. The second time was when the fast $780 / 763$ Plotting system was here for demonstration. Several small programs were run on the 7094, using the special Plot subroutine for comparison. It is estimated that in the worst case, the Zip Mode Plotter is a $t$ : least three times faster than the one we have now; in the ideal program, it is better than seven times faster. Besides being much faster, the 780/763 Plotting System's other features are simpler operation, much smoother straight and curved lines, and much easier servicing.

It is evident that more and more people are becoming interested in using the Plotter. If the present rate of increase in use is maintained, our present Plotter will be more than saturated in six months to a year.

From the results of the demonstration of the $780 / 763$ Plotting System, if another Plotting System is to be considered, fast turn around for a large volume of output could be maintained by the use of a fast system like the $780 / 763$ system for departmental users, and by using the 670/564 system we now have, for other department output. Operation of the two systems 
would not cost any more since supplies cost the same for both systems and both systems could be operated at the same time by one operator. The initial acquisition cost would be the major extra expense.

\subsection{Engineering Test Programming.}

In the second half of 1965 a system was planned which would take care of three types of engineering diagnostic testing:
(a) time-shared testing,
(b) single-program testing using magnetic tape, and
(c) diagnostic testing using paper tape:

The whole system is called the Automatic Engineering System (AES) and it consists of:
(1) ETS (Engineering Time-Sharing), similar to the AEC pre- sently operating,
(2) MTL (Magnetic Tape Library), similar to the old CREST system, and
(3) PTI (The Paper Tape Library).

The control program that acts as the monitor to ETS and as bookkeeper and editor to MTL and. PTL is AESM (the AES monitor).

In May and June of 1966 the ETS portion of AESM was completely checked out and debugged on ILLIAC. The MTL and PTL portions have been completely written but have not yet been checked out. 
5.5 Engineering Maintenance

5.5.1. ILLIAC II Engineering Log Summary - April, May, June, 1966

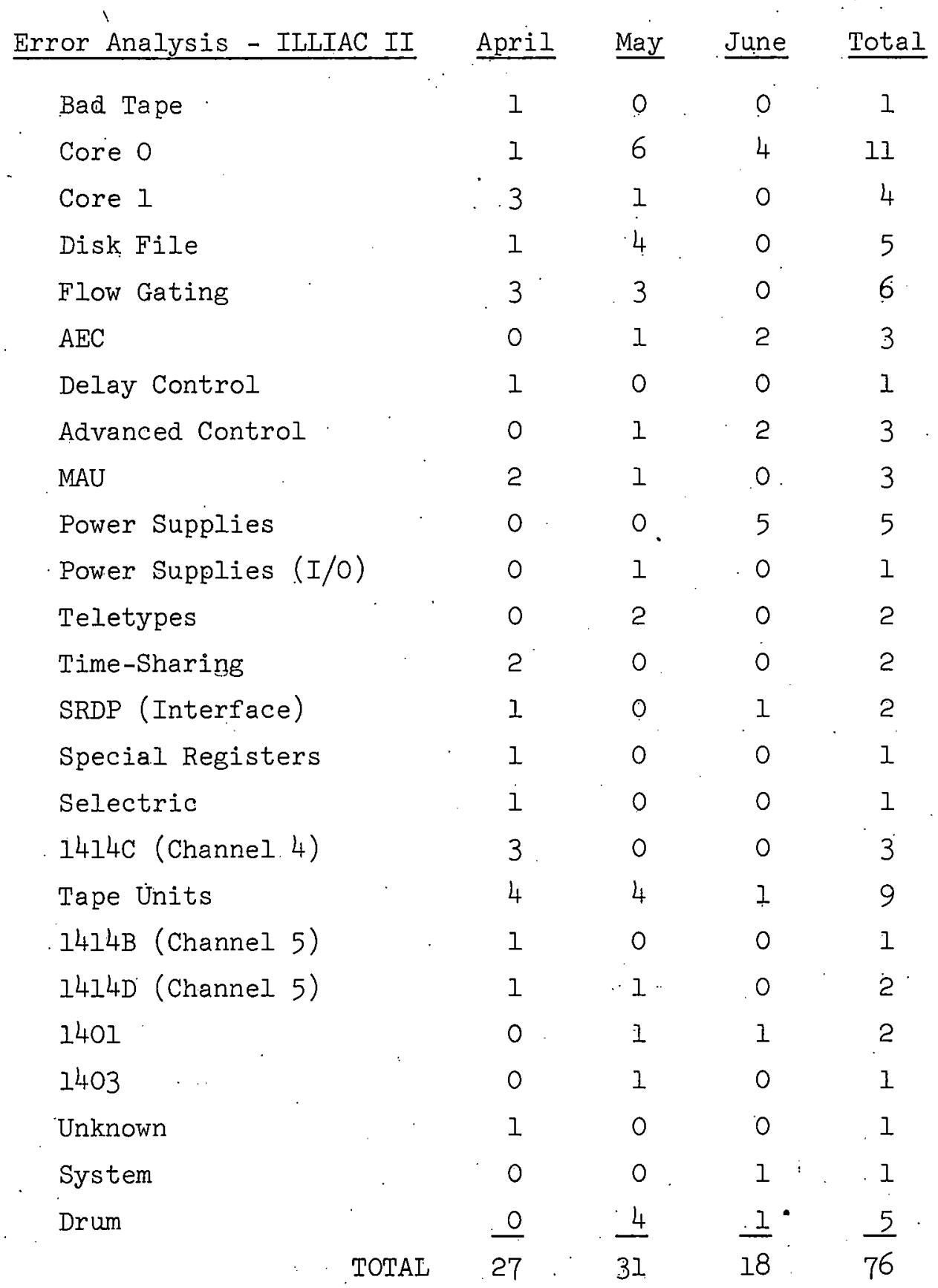

NOTE: Power was turned off all the machines at 0800 on May 27, 1966. The machines were wrapped to protect them from cement dust when the south walls were knocked out due to building expansion. Power was applied again on June 15, 1966. Due to the long down time that occurred the errors occurring between June 15-17, 1966, of which there were thirteen, will not be counted. 
5.5.2: Off-Line Equipment Requiring:Repairs

\begin{tabular}{|c|c|c|c|c|c|}
\hline Type & $\underline{\text { Name }}$ & April & May & June & Total \\
\hline 026 & Keypunch & 5 & 6 & 3 & 14 \\
\hline 026 & Intp. Keypunch & 0 & 1 & 2 & 3 \\
\hline 082 & Sorter & 1 & 1 & 0 & 2 \\
\hline 514 & Reproducer & 1 & 1 & 2 & 4 \\
\hline 557 & Alph. Interpreter & 0 & 1 & 0 & 1 \\
\hline 1056 & Reader & 2 & 3 & 2 & 7 \\
\hline 1057 & Keypunch & 0 & 1 & 0 & 1 \\
\hline \multirow[t]{3}{*}{1052} & Printer & 1 & 0 & 0 & 1 \\
\hline & TTY ASR & 4 & 2. & 4 & 10 \\
\hline & Data Sets & 0 & 2 & 0 & 2 \\
\hline
\end{tabular}

DCS Owned

$\begin{array}{lllllll}564 & \text { Plotter } & 0 & 1 & 1 & 2 \\ 670 & \text { Plotter Tape Unit } & 1 & 1 & 0 & 2 \\ & \text { TTY KSR } & 0 & \frac{1}{1} & \frac{2}{16} & \frac{3}{52}\end{array}$

\subsection{Scheduled Diagnostic Engineering}

5.6.1 Maintenance

a. Checking transistors

5 hours

b. Marginal DC voltage tests

11 . hours

c. Checking collector, base bumps and zeners

$2 \cdot$ hours

d. Component replacement tests

6 hours (trying new transistors, semiconductors, etc., to replace obsolete items)

e. Checking out spare chassis

3 hours

f. Checking and replacing card complements

2. hours

g. Checking spare cards that had been

20. hours modified for Core 0

h. SPU checkout

45 hours

i. $\mathrm{AC}$ and $\mathrm{DC}$ timing tests

3 hours 


\subsubsection{Component Failures}

a. Transistors in main machine (main frame, Core 1, interplay, drum, and power supplies)

$$
\begin{array}{cr}
\text { Main machine } & 40,121 \\
\text { Power supplies } & \frac{3,592}{43,713} \\
\text { TOTAL } & 46
\end{array}
$$

b. Semiconductors in main machine (diodes, zeners, stabistors; etc.)

TOTAL $\quad 81,946$

Semiconductors replaced 4

c. Number of printed circuit cards in I/O equipment

TOTAL

3,410

Cabinets not on line at present (1050, PAU, 1 1301B)

TOTAL

404

ON LINE TOTAL

3,006

Replaced

\subsubsection{Machine Modifications}

a. Removed terminations for 5-SET and 5-ACK on cable pallet coming from CF9-10 (top east side of Core 1 box). These signals were actually terminated twice.

b. Core O-noise on tester level/computer level used for on-line/ off-line operation. Added a $1 \mu f d$. capacitor to the inputs of all sense amplifier cards. This was completed on May 23, 1966.

c. 1301A-swapped leads in preparation of placing 1301B swing door on back of the 1301A cabinet. Cleaned up many temporary wires made the general appearance of the wiring side neater.

d. Removed the 1414A and 1414B cabinets from on-line operation and placed the Cable Distributor Cabinet in its position: $: \therefore$

e. Installed a 70 ampere circuit breaker to the $1301 \mathrm{~A}$ disk file. This was installed to allow initial run up to continue without tripping the circuit breaker.

f. ASMD troubles traced to more delay needed in advanced control at the base of 101 in Al6R. 
g. Inștalled 155-11 cards in D16-20 in 1414C; also in. D16, 17 in $1414 \mathrm{D}:$

h. Installed $156-29$ at $\mathrm{A} 14$ of $1414 \mathrm{C}$ and removed terminations at E14, 15, 17 of 1414D.

i. Removed local terminations in 1301A and 1414C for X-ACK and X-SET. The terminations are now located in Al5 of $1414 \mathrm{C}$.

$j$. A bleeder change was made on all the RS/WD cards in Core "O".

k. All regulators were wired so that a common place could be used to run the D.C. marginals of each box. The selector switches have not been installed.

5.6.4. Channel Interface Checkout

The SPU (Satellite Processing Unit) checkout was continued and at this time is $99 \%$ checked out.

\subsubsection{Transistor Replacements}

a. A search has been under way for an N-250 replacement. We have procured 150-N-250A's from Texas Instruments. Due to the inherent design of flow-gating these transistors are under test to determine whether they will actually replace the old $\mathrm{N}-250^{\prime}$.

b. We have received 200-S-166A's from Texas Instruments. These transistors are to replace the GF45011 and S-166's. Tests are now under way to determine whether they will actually be a good replacement.

\subsubsection{Causes for Computer Down Time}

a. The walls to the south of the main computer were knocked out during the month of May. A decision was reached that the cement dust would be too great to continue operation. On May 27 at 0800 the machine was powered down and wrapped up. The machine was not turned on until June 15, 1966.

b. The machine had to be shut off to install new water lines to the new building to the south at 2200 on April 2. The machine was turned back on at 0800 on April 4, 1966. 
c. On April 8, 1966, at 2051 the machine went down which was caused by an open word line in the Core 1 stack. This resulted in sixtythree hours of down time.

\subsection{Log Summaries}

5.7.1 ILLIAC II Use.

5.7 .1 .1 Summary of Use

\section{April, 1966}

Scheduled Engineering

$60: 40$

Unscheduled Engineering

$52: 32$

Engineering Development

119:42

Time-Sharing Development

$173: 40$

Power Off

Idle

Miscellaneous (operating, tape rewind, tape skipping, tape mounting, rerun of failing problems,

$9: 03$ starts of time-sharing)

TOTAL USE

Training and Education

$39: 18$

Training and Education (relinquish)

$: 22$

System Update

$2: 21$

System Dev.

$34: 47$

System Modification and Improvement

$34: 14$

Engineering Maint.

13:02

Customer Use

$\begin{array}{lr}\text { In System } & 48: 47 \\ \text { Special Short Shots } & : 30\end{array}$

Customer Use

49:17

Total Use

173:21

Total Time

220:00 


\section{May, 1966}

Scheduled Engineering

$50: 50$

Unscheduled Engineering

$53: 51$

Engineering Development

168:50

Time-Sharing Development

151:02

Power Off.

$110: 20$

Idle

Miscellaneous (operating, tape rewind, tape skipping, tape mounting, rerun of failures, starts of time-sharing)

TOTAL USE

Training and Education

$42: 10$

Training and Education (relinquish)

System Update

4:32

System Development

$53: 26$

System Modification and Improvement

$27: 51$

Engineering Maintenance

Customer Use

In Systems

$56: 27$

Special Short Shots

2:08
$58: 35$

Total Use

190:02

Total Time

$744: 00$ 
Scheduled Engineering

Unscheduled Engineering

Engineering :Development

Time-Sharing Development

Power Off $* 456: 00$

Idle

Miscellaneous (operating, tape rewind, tape skipping, $4: 49$ tape mounting, rerun of failures, starts of time-sharing)

TOTAL USE

Training and Education

Training and Education (relinquish)

System Update

System Development

System Modification and Improvement

Engineering Maintenance

Customer Use

In System

Customer Use

$10: 25$

$$
\begin{array}{r}
3: 41 \\
2: 15 \\
:: 37 \\
21: 52 \\
: 05 \\
8: 23
\end{array}
$$

10:25

Total Use

$47: 18$

Total Time

\footnotetext{
*Building construction forced a machine shut down for most of the month.
} 
5.7.1.2 Summary of Machine Errors

April, 1966

Main Machine

Disk Channel

Tape Channel

10

Selectric

$\stackrel{\frac{1}{26}}{=}$

$\mathrm{May}_{2} \cdot 1966$

Main Machine

15

Drum

4

Disk Channel

.4

Power Supplies

: 1

Tape Channel

$\frac{12}{36}$

June, 1966

Main Machine

18

Drum

2

Power Supplies

Tape Channel

$\underline{43}$ 


\section{Departmental Running Time}

April, 1966

Number of Runs

$\begin{array}{lrrr}\text { Dept. } & \text { T and Ed } & \text { Res } & \text { Total } \\ \text { AAE } & 832 & 133 & 965 \\ \text { ADV } & 17 & 3 & 20 \\ \text { AGE } & 14 & 3 & 17 \\ \text { AGEC } & 0 & 8 & 8 \\ \text { AGRON } & 0 & 75 & 75 \\ \text { ASTR } & 11 & 9 & 20 \\ \text { BOT } & 0 & 4 & 4 \\ \text { CCARCH } & 0 & 1 & 1 \\ \text { CE } & 107 & 5 & 112 \\ \text { CHE } & 37.0 & 48 & 418 \\ \text { DCS } & 782 & 363 & 1145 \\ \text { EE } & 617 & 184 & 801 \\ \text { IE } & 0 & 4 & 4 \\ \text { MATH } & 1784 & 33 & 1817 \\ \text { MATRL } & 0 & 182 & 182 \\ \text { ME } & 313 & 74 & 387 \\ \text { MMPE } & 0 & 2 & 2 \\ \text { NHS } & 0 & 36 & 36 \\ \text { IUCE } & 3 & 26 & 29 \\ \text { PHYCS } & 11 & 549 & 560 \\ \text { PSYCH } & 0 & 46 & 46 \\ \text { SWS } & 0 & 1 & 1 \\ \text { TAM } & 148 & 85 & 233 \\ \text { VPP } & 0 & 4 & 4 \\ \text { XSSS } & & 73 & 73 \\ \text { SUB TOTALS } & 5009 & 1951 & 6960 \\ \text { DCSSYS } & 228 & 919 & 1147 \\ \text { TOTALS } & 5237 & 2870 & 8107\end{array}$

Number of Specs

T. and Ed Res Total

\begin{tabular}{|c|c|c|}
\hline 11 & 6 & 17 \\
\hline 1 & 1 & 2 \\
\hline 14 & 1 & 15 \\
\hline 0 & 1 & $I$ \\
\hline 0 & 1 & 1 \\
\hline$I$ & 2 & 3 \\
\hline 0 & 1 & 1 \\
\hline 0 & 1 & 1 \\
\hline 1 & 2 & 3 \\
\hline 1 & 5 & 6 \\
\hline 4 & 7 & 11 \\
\hline 10 & 11 & 21 \\
\hline 0 & 1 & 1 \\
\hline 2 & 1 & 3 \\
\hline 0 & 4 & 4 \\
\hline 10 & 9 & 19 \\
\hline 0 & 1 & I \\
\hline 0 & 1 & 1 \\
\hline 1 & 2 & 3 \\
\hline 1 & 21 & 22 \\
\hline 0 & 2. & 2 \\
\hline 0 & 1 & 1 \\
\hline$: 5$ & 5 & 10 \\
\hline 0 & 1 & 1 \\
\hline 0 & 1 & 1 \\
\hline 62 & 89 & 151 \\
\hline$I$ & $11:$ & 12 \\
\hline 63 & 100 & 163 \\
\hline
\end{tabular}

\section{Usage in Hours}

9.473
.200

.200
.185

.000

.000

.087

.000

.000

.757

6.230

7.805

3.004

.000

5.367

.000

2.101

.000

.000

.012

.060

.000

.000

.883

000

.000

36.164

3.130

39.494
Res

1.966

:020

.053

.082

.571

.640

.164

.005

.091

.542

13.404

6.321

.046

5.396

1.986

.931

.012

.426

1.276

12.363

.186

.007

2.137

.152

.500

49.277

46.625

$95: 902$
Total

11.439

.220

.238

.082

.571

.727

.164

.005

.848

6.772

21.209

9.325

.046

10.763

1.986

3.032

.012

.426

1.288

12.423

.186

.007

3.020

.152

.500

85.441

49.755

135.196

$\mathrm{T}$ and $\mathrm{Ed}=$ Training and Education

Res $=$ Research 
May, 1966

Number of Runs

Number of Specs

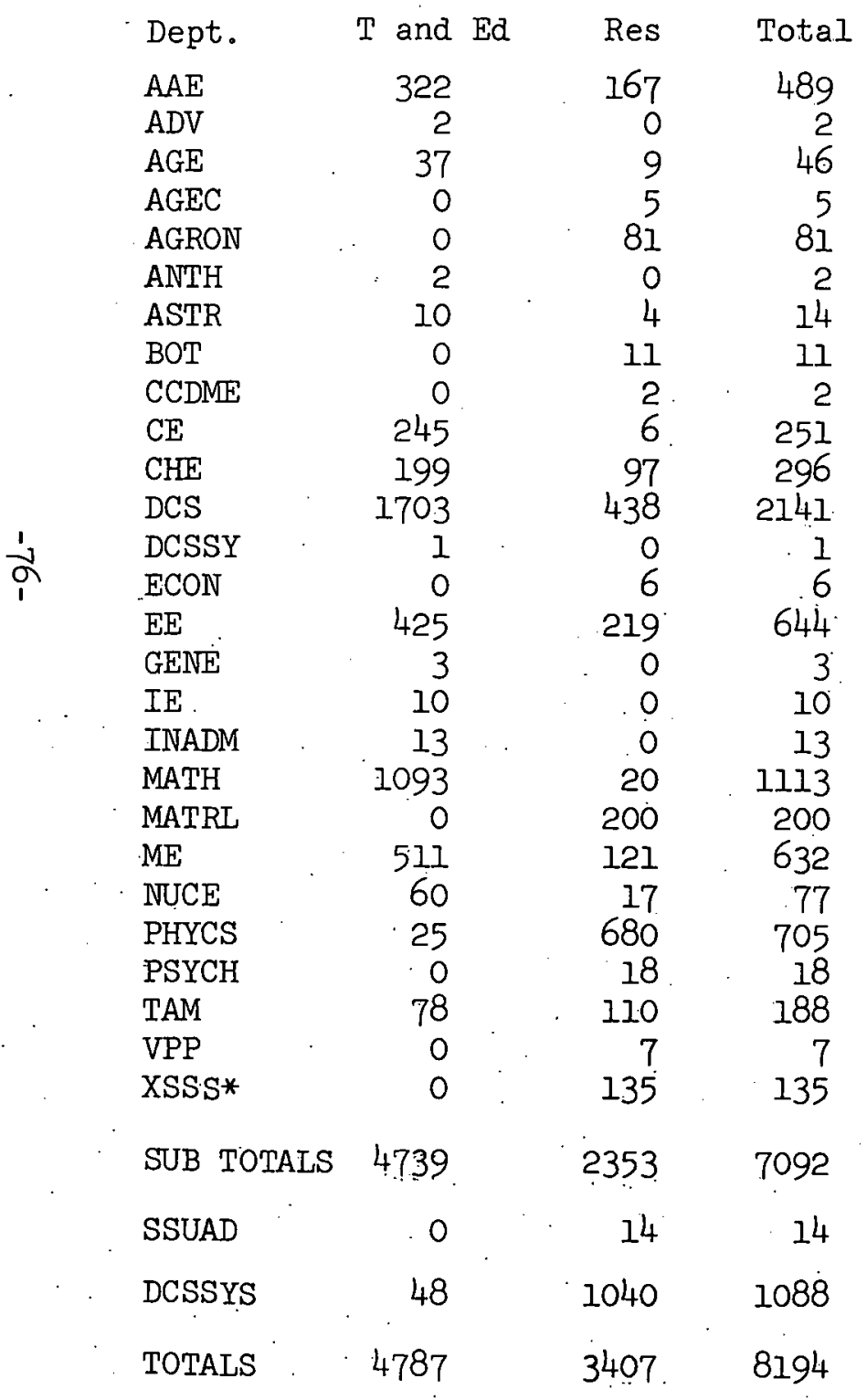

\begin{tabular}{|c|c|c|}
\hline $\mathrm{T}$ and $\mathrm{Ed}$ & Res & Total \\
\hline 8 & 5 & 13. \\
\hline 1 & 0 & 1 \\
\hline 1 & 1 & 2 \\
\hline 0 & 1 & 1 \\
\hline 0 & 1 & 1 \\
\hline 1 & 0 & 1 \\
\hline 2 & 1 & 3 \\
\hline 0 & 1 & 1 \\
\hline 0 & 1 & 1 \\
\hline 2 & 1 & 3 \\
\hline 1 & 6 & 7 \\
\hline 3 & 9 & 12 \\
\hline 1 & 0 & 1 \\
\hline 0 & $I$ & 1 \\
\hline 11 & 7 & 18 \\
\hline 1 & 0 & 1 \\
\hline 1 & 0 & 1 \\
\hline 1 & 0 & 1 \\
\hline 3 & 1 & .4 \\
\hline 0. & 4 & 4 \\
\hline 11 & 8 & 19 \\
\hline 3 & 3 & 6 \\
\hline 1 & 18 & 19 \\
\hline 0 & 1 & 1 \\
\hline 7. & 4 & 11 \\
\hline 0 & 1 & 1 \\
\hline 0 & 1 & 1 \\
\hline $5 \dot{9}$ & 76 & 135 \\
\hline 0 & 1 & 1 \\
\hline 1 & 8 & r \\
\hline 60 & 85 & 145 \\
\hline
\end{tabular}

Usage in Hours

$T$ and Ed Res Total

$\begin{array}{rrr}4.083 & 2.455 & 6.538 \\ .012 & .000 & .012 \\ .214 & .109 & .323 \\ .000 & .071 & .071 \\ .000 & .766 & .766 \\ .004 & .000 & .004 \\ .092 & .695 & .787 \\ .000 & .622 & .622 \\ .000 & .007 & .007 \\ 2.319 & .059 & 2.378 \\ 1.630 & 1.296 & 2.926 \\ 2.990 & 21.193 & 44.183 \\ .004 & .000 & .004 \\ .000 & .041 & .041 \\ 3.041 & 4.501 & 7.542 \\ .003 & .000 & .003 \\ .072 & .000 & .072 \\ .043 & .000 & .043 \\ 2.004 & 1.572 & 3.576 \\ .000 & 2.551 & 2.551 \\ 4.096 & 1.427 & 5.523 \\ .689 & .502 & .991 \\ .213 & .15 .381 & 15.594 \\ .000 & .102 & .102 \\ .661 & 3.165 & 3.826 \\ .000 & .091 & .091 \\ .000 & 2.141 & 2.141\end{array}$

42.170 $\quad 58.547 \quad 100.717$

$.000 \quad .099 \quad .099$

$\begin{array}{lll}.571 & 58.574 \quad 59.145\end{array}$

$42.741 \quad 117.220 \quad 159.961$ 
June, 1966

\begin{tabular}{lrrr} 
& \multicolumn{3}{c}{ Number of Runs } \\
Dept. & T and Ed & Res & Total \\
AAE & 23 & 24 & 47 \\
CE & 7 & 2 & 9 \\
CHE & 0 & 1 & 1 \\
DCS & 120 & 134 & 254 \\
ECON & 0 & 1 & 1 \\
EE & 5 & 33 & 38 \\
FOR & 0 & 6 & 6 \\
ILR & 0 & 2 & 2 \\
INADM & 2 & 0 & 2 \\
MATH & 103 & 0 & 103 \\
MATRL & 0 & 19 & 19 \\
ME & 10 & 7 & 17 \\
NUCE & 2 & 34 & 36 \\
PHYCS & 0 & 33 & 33 \\
PSYCH & 0 & 15 & 15 \\
TAM & 20 & 47 & 67 \\
XSSS & 0 & 5 & 5 \\
\hline 1 & & & \\
SUB TOTALS & 292 & 363 & 655 \\
& & & \\
DCSSYS & 0 & 553 & 553 \\
& & & \\
TOTALS & 292 & 916 & 1208
\end{tabular}

Number of Specs

$\begin{array}{ccc}\text { T and Ed } & \text { Res } & \text { Total } \\ 4 & 2 & 6 \\ 2 & 1 & 3 \\ 0 & 1 & 1 \\ 3 & 4 & 7 \\ 0 & 1 & 1 \\ 1 & 7 & 8 \\ 0 & 1 & 1 \\ 0 & 1 & 1 \\ 1 & 0 & 1 \\ 2 & 0 & 2 \\ 0 & 4 & 4 \\ 3 & 1 & 4 \\ 1 & 3 & 4 \\ 0 & 6 & 6 \\ 0 & 1 & 1 \\ 3 & 4 & 7 \\ 0 & 1 & 1 \\ 20 & 38 & 58 \\ 0 & 11 & 11 \\ 0 & 49 & 69\end{array}$

Usage in Hours

$\begin{array}{rrr}\text { T and Ed } & \text { Res } & \text { Total } \\ .721 & .561 & 1.282 \\ .089 & .010 & .099 \\ .000 & .013 & .013 \\ 2.103 & 6.725 & 8.828 \\ .000 & .006 & .006 \\ .029 & 1.072 & 1.101 \\ .000 & .025 & .025 \\ .000 & .025 & .025 \\ .008 & .000 & .008 \\ .474 & .000 & .474 \\ .000 & .201 & .201 \\ .145 & .052 & .197 \\ .011 & .808 & .819 \\ .000 & .287 & .287 \\ .000 & .094 & .094 \\ .109 & .511 & .620 \\ .000 & .024 & .024 \\ & & \\ 3.689 & 10.414 & 14.103 \\ .000 & .31 .272 & 31.272 \\ 3.689 & 41.686 & 45.375\end{array}$


5.7 .2 IBM 1401, 1402, 1403 Use

5.7.2.1 Summary of Use

April, 1966

Scheduled Engineering

$24: 45$

$17: 42$

$5: 46$

Maintenance

$15: 29$

Tape Test

$337: 49$

ILIIAC Preparation

$5: 47$

Other (listing, reproducing, reports, sys. copy, demonstrations, etc.)

$55: 46$

Power Off

$134: 40$

Idle

$122: 16$

Total

720:00

May, 1966

Scheduled Engineering

12:00

$25: 05$

Unscheduled Engineering

$6: 58$

Tape Test

$19: 33$

ILLIAC Preparation

Other (listing, autocoder; sy.s. copy, reproducing, Cobol, tape printing, etc.)

284:08

Power Off

$121: 24$

$113: 00$

Idle 161:52

Total

744:00

June, 1966

Scheduled Engineering

$2: 25$

Unscheduled Engineering

$8: 27$

$1: 23$

Maintenance

$9: 15$

Tape Test

$78: 38$

Other (listing, autocoder, sys:..copy; reproducing,

$58: 25$

Cobol, tape printing, etc.: $\because$

456:00

Power off

105:27

Idle

Total

720:00 
$=$

\subsubsection{Summary of Machine Errors}

April, 1966

1403 Printer

5

1401 Main Frame

2

Tape Unit

$\underline{1}$

Total $\underline{8}$

May, 1966

1401 Main Frame

1

1403 Printer

$\underline{4}$

Total 5

June; 1966

1401 Main Frame

2

1402 Reader-Punch

1

1403 Printer

2

729 Unit "L"

Total $\frac{1}{6}$ 


\section{IBM SERVICE, USE, AND PROGRAM DEVELOPMENT}

(Supported in part by the National Science Foundation under Grant No. NSF-GP-700).

\section{$6.1 \quad$ New Routines}

BI-UOI-ATAN-97-SR

Principal value of arctangent. For a given floating point number $X$, this program calculates arctangent $(X)$. This routine originated in the University of Michigan Executive System.

\section{Revised by Fran Benard \\ W. D. Marquis}

May 10, 1966

QO-UOI-CTN1-107-SR

Logical tape number substitution routine. The input/ output with conversion subroutines .TAPRD and .TAPWR, and all subroutines that execute F申RTRAN and MAD input/output statements referring to tapes, have the capability of substituting another logical tape number for the one supplied by the calling sequence. This subroutine sets up a table of pairs of tape numbers - a standard PORTHOS number to replace a given non-standard number. This table has the form expected by the system subroutine that makes the substitution. Programs which refer to logical tape numbers standard at another installation may most simply be made compatible with PORTHOS by use of this subroutine.

Programmed by H. George Friedman, Jr:

February 25, 1966 


\begin{abstract}
Bi-UOI-ARSIN-108-SR Arcsine and arccosine. For a given floating point number $X$, this program calculates arcsine $(X)$ or arccosine $(X)$. This program is adapted from the University of Chicago arcsine and arccosine routines.

Adapted by $T$. Wang

June 21; 1966
\end{abstract}

M2-UOI-DBI-IIO-SR

BCD integer to binary integer conversion routine. This program converts a one word BCD, fixed point decimal number to its binary equivalent. Leading blanks are ignored. This program was adapted from the University of Michigan Executive System.

Revised by Thomas Kuckertz June 10, 1966

QO-UOI-TIMI-II4-SR Timing routine. This routine allows the user to set up an internal clock and set, reset, and read it as desired. The clock internal to TIMI uses the system core clock stored in location 00005, which is stepped automatically once every 60th of a second. To reset the internal clock, the system clock is read and stored. At any point, the current reading of the internal clock is the difference between the current reading of the system clock and the stored start time。

Programmed by Martin Minow H. George Friedman, Jr. May 10, 1966

J5-UOI-CPBI-115-SR : Automatic CalComp buffer allocation. The CalComp plotter operates most efficiently when long records are written on the magnetic tape. Writing long records also significantly decreases 7094 execution time. This program allocates the unused area of memory for buffer space.

Programmed by John T. Bagwell, Jr. H. George Friedman, Jr. May 25, 1966 
B4-UOI-ISQRT-116-SR Floating point single precision complex square root. This routine computes the square root of the complex number $\mathrm{A}+\mathrm{Bi}$. This program is adapted from the University of Chicago routine ISQRT.

Revised. by W. D. Marquis

June 24, 1966 


$$
\begin{gathered}
\text { Table I - IBM } 1401-I \\
\text { Summary of Use } \\
\text { April, } 1966
\end{gathered}
$$

Scheduled. Engineering

Unscheduled Engineering

Maintenance

$10: 32$

7094 Preparation

$492: 24$

Listing

$22: 49$

Code Check

$5: 27$

Tape Dump

$5: 27$

Reproduction

$7: 25$

SMP

IILIAC II. Preparation:

Idle

Total

614:11.

Table II - IBM 1401-I

Simmary of Machine Errors

April, 1966

1401 Main Frame

1402 Card Reader Punch

11

1403 Printer

729 V Tape Drives

1
11
1
1
Total $\cdots$
14


Table I - IBM 1401-II

Summary of Use

April, 1966

Scheduled Engineer ing

$15: 40$

Unscheduled Engineering

$38: 50$

Maintenance

7094. Preparation

Listing

Code Check

Tape Dump

Reproduction

SMP

ILLIAC' II Preparation

Idle

\begin{tabular}{rr}
$15: 40$ \\
$38: 50$ \\
$10: 35$ \\
$426: 00$ \\
$19: 42$ \\
$6: 19$ \\
$7: 47$ \\
$3: 18$ \\
$30: 21$ \\
$6: 59$ \\
$14: 23$ \\
\hline Total \\
$\underline{585: 54}$
\end{tabular}

Table II - IBM 1401-II

Summary of Machine Errors

April, 1966

1402 Card Reader Punch

10

Total

$\underline{\underline{10}}$ 
Table I - IBM 7094

Summary of Use

April, 1966

Scheduled Engineering

$19: 54$

Unscheduled. Engineering

Maintenance

$11: 32$

Air Conditioning

Idle

Miscellaneous (Operator training, tape rewind, system tape mounting, rerun of failing

$80: 33$ problems, tape skipping, destruction of clock reading)

TOTAL USE

Training and Education

University Administrative Overhead Use

$32: 02$

$.14: 20$

System Modifications and Improvement

System Updating

Customer Use

In System

Relinquish

$281: 57$

AGEC

PHYCS

$24: 07$

$62: 12$

(Relinquish Total)

Special Short Shots

$86: 19$

Customer Use Total)

$1: 40$

369:56

Total Use $\quad 446: 49$

Total Time on 631:27

Table II - IBM 7094

Summary of Machine Errors

April, 1966

7607 Data Channel

729 Tape Drive

1301 Disk

Air Conditioner

$\begin{array}{ll} & 1 \\ & 1 \\ & 1 \\ & \frac{1}{4} \\ & \underline{4}\end{array}$ 
7094 Table III ApriI, 1966

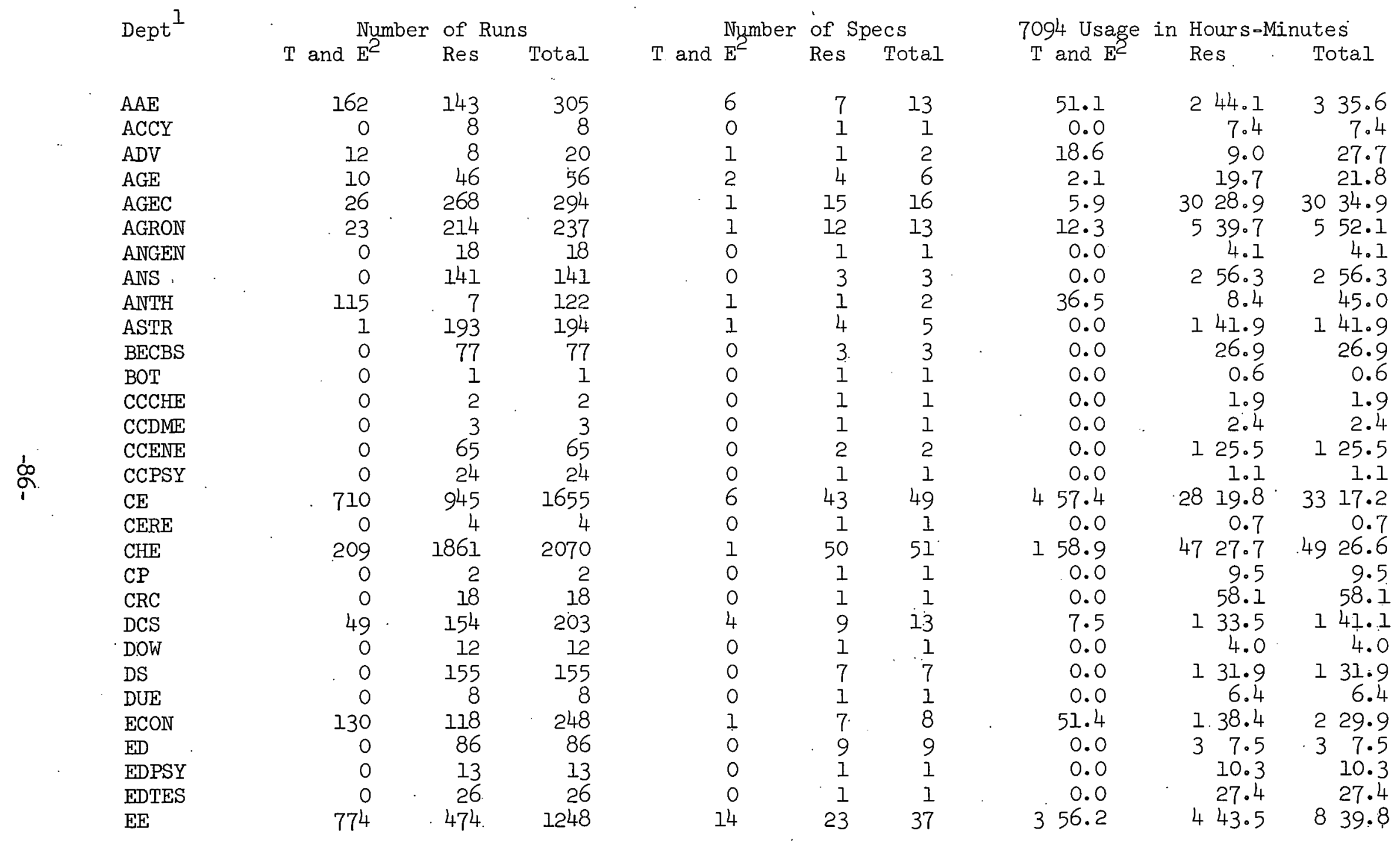


7094 Table III:April, 1966 Continued

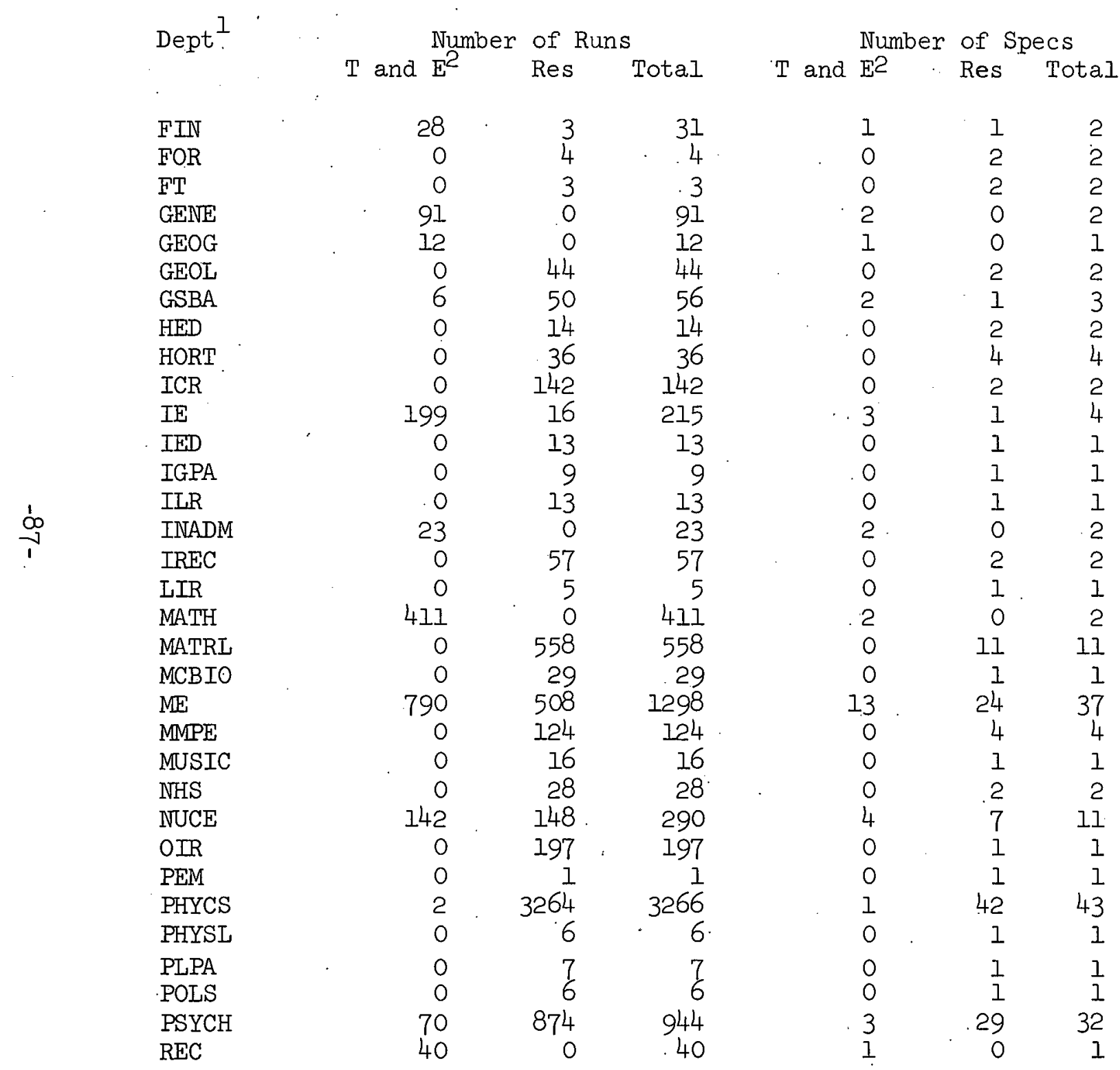

7094 Usage in Hours-Minutes $T$ and $E^{2}$ Res

Total

\begin{tabular}{|c|c|c|c|c|}
\hline 4.7 & & 0.4 & & 5.2 \\
\hline 0.0 & & 28.1 & & 28.1 \\
\hline 0.0 & & 1.0 & & 1.0 \\
\hline 53.5 & & 0.0 & & 53.5 \\
\hline 1.3 & & 0.0 & & 1.3 \\
\hline 0.0 & & 13.7 & & 13.7 \\
\hline 4.4 & & 44.0 & & 48.4 \\
\hline 0.0 & 1 & 14.9 & I & 14.9 \\
\hline 0.0 & & 30.7 & & 30.7 \\
\hline 0.0 & 3 & 22.2 & 3 & 22.2 \\
\hline 17.0 & & 31.6 & 1 & 38.6 \\
\hline 0.0 & & 11.5 & & 11.5 \\
\hline 0.0 & & 6.8 & & 6.8 \\
\hline 0.0 & & 22.9 & & $22 \cdot 9$ \\
\hline 15.7 & & 0.0 & & 15.7 \\
\hline 0.0 & & 31.1 & & 31.1 \\
\hline 0.0 & & 27.1 & & 27.1 \\
\hline 19.5 & & 0.0 & 1 & 9.5 \\
\hline 0.0 & 11 & 4.6 & 11 & 4.6 \\
\hline 0.0 & & 17.1 & & 17.1 \\
\hline 943.4 & 13. & 18.8 & 23 & $2 \cdot 3$ \\
\hline 0.0 & & 39.0 & & 39.0 \\
\hline 0.0 & & 21.4 & & 21.4 \\
\hline 0.0 & & 7.4 & & 7.4 \\
\hline 216.0 & 1 & 14.3 & 3 & 30.4 \\
\hline 0.0 & 5 & 53.3 & 5 & $53 \cdot 3$ \\
\hline 0.0 & & 0.4 & & \\
\hline 0.2 & 150 & 19.0 & 150 & 19.3 \\
\hline 0.0 & & 1.4 & & 1.4 \\
\hline $\begin{array}{l}0.0 \\
0.0\end{array}$ & & $\begin{array}{r}8.7 \\
28.1\end{array}$ & & $\begin{array}{r}8.7 \\
28.7\end{array}$ \\
\hline 26.6 & 15 & 2.7 & 15 & $29 \cdot 3$ \\
\hline 48.8 & & 0.0 & & \\
\hline
\end{tabular}


7094 Table III April, 1966 Continued

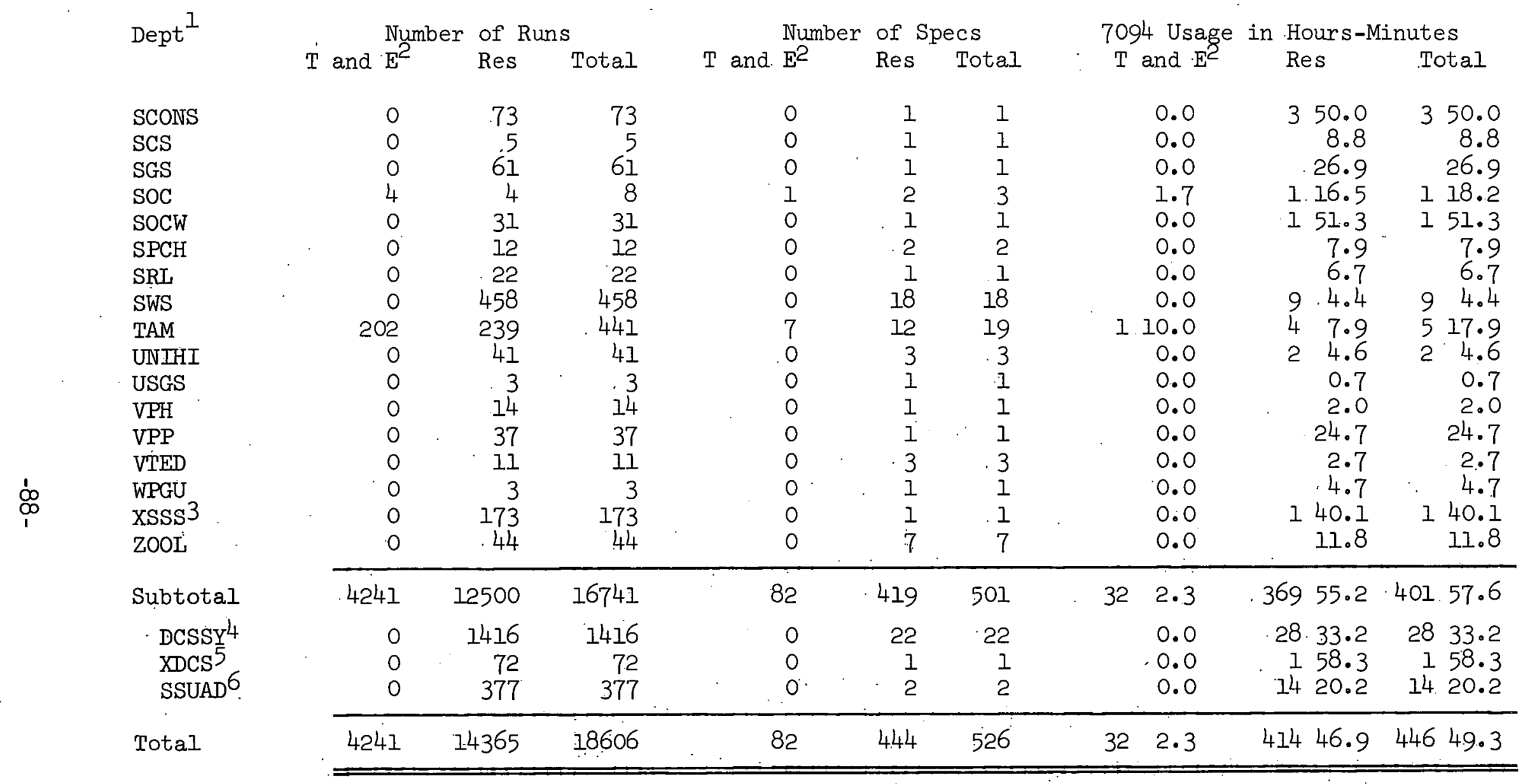

1 See list of departmental codes following

2 Training and Education

3 Special Short Shots

4 System Improvement and Modifications

5 System Updating

6 University Administrative Overhead Use 


$$
\begin{gathered}
\text { Table I - IBM } 1401-I \\
\text { Summary of Use } \\
\text { May, } 1966
\end{gathered}
$$

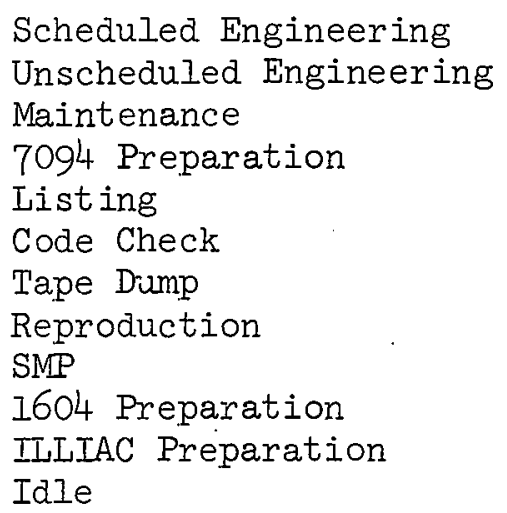

$$
\begin{aligned}
& \text { Table } \text { II - IBM } 1401-I \\
& \text { Summary of Machine Errors } \\
& \text { May, } 1966
\end{aligned}
$$

1403 Printer

729 V Tape Drives

Total $\frac{1}{1}$




\section{Table I - IBM .140I-II \\ Summary of Úse \\ May, 1966}

Scheduled Engineering

$7: 00$

Unscheduled Engineering

$14: 00$

Maintenaince

$14: 51$

7094 Preparation

Listing

Code Check

Tape Dump

Reproduction

SMP

IILIAC Preparation

Idle

$\begin{array}{r}7: 00 \\ 14: 00 \\ 14: 51 \\ 469: 56 \\ 19: 51 \\ 8: 42 \\ 6: 05 \\ 4: 10 \\ 43: 48 \\ : 50 \\ 26: 50 \\ \text { Total } \quad 616: 03 \\ \hline\end{array}$

Table II - IBM 1401-II

Summary of Machine Errors

May, 1966

1401 Main Frame

1402 Card Reader Punch

1

7

$\underline{\overline{8}}$ 
Table I - IBM 70.94

Summary of Use

May, 1966

Scheduled Engineering

$40: 06$

Unscheduled Engineering

$10: 19$

Maintenance

$17: 14$

Idle

Miscellaneous (Operator training, tape rewind, system

$3: 13$

tape mounting, rerun of failing problems,

tape skipping, destruction of clock

reading)

TOTAL USE

Training and Education

University Administrative Overhead Use

System Modification and Improvement

System Updating

$63: 36$

Customer Use

$\begin{array}{lr}\text { In System } & \\ \text { Relinquish } & \\ \text { AGEC } & 11: 51 \\ \text { EE } & : 24 \\ \text { OIR } & 1: 02 \\ \text { PHYCS } & 44: 51\end{array}$

Relinquish Total

Special Short Shots

Customer Use Total

$58: 08$

$: 38$

$70: 06$

$8:-41$

$24: 16$

$2: 00$

$298: 36$

$: 51$

$1: 02$

$44: 51$

$\underline{357: 22}$

Total Use

Total Time on
$462: 25$

$596: 53$

Table II - IBM 7094

Summary of Machine Errors

May, 1966

721 Card Punch

729 VI Tape Units

1301 Disk

7109 Central Processing Unit

7110 Instruction Processing Unit

7302 Core Storage

$\begin{array}{cc}1 \\ 1 \\ 2 \\ 1 \\ 1 \\ \text { Total } & 2 \\ 8\end{array}$


7094 Table III May 1966

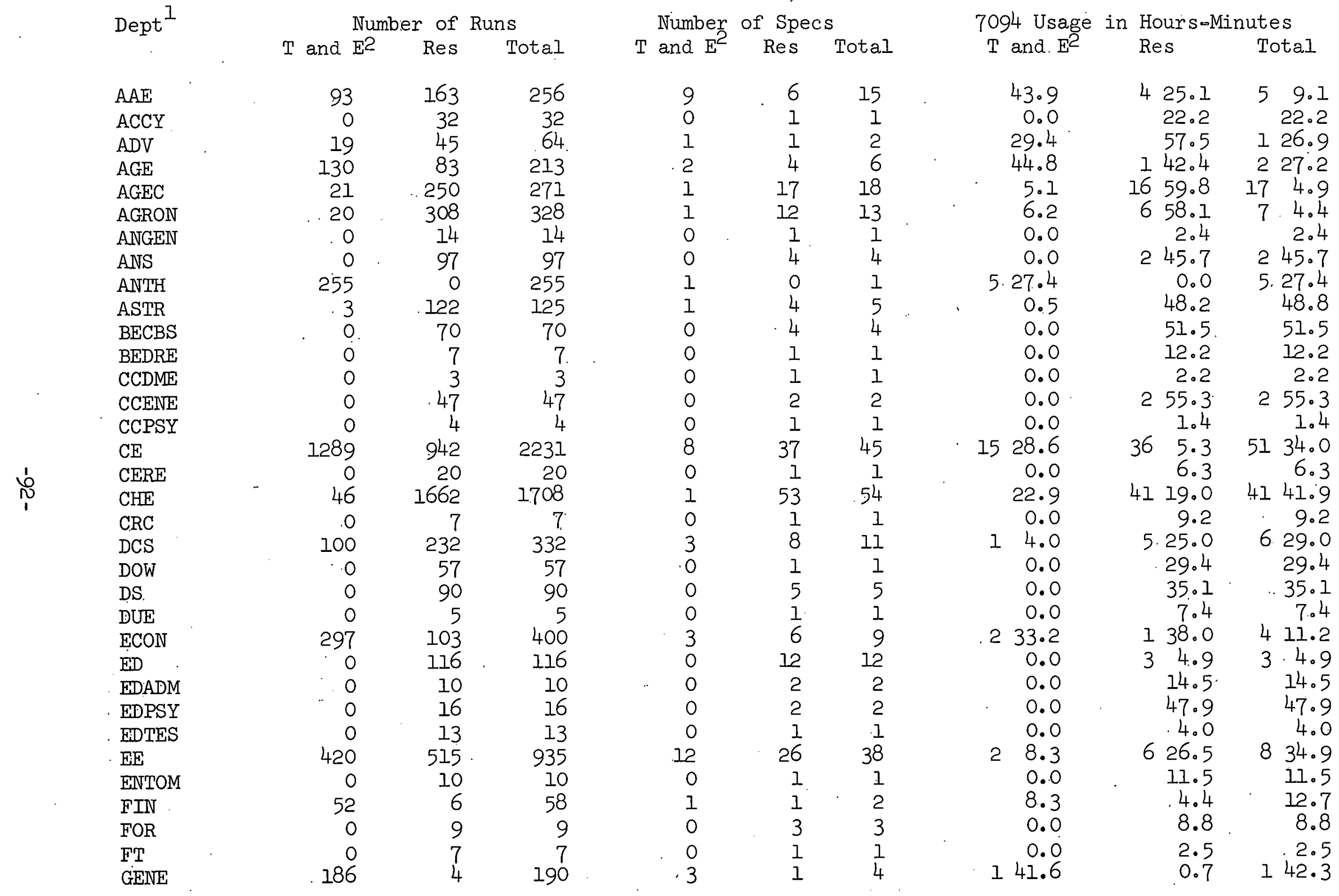


7094. Table III May 1966 Continued

\begin{tabular}{|c|c|c|c|c|}
\hline & Dept $^{1}$ & $\mathrm{Nu}$ & $r$ of & \\
\hline & & $\mathrm{T}$ and $\mathrm{E}^{2}$ & Res & Total \\
\hline & GEOG & 2 & 10 & 12 \\
\hline & GEOL & 0 & 51 & 51 \\
\hline & GSBA & 195 & 9 & 204 \\
\hline & HED & 0 & 3 & 3 \\
\hline & HORT & 0 & 36 & 36 \\
\hline & ICR & 0 & 128 & 128 \\
\hline - & IE & 287 & 5 & 292 \\
\hline & IGPA & 0 & 34 & 34 \\
\hline & INADM & 196 & 3 & 199 \\
\hline & IREC & 0 & 11 & 11 \\
\hline & LIR & 0 & 22 & 22 \\
\hline & MATH & 2357 . & 2 & 2359 \\
\hline & MATRL & 0 & 484 & 484 \\
\hline & MCBIO & 0 & 15 & 15 \\
\hline$\stackrel{\omega}{\omega}$ & $\mathrm{ME}$ & 815 & 676 & 1491 \\
\hline 1 & MMPE & 0 & 194 & 194 \\
\hline & MUSIC & 0 & 10 & 10 \\
\hline & NHS & 0 & 34 & 34 \\
\hline & NUCE & 295 & 177 & 472 \\
\hline & OIR & 0 & 239 & 239 \\
\hline & PEM & 0 & 47 & 47 \\
\hline & PHYCS & 0 & 3297 & 3297 \\
\hline & PHYSL & 0 & 1 & 1 \\
\hline & POLS & 0 & 17 & 17 \\
\hline & PSYCH & 152 & 1003 & 1155 \\
\hline & $\mathrm{REC}$ & 53 & 0 & 53 \\
\hline & SCONS & . 0 & 42 & 42 \\
\hline & SCS & 0 & 37 & 37 \\
\hline & SGS & 0 & 66 & 66 \\
\hline & SHCBR & 0 & 6 & 6 \\
\hline & $\mathrm{SOC}$ & 8 & 37 & 45 \\
\hline & SPCH & 0 & 14 & 14 \\
\hline & SRL & 0 & 53 & 53 \\
\hline & SWS & 0 & 544 & 544 \\
\hline & TAM & 400 & 175 & 575 \\
\hline
\end{tabular}

$\begin{array}{cl}\text { Number of Specs } \\ T \text { and } E^{2} & \text { Res Total }\end{array}$

$\begin{array}{rrr}1 & 1 & 2 \\ 0 & 2 & 2 \\ 2 & 1 & 3 \\ 0 & 2 & 2 \\ 0 & 4 & 4 \\ 0 & 2 & 2 \\ 4 & 1 & 5 \\ 0 & 2 & 2 \\ 2 & 1 & 3 \\ 0 & 3 & 3 \\ 0 & 1 & 1 \\ 2 & 1 & 3 \\ 0 & 11 & 11 \\ 0 & 1 & 1 \\ 14 & 24 & 38 \\ 0 & 8 & 8 \\ 0 & 1 & 1 \\ 0 & 2 & 2 \\ 6 & 6 & 12 \\ 0 & 1 & 1 \\ 0 & 3 & 3 \\ 0 & 43 & 43 \\ 0 & 1 & 1 \\ 0 & 3 & 3 \\ 4 & 29 & 33 \\ 1 & 0 & 1 \\ 0 & 1 & 1 \\ 0 & 1 & 1 \\ 0 & 1 & 1 \\ 0 & 1 & 1 \\ 2 & 4 & 6 \\ 0 & 5 & 5 \\ 0 & 2 & 2 \\ 0 & 16 & 16 \\ 8 & 10 & 18\end{array}$

7094 Usage in Hours-Minutes $T$ and $E^{2} \operatorname{Res}$ Total

\begin{tabular}{|c|c|c|c|}
\hline 0.2 & 3.7 & & 4.0 \\
\hline 0.0 & 27.8 & & 27.8 \\
\hline 612.7 & 26.4 & 6 & 39.2 \\
\hline 0.0 & 4.7 & & 4.7 \\
\hline 0.0 & 32.2 & & 32.2 \\
\hline 0.0 & 244.8 & 2 & 44.8 \\
\hline 225.8 & 8.9 & 2 & 34.8 \\
\hline 0.0 & $25 \cdot 3$ & & $25 \cdot 3$ \\
\hline 30.5 & $7 \cdot 3$ & & 37. \\
\hline 0.0 & 6.1 & & 6.1 \\
\hline 0.0 & 1.45 .2 & 1 & 45.2 \\
\hline 42.4 & 0.4 & 7 & 42.8 \\
\hline 0.0 & 629.2 & 6 & 29.2 \\
\hline 0.0 & 15.1 & & 15. \\
\hline 10.1 & 1749.7 & 22 & 0.0 \\
\hline 0.0 & 52.6 & & 52. \\
\hline 0.0 & 5.4 & & 5. \\
\hline 0.0 & 8.4 & & 0. \\
\hline 31.7 & 230.4 & 12 & 2.2 \\
\hline 0.0 & 853.6 & 8 & 53.6 \\
\hline 0.0 & 27.4 & & 27.4 \\
\hline 0.0 & 12740.7 & 127 & 40.7 \\
\hline 0.0 & 0.2 & & 0. \\
\hline 0.0 & 218.6 & 2 & 18. \\
\hline 150.9 & 2145.3 & 23 & 36. \\
\hline 8.3 & 0.0 & 1 & 8. \\
\hline 0.0 & $59 \cdot 3$ & & 59. \\
\hline 0.0 & $54 \cdot 3$ & & 54. \\
\hline 0.0 & 41.7 & & 41. \\
\hline 0.0 & 1.6 & & 1. \\
\hline 6.5 & 154.4 & 2 & O. \\
\hline 0.0 & 7.6 & & 7. \\
\hline 0.0 & 34.2 & & 34. \\
\hline 0.0 & 157.5 & 15 & 7. \\
\hline 21.4 & 127. & 0 & \\
\hline
\end{tabular}


7094 Table III May 1966 Continued

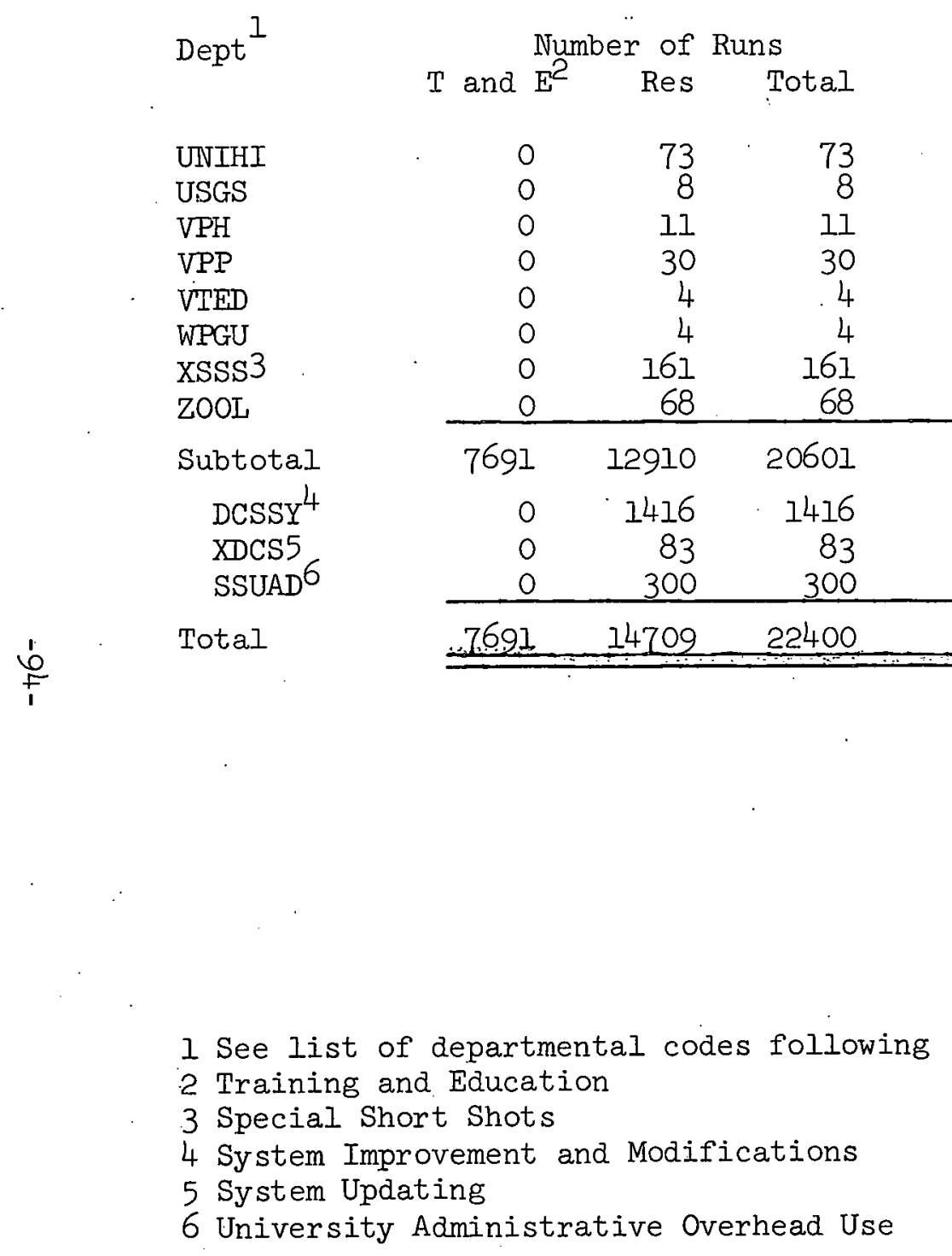

Number of Specs $T$ and $E^{2}$ Res Total $\begin{array}{lll}0 & 4\end{array}$

$\begin{array}{lll}0 & 4 & 4 \\ 0 & 1 & 1\end{array}$

0

0

$\begin{array}{lll}0 & 1 & 1 \\ 0 & 1 & 1\end{array}$

$\begin{array}{lll}0 & 1 & 1 \\ 0 & 2 & 2\end{array}$

$\begin{array}{lll}0 & 1 & 1\end{array}$

$\begin{array}{lll}0 & 1 & 1 \\ 0 & 1 & 1\end{array}$

0

$93 \quad 435$

7

$\begin{array}{lll}0 & 22 & 22\end{array}$

\begin{tabular}{lll}
0 & 1 & 1 \\
0 & 2 & 2 \\
\hline
\end{tabular}

$93 \quad 460 \quad 553$ 7094 Usage in Hours-Minutes
$\mathrm{T}$ and $\mathrm{E}^{2}$ Res

\begin{tabular}{|c|c|c|c|}
\hline & $\begin{array}{l}0.0 \\
0.0 \\
0.0 \\
0.0 \\
0.0 \\
0.0 \\
0.0 \\
0.0 \\
\end{array}$ & $\begin{array}{r}33.8 \\
4.4 \\
2.6 \\
22.4 \\
3.2 \\
6.8 \\
37.5 \\
24.1 \\
\end{array}$ & $\begin{array}{r}133.8 \\
4.4 \\
2.6 \\
22.4 \\
3.2 \\
6.8 \\
37.5 \\
24.1 \\
\end{array}$ \\
\hline 70 & 5.9 & 35721.0 & 42727.0 \\
\hline & $\begin{array}{l}0.0 \\
0.0 \\
0.0 \\
\end{array}$ & $\begin{array}{rr}24 & 16.2 \\
2 & 0.1 \\
8 & 41.4 \\
\end{array}$ & $\begin{array}{rr}24 & 16.2 \\
2 & 0.1 \\
8 & 4.14 \\
\end{array}$ \\
\hline
\end{tabular}

\footnotetext{
2 Training and Education

3 Special Short Shots

5 System Updating

6 University Administrative Overhead Use
} 


\section{TABLE I - IBM 1401-I \\ Summary of Use \\ June 1966}

Scheduled Engineering

Unscheduled Engineering

$: 30$

Maintenance

7094 Preparation

$13: 55$

List/Reproduce

Code Check

Tape Dump

SMP

Tape Testing

Idle

$3: 20$
$: 30$
$13: 55$
$499: 32$
$24: 34$
$13: 16$
$6: 54$
$18: 26$
$: 10$
$19: 46$
Total

Table II - IBM 1401-I

Summary. of Machine Errors

June 1966

729 V Tape Drives

$\underline{1}$

Total

$\stackrel{1}{=}$ 


\section{Table I - IBM 1401-II Summary of Use June 1966}

Scheduled Engineering $11: 30$ Unscheduled Engineering $5: 28$ Maintenance 7094 Preparation List/Reproduce Code Check Tape Dump

SMP

Tape Testing Idle

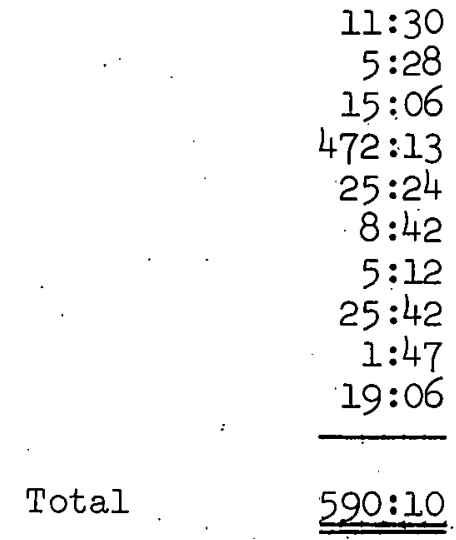

Table II.- IBM 1401-II Summary of Machine Errors June 1966

1402 Card Reader Punch

Total 


$$
\begin{gathered}
\text { Table I - IBM } 7094 \\
\text { Summary of Use } \\
\text { June } 1966
\end{gathered}
$$

Scheduled Engineering

Unscheduled Engineering

Maintenance

Air Conditioning

Idle

Miscellaneous (Or

(Operator training, tape rewind, system

TOTAL USE

Training and Education

University Administrative Overhead Use

System Modification and Improvement

System Updating

Customer Use

$$
\text { In system }
$$

Relinquish

AGEC

$\mathrm{EE}$

OIR

PHYCS

Special Short Shots

Customer Use Total
$332: 37$

$32: 59$

$1: 21$

$2: 08$

38:36

407:55

Total Use

461:15

Total Time On

$653: 47$

\footnotetext{
Table II - IBM 7094

Summary of Errors

June 1966
}

729 VI Tape Unit

7109 Arithmetic Sequence Unit.

Clear Button

716 Printer

Air Conditioner

$\begin{array}{ll} & 1 \\ & 1 \\ & 1 \\ 1 & 1 \\ 1 & \\ & \stackrel{5}{1}\end{array}$


70.94 Table III June 1966

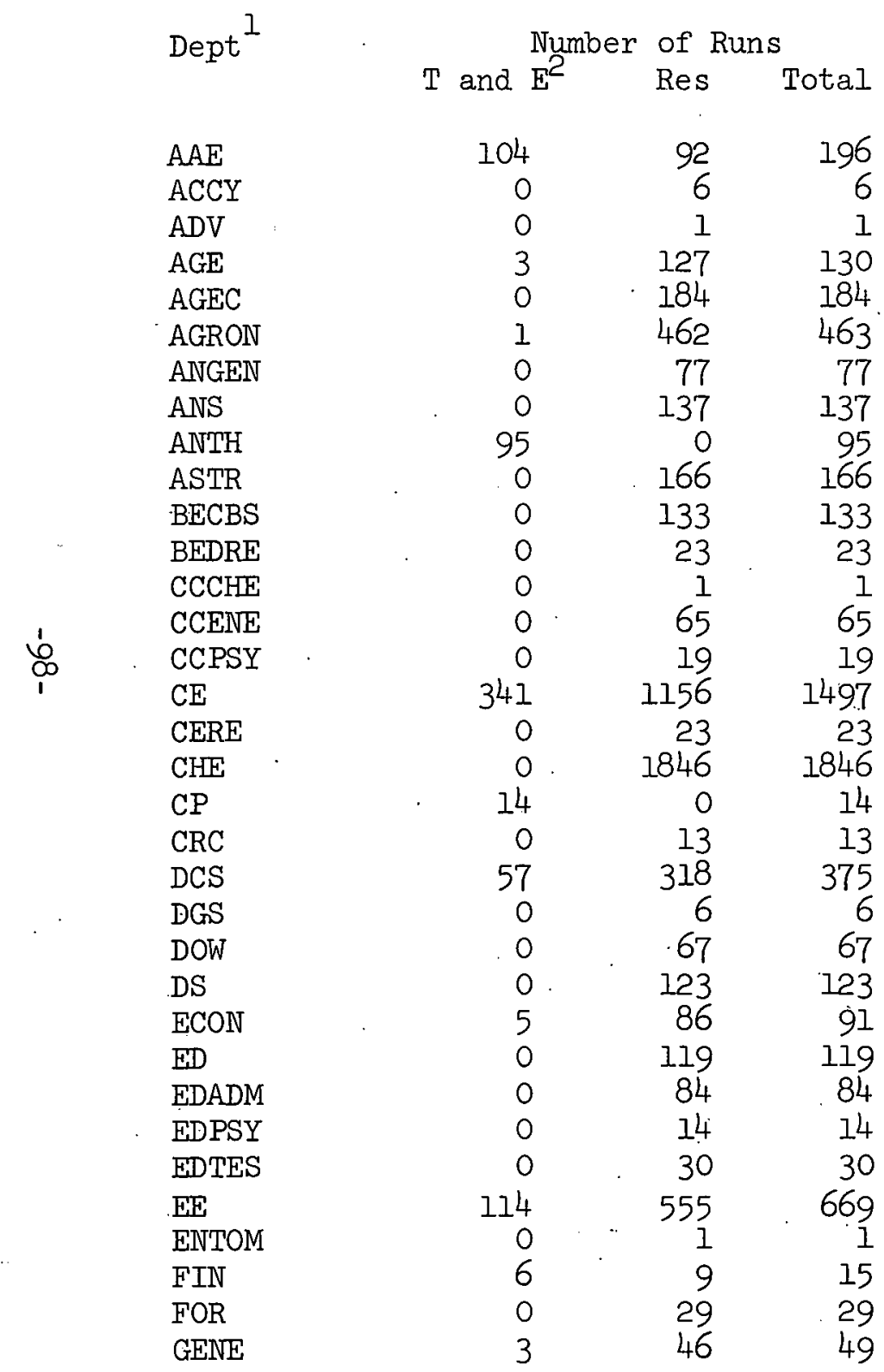

Number of Specs $\mathrm{T}$ and $\mathrm{E}^{2}$ Res Total

$\begin{array}{rrr}5 & 6 & 11 \\ 0 & 1 & 1 \\ 0 & 1 & 1 \\ 1 & 5 & 6 \\ 0 & 16 & 16 \\ 1 & 14 & 15 \\ 0 & 1 & 1 \\ 0 & 4 & 4 \\ 1 & 0 & 1 \\ 0 & 4 & 4 \\ 0 & 3 & 3 \\ 0 & 1 & 1 \\ 0 & 1 & 1 \\ 0 & 1 & 1 \\ 0 & 1 & 1 \\ 5 & 40 & 45 \\ 0 & 1 & 1 \\ 0 & 45 & 45 \\ 1 & 0 & 1 \\ 0 & 1 & 1 \\ 2 & 10 & 12 \\ 0 & 1 & 1 \\ 0 & 1 & 1 \\ 0 & 5 & 5 \\ 2 & 5 & 7 \\ 0 & 10 & 10 \\ 0 & 2 & 2 \\ 0 & 2 & 2 \\ 0 & 1 & 1 \\ 5 & 20 & 25 \\ 0 & 1 & 1 \\ 1 & 1 & 2 \\ 0 & 3 & 3 \\ 1 & 2 & 3\end{array}$

7094 Usage in Hours-Minutes $\mathrm{T}$ and $\mathrm{E}^{2}$ Res Total

\begin{tabular}{|c|c|c|c|}
\hline \multicolumn{2}{|c|}{118.1} & 220.5 & 338.6 \\
\hline & 0.0 & 1.7 & 1.7 \\
\hline & 0.0 & 0.0 & 0.0 \\
\hline & 0.5 & 114.5 & 115.0 \\
\hline & 0.0 & $36 \quad 5.1$ & 36 \\
\hline & 0.2 & 855.8 & 856.1 \\
\hline & 0.0 & 16.7 & 16.7 \\
\hline & 0.0 & 116.7 & 116.7 \\
\hline & 24.8 & 0.0 & 24.8 \\
\hline & 0.0 & 113.1 & 113.1 \\
\hline & 0.0 & 119.0 & 119.0 \\
\hline & 0.0 & 43.9 & 43. \\
\hline & 0.0 & 0.1 & 0.1 \\
\hline & 0.0 & 223.1 & 223.1 \\
\hline & 0.0 & $12 \cdot 3$ & $12 \cdot 3$ \\
\hline & 15.2 & $48 \quad 9.5$ & 5724 \\
\hline & 0.0 & $5 \cdot 3$ & $5 \cdot 3$ \\
\hline & 0.0 & 4131.7 & 4131.7 \\
\hline & 11.6 & 0.0 & 11.6 \\
\hline & 0.0 & 10.9 & 10.9 \\
\hline 2 & 6.3 & 731.6 & 938.0 \\
\hline & 0.0 & 5.0 & 5.0 \\
\hline & 0.0 & 39.1 & 39.1 \\
\hline & 0.0 & 39.5 & 39. \\
\hline & 0.8 & 250.2 & 251. \\
\hline & 0.0 & 243.1 & 243.1 \\
\hline & 0.0 & 23.4 & 23.1 \\
\hline & 0.0 & 38.9 & 38. \\
\hline & 0.0 & 13.5 & $13 \cdot 5$ \\
\hline 2 & $\begin{array}{l}3.2 \\
0.0\end{array}$ & $8 \quad 16.1$ & $10 \quad 19.3$ \\
\hline & 0.7 & 6.6 & 7.4 \\
\hline & 0.0 & 155.5 & 155.5 \\
\hline & 0.7 & 25.0 & 25.7 \\
\hline
\end{tabular}


7094 Table III June 1966 Continued

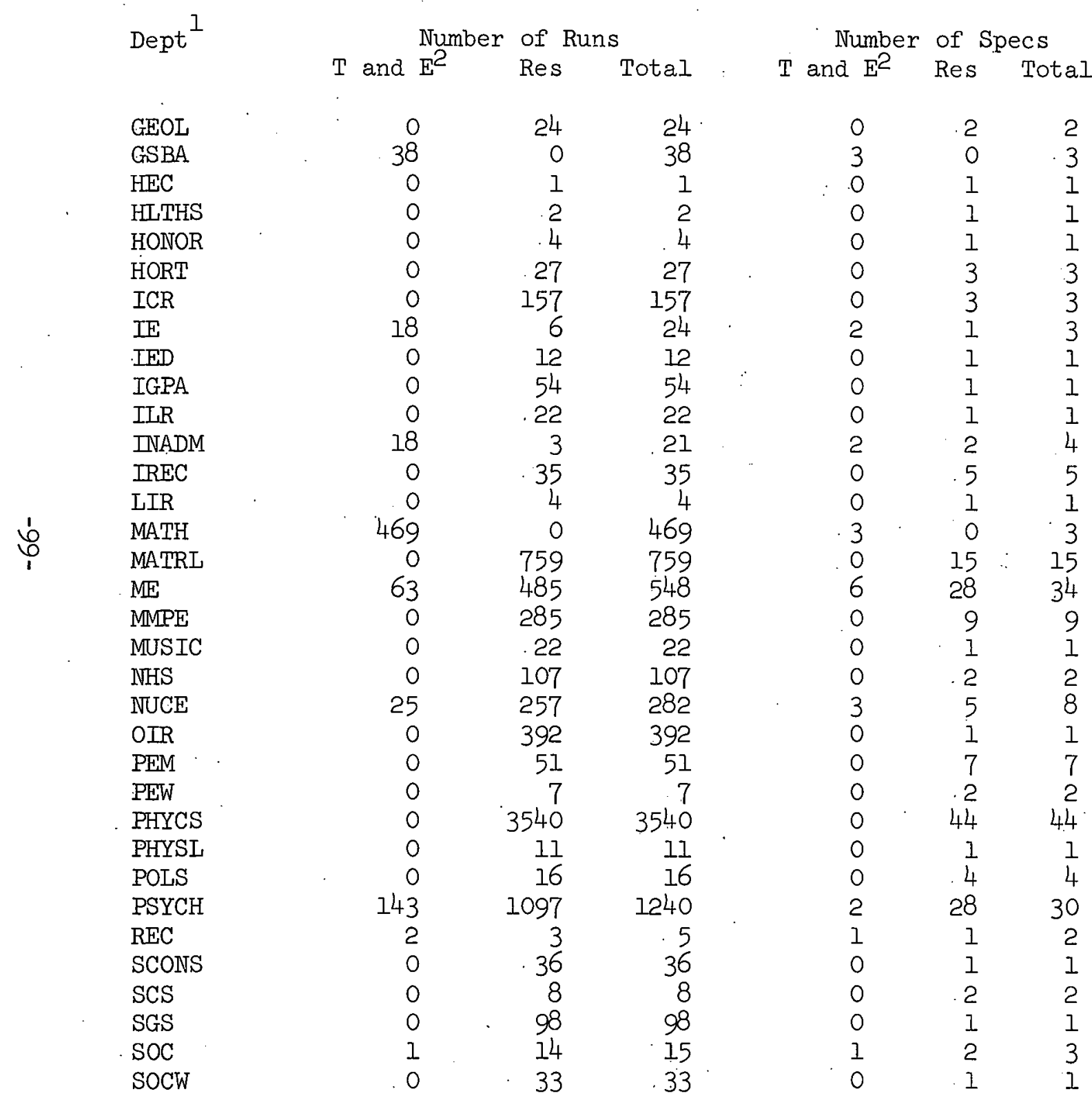

70.94 Usage in Hours-Minutes $\mathrm{T}$ and $\mathrm{E}^{2}$ Res Total

\begin{tabular}{|c|c|c|c|}
\hline 0.0 & 19.6 & & 19.6 \\
\hline 28.6 & 0.0 & & $\begin{array}{r}28.6 \\
1.7\end{array}$ \\
\hline 0.0 & 1.7 & & 1.9 \\
\hline $\begin{array}{l}0.0 \\
0.0\end{array}$ & $\begin{array}{l}15.4 \\
42.4\end{array}$ & & 42.4 \\
\hline 0.0 & 9.9 & & 9.9 \\
\hline 0.0 & 36.2 & 3 & 6.2 \\
\hline 13.7 & 1.7 & & 15.5 \\
\hline 0.0 & 11.9 & & 11.9 \\
\hline 0.0 & 11.5 & & 11.5 \\
\hline 0.0 & 47.7 & & 47.7 \\
\hline 13.0 & 9.2 & & 22.2 \\
\hline 0.0 & 110.2 & 1 & 10.2 \\
\hline 0.0 & 31.7 & & 31.7 \\
\hline 41.1 & 0.0 & 1 & $4 I .1$ \\
\hline 0.0 & 112.0 & 11 & 2.0 \\
\hline 28.1 & 1025.1 & 10 & $53 \cdot 3$ \\
\hline 0.0 & 524.1 & 5 & 24.1 \\
\hline 0.0 & 6.2 & & 6.2 \\
\hline 0.0 & 52.6 & & 52.6 \\
\hline 5.2 & 3.49 .8 & 3 & 55.1 \\
\hline 0.0 & 1331.7 & 13 & 31.7 \\
\hline 0.0 & 47.8 & & 47.8 \\
\hline 0.0 & $8 \cdot 3$ & & $8: 3$ \\
\hline 0.0 & $13623 \cdot 3$ & 136 & $23 \cdot 3$ \\
\hline 0.0 & 3.8 & & 3. \\
\hline 0.0 & 22.9 & 2 & 2.9 \\
\hline 6.5 & 2241.8 & 23 & 48.4 \\
\hline 30.7 & 1.1 & & 31.9 \\
\hline 0.0 & 6.1. & & 6.1 \\
\hline 0.0 & 6.1 & & 6.1 \\
\hline 0.0 & 25.3 & & 25.3 \\
\hline 0.1 & 10.4 & & 10.6 \\
\hline 0.0 & 115.8 & 1 & 15.8 \\
\hline
\end{tabular}


7094 Table III June 1966 Continued

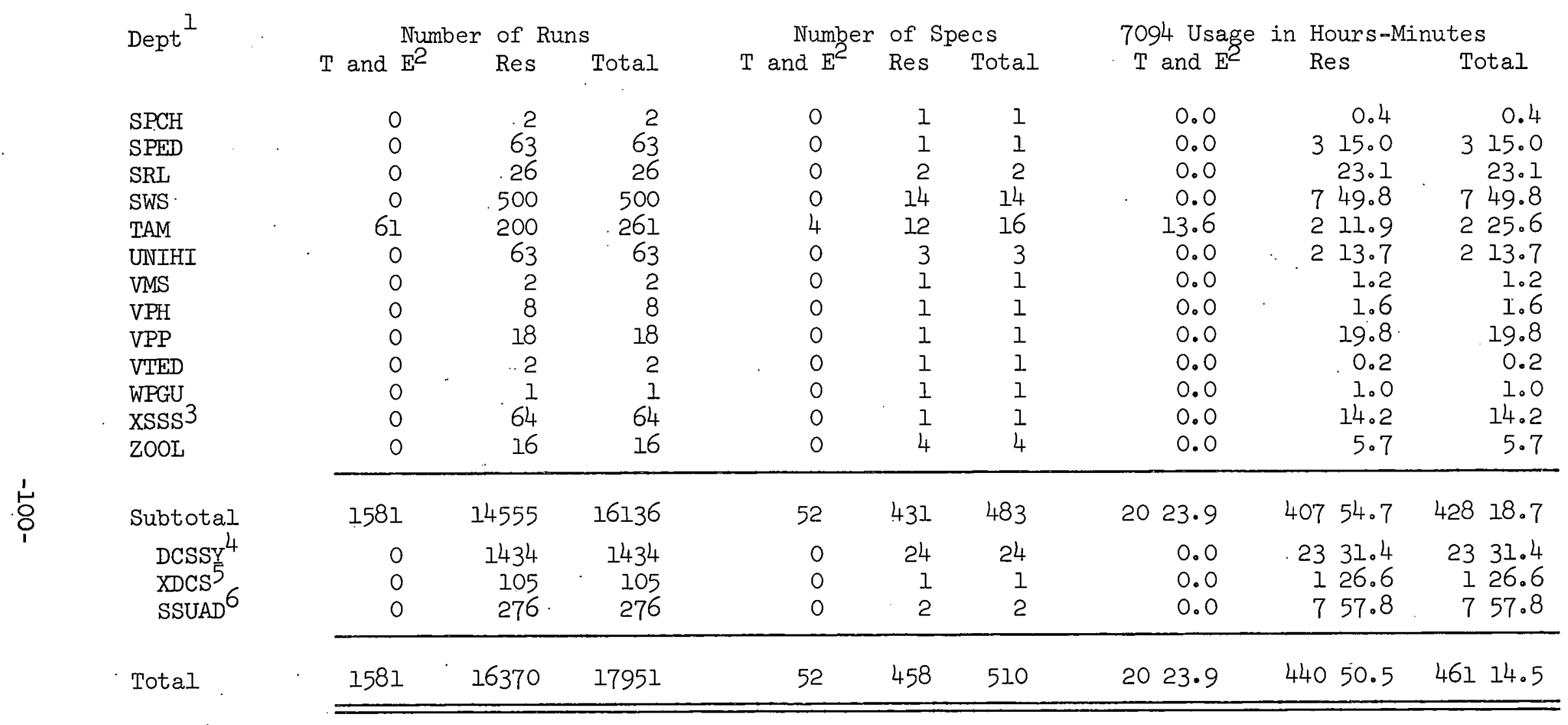

1 See list. of departmental codes following

2 Training and Education

3 Special Short Shots

4 System Improvement and Modifications

5 System Updating

6 University Administrative Overhead Use 
Quarterly Summary of Departmental Running Time

April, May, June, 1966

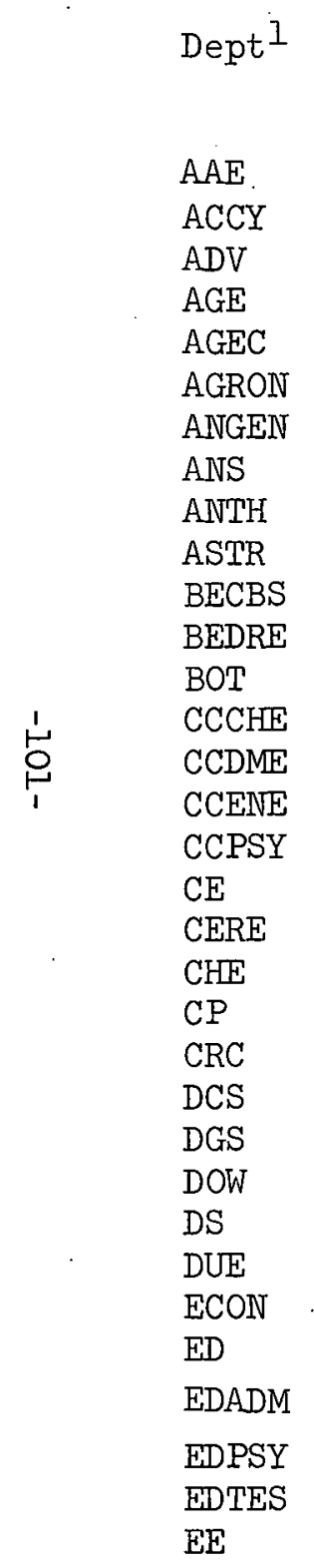

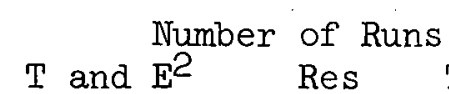

Total

Number of Specs

$\mathrm{T}$ and $\mathrm{E}^{2}$ Res Total

7094 Usage in Hours-Minutes

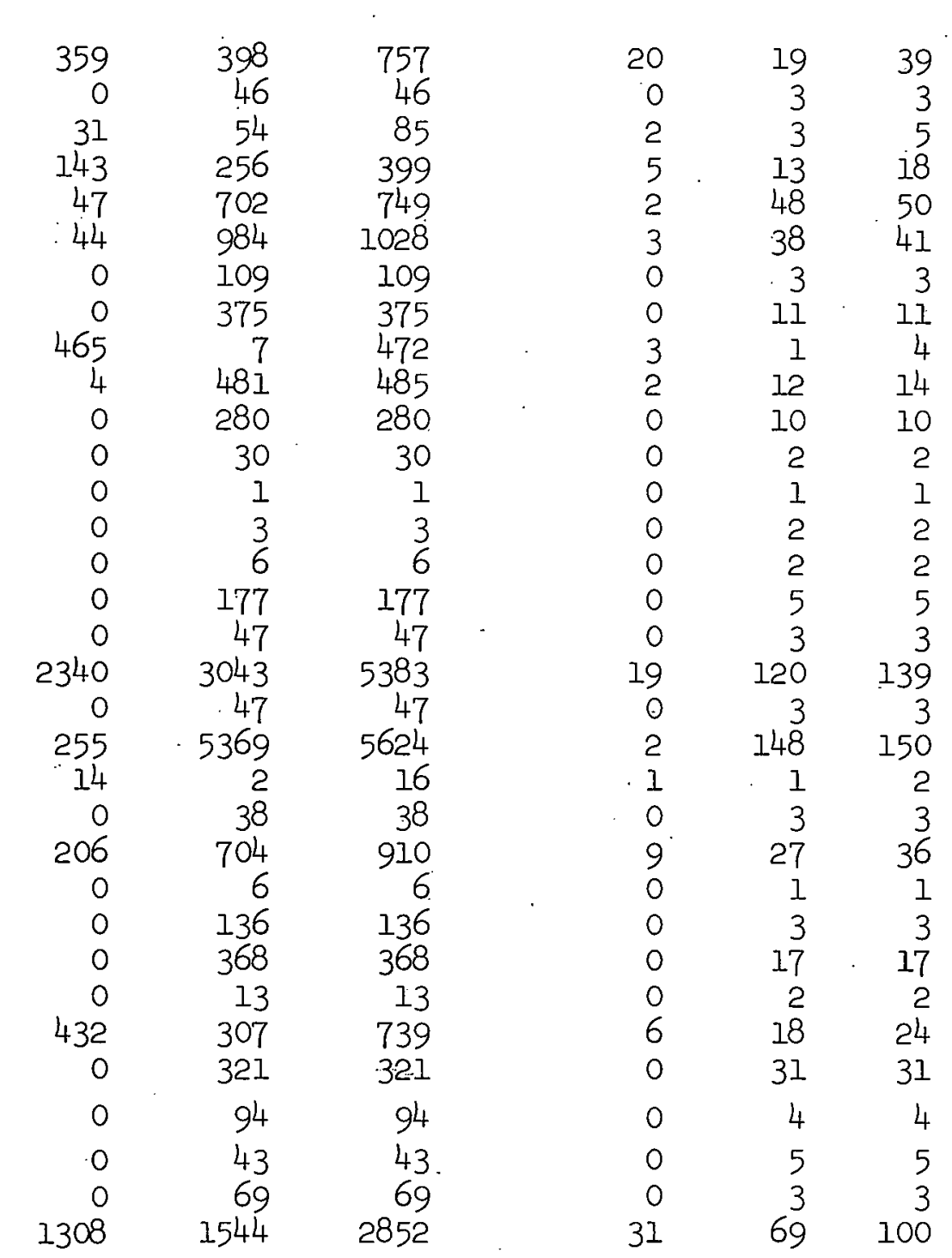

$\mathrm{T}$ and $\mathrm{E}^{2}$

Res

Total

ACCY

$A G E C$

ANGEN

ANTH

ASTR

BEDRE

CCDME

CCENE

$\mathrm{CE}$

CERE

CP

DCS

DS

DCON

ED

EDADM

EDTES

$\mathrm{EE}$

1308

1544

100

253.

0.0
48.0
47.4
11.0
18.7
0.0

0.0

628.7

0.5

0.0

0.0

0.0

0.0

0.0

0.0

0.0

2941.2

0.0

$2 \cdot 21.8$

11.6
0.0

317.8

0.0

0.0

0.0

0.0

325.4

0.0

0.0

0.0

0.0

\begin{tabular}{ll}
807.7 \\
\hline
\end{tabular}
9. $29.7 \quad 1223.2$ $31.3 \quad 31.3$

$106.5 \quad 154.5$

.316 .644 .0

$\begin{array}{llll}83 & 33.8 & 83 & 44.8\end{array}$

2133.62152 .3

$23.2 \quad 23.2$

$\begin{array}{lll}658.7 & 658.7\end{array}$

$8.4 \quad 6 \quad 37.1$

$\begin{array}{llll}4 & 1.2 & 4 & 1.7\end{array}$

$\begin{array}{lll}2 & 37.4 & 2 \\ 56.4 & \end{array}$

$56.1 \quad 56.1$

$0.6 \quad 0.6$

$2.0 \cdot 2.0$

4.6

$643.9 \quad 643.9$

$14.8 \quad 14.8$

112. $34.6 \quad 142 \quad 15.8$

$12.3 \quad 12.3$

$13018.4 \quad 13240.2$

9.521 .1

118.2118 .2

$1430.1 \quad 1747.9$ $5.0 \quad 5.0$

$102.5 \quad 102.5$

246.5246 .5

$13.8 \quad 13.8$

606.6932 .0

$855.5 \quad 855.5$

$217.9 \quad 217.9$

$137.1 \quad 137.1$

$44.9 \quad 44.9$

$\begin{array}{lll}19 & 26.1 & 27 \\ 33.8\end{array}$ 
Quarterly Summary of Departmental Running Time April, May, June, 1966 Continued

\begin{tabular}{|c|c|c|c|c|}
\hline & Dept $^{1}$ & & $r$ of $R$ & \\
\hline & & $T$ and $E^{2}$ & $\operatorname{Res}$ & Total \\
\hline & ENTOM & O & 11 & 11 \\
\hline & FIN & 86 & 18 & 104 \\
\hline & FOR & 0 & 42 & 42 \\
\hline & $\mathrm{FT}$ & 0 & 10 & 10 \\
\hline & GENE & $280^{\circ}$ & 50 & 330 \\
\hline & GEOG & 14 & 10 & 24 \\
\hline & GEOL & 0 & 119 & 119 \\
\hline & GSBA & 239 & 59 & 298 \\
\hline & HEC & 0 & 1 & 1 \\
\hline & HED & 0 & 17 & 17 \\
\hline & HLTHS & 0 & 2 & 2 \\
\hline & HONOR & 0 & 4 & 4 \\
\hline & HORT & 0 & 99 & 99 \\
\hline & ICR & 0 & 427 & 427 \\
\hline & IE & 504 & 27 & 531 \\
\hline & IEED & 0 & 25 & 25 \\
\hline & IGPA & 0 & 97 & 97 \\
\hline & $\Pi \mathrm{LR}$ & 0 & 35 & 35 \\
\hline & INADM & 237 & 6 & 243 \\
\hline & IREC & 0 & 103 & 103 \\
\hline & LIR & 0 & 31 & 31 \\
\hline & MATH & 3237 & 2 & 3239 \\
\hline & MATRL & 0 & 1801 & 1801 \\
\hline & MCBIO & 0 & 44 & 44 \\
\hline & $\mathrm{ME}$ & 1668 & 1669 & 3337 \\
\hline & MMPE & 0 & 603 & 603 \\
\hline & MUSIC & 0 & 48 & 48 \\
\hline & NHS & 0 & 169 & 169 \\
\hline & NUCE & 462 & 582 & 1044 \\
\hline & OIR & 0 & 828 & 828 \\
\hline & PEM & 0 & 99 & 99 \\
\hline & PEW & 0 & 7 & 7 \\
\hline & PHYCS & 2 & 10101 & 10103 \\
\hline & PHYSL & 0 & 18 & 18 \\
\hline & PLPA & 0 & 7 & 7 \\
\hline
\end{tabular}

Number of Specs $\mathrm{T}$ and $\mathrm{E}^{2}$ Res Total

$\begin{array}{rrr}0 & 2 & 2 \\ 3 & 3 & 6 \\ 0 & 8 & 8 \\ 0 & 3 & 3 \\ 6 & 3 & 9 \\ 2 & 1 & 3 \\ 0 & 6 & 6 \\ 7 & 2 & 9 \\ 0 & 1 & 1 \\ 0 & 4 & 4 \\ 0 & 1 & 1 \\ 0 & 1 & 1 \\ 0 & 11 & 11 \\ 0 & 7 & 7 \\ 9 & 3 & 12 \\ 0 & 2 & 2 \\ 0 & 4 & 4 \\ 0 & 2 & 2 \\ 6 & 3 & 9 \\ 0 & 10 & 10 \\ 0 & 3 & 3 \\ 7 & 1 & 8 \\ 0 & 37 & 37 \\ 0 & 2 & 2 \\ 33 & 76 & 109 \\ 0 & 21 & 21 \\ 0 & 3 & 3 \\ 0 & 6 & 6 \\ 13 & 18 & 31 \\ 0 & 3 & 3 \\ 0 & 11 & 11 \\ 0 & 2 & 2 \\ 1 & 129 & 130 \\ 0 & 3 & 3 \\ 0 & 1 & 1 \\ 0 & & \end{array}$

7094 Usage in Hours-Minutes $\mathrm{T}$ and $\mathrm{E}^{2} \quad \operatorname{Res}$ Total

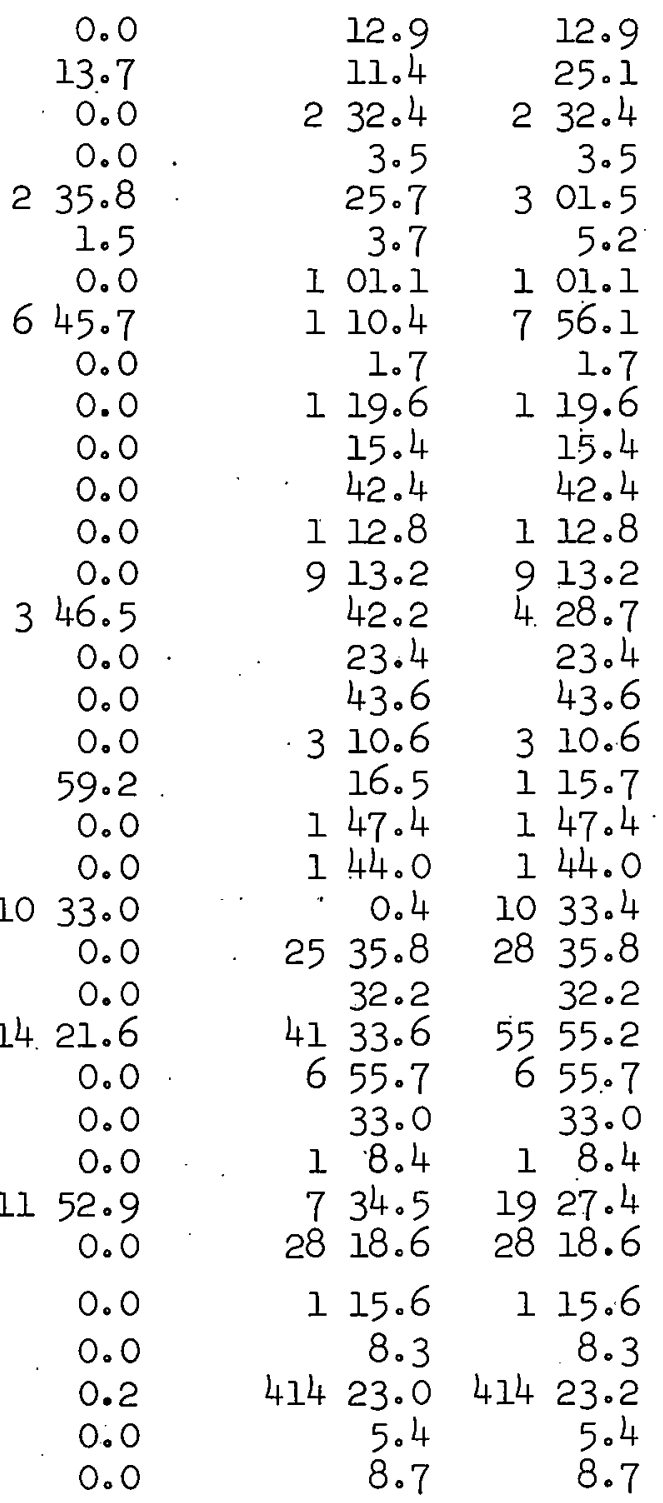


Quarterly Summary of Departmental Running Time

April, May, June, 1966 Continued

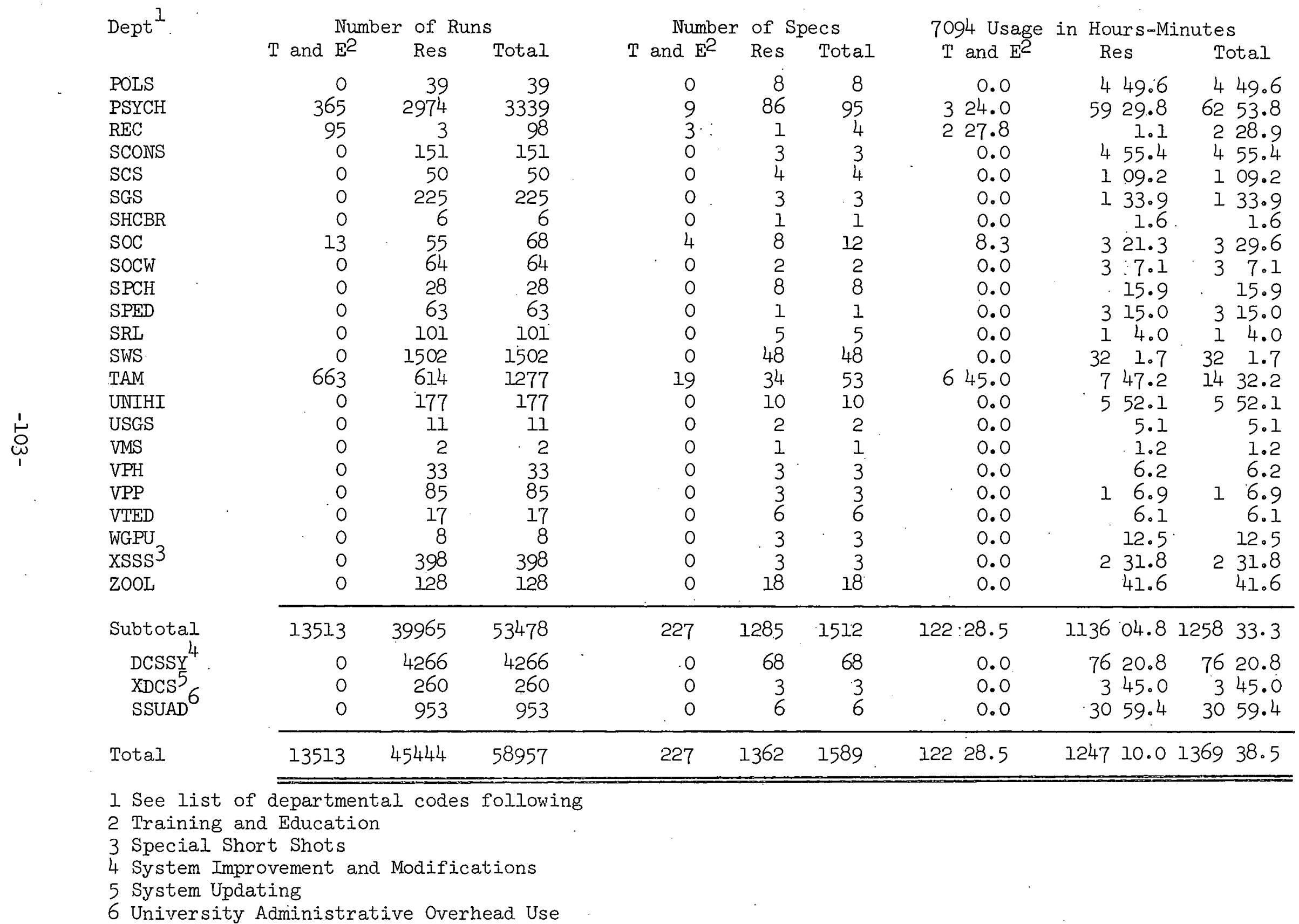




\section{LIST OF DEPARTMENT' CODES}

IF YOUR DEPARTMENT OR OFFICE DOES NOT APPEAR ON THIS LIST, PLEASE WRITE ITS FULL NAME IN THE DEPARTMENT FIELD (B) ON THE PROBLEM SPECIFICATION FORM EVEN THOUGH IT WILL REQUIRE MORE THAN 6

ACCY CHARACTERS.
ADMREC

ADV

$A A E$

AGEC

AGED

$A G E$

AGREXT

$A G R$

AGRON

ANS

ANTH

$A R C H$

$A R T$

ASTR

BIOPH

BOT

$B C M P L$

BECBSR

BEDRES

GSBA

$C Z R$

CERE

CHE

CRC

$C P$

$C E$

CCARCH COMM

CSL

DS

DT

DNSTUD

DCS

DGS

DUE

ECON

ED

EDPSY

EDADM

EDTEST

EE
ACCOUNTANCY

ADMISSIONS AND RECORDS

ADVERTISING

AERONAUTICAL AND ASTRONAUTICAL ENGINEERING

AGRICULTURAL ECONOMICS

AGRICULTURAL EDUCATION

AGRICULTURAL ENGINEERING

AGRICULTURAL EXTENSION

AGR I CULTURE

AGRONOMY

ANIMAL SCIENCE

ANTHROPOLOGY

ARCHITECTURE

ART

ASTRONOMY

BIOPHYSICS

BOTANY

BUREAU OF COMMUNITY PLANNING

BUREAU OF ECONOMIC AND BUSINESS RESEARCH

BUREAU OF EDUCATIONAL RESEARCH

BUSINESS ADMINISTRATION, GRADUATE SCHOOL

CENTER FOR ZOONOSES : RESEARCH

CERAMIC ENGINEERING

CHEMISTRY AND CHEMICAL ENGINEERING

CHILDREN'S RESEARCH CENTER

CITY PLANNING

CIVIL ENGINEERING

ARCHITECTURE (CHICAGO CIRCLE)

COMMUNICATIONS

COORDINATED SCIENCE LABORATORY

DAIRY . SCIENCE

DAIRY TECHNOLOGY

DEAN OF STUDENTS

DEPARTMENT OF COMPUTER SCIENCE.

DIVISION OF GENERAL STUDIES $L A S$

DIVISION OF UNIVERSITY EXTENSION

ECONOMICS

EDUCATION

EDUCATION PSYCHOLOGY.

EOUCATIONAL ADMINISTRATION AND SUPERVISION

EDUCATIONAL TESTING

ELECTRICAL ENGINEERING 


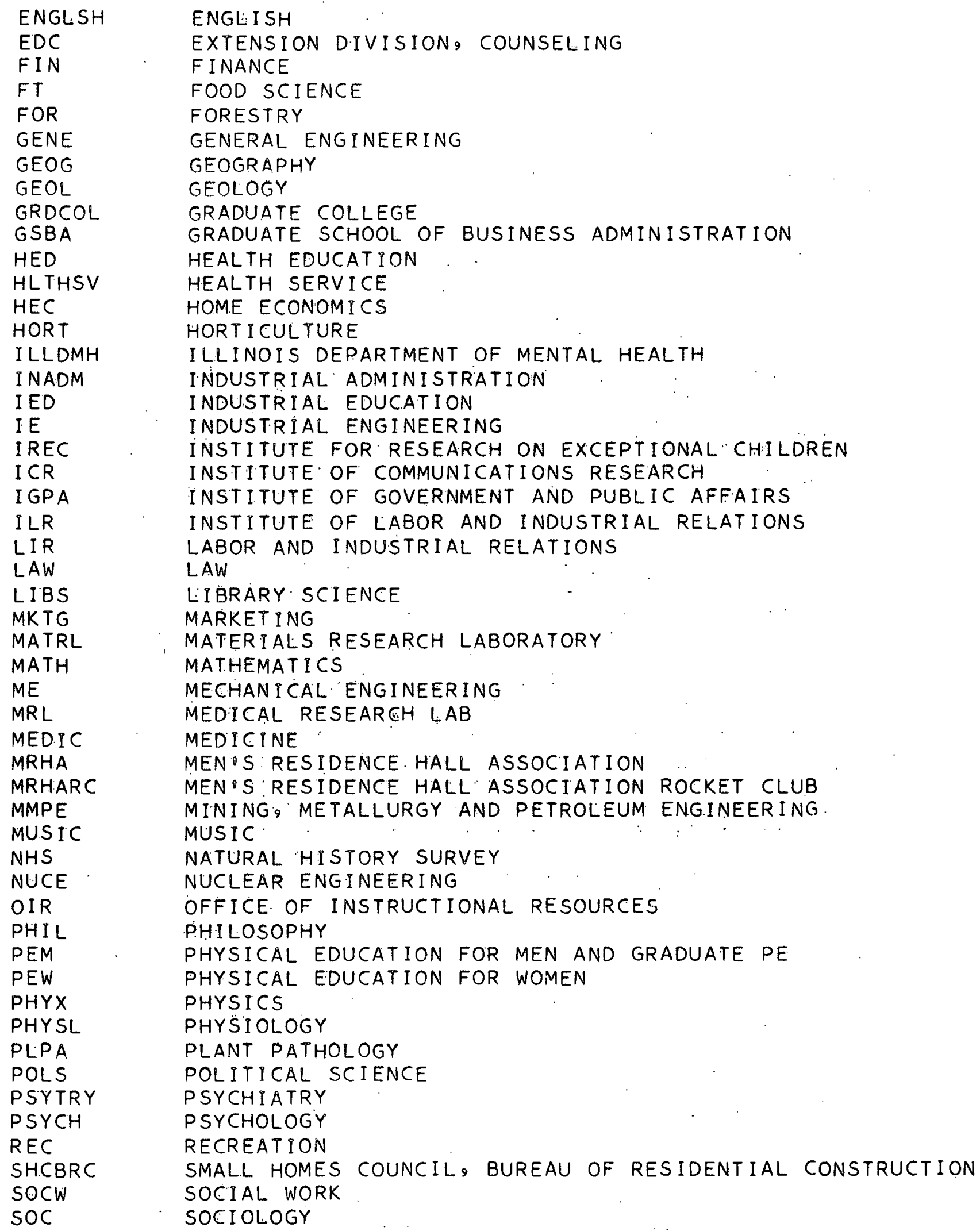




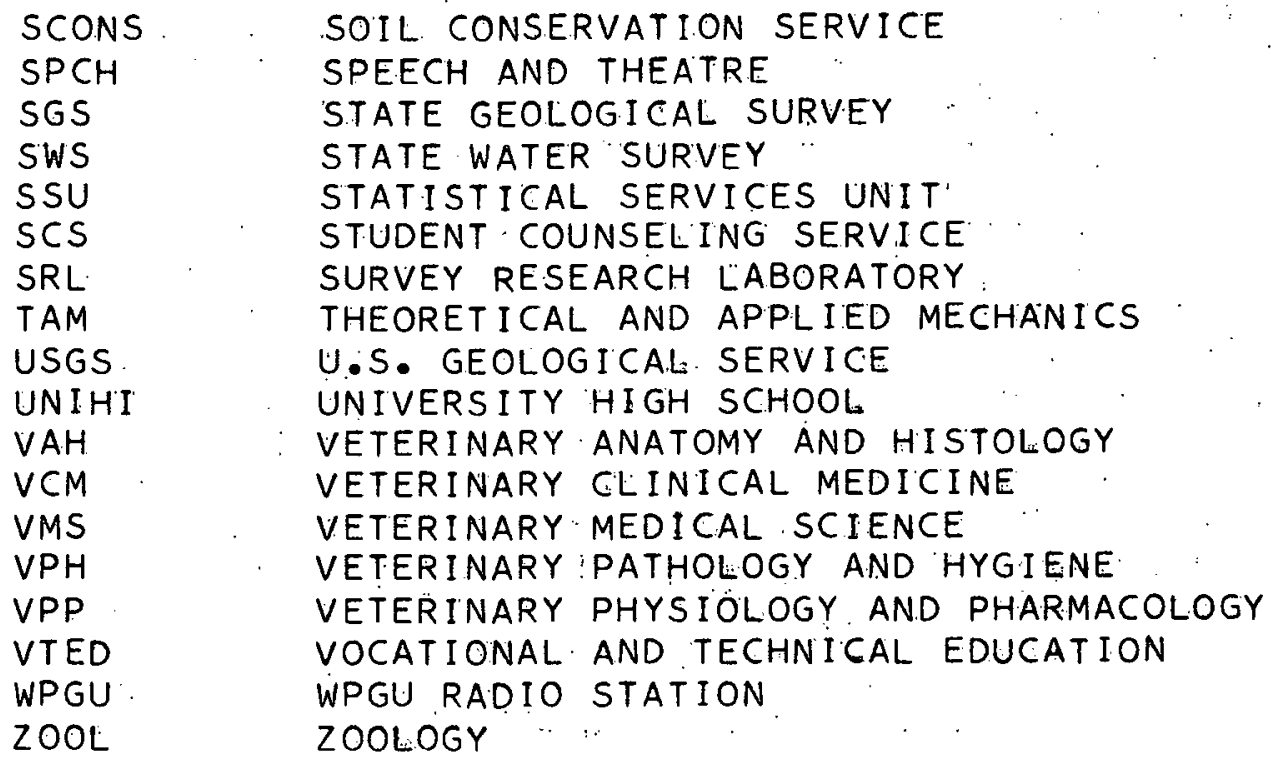

$-106-$ 


\section{PROBLEM SPECIFICATIONS}

(Supported in part by the National Science Foundation under Grant No. NSF-GP-700.)

\subsection{Research Problem Specifications}

During the second quarter of 1966, 154 problem specifications were submitted to the Department for computation. The following brief descriptions of these problems have been prepared for inclusion in this report by those submitting them. T indicates a calculation associated with a thesis.

1947-64001 T Psychology. The Parameters of Cross-Validation. The crossvalidation method of estimating multiple correlations from sample values will be investigated by generating artificially on the computer data from the multivariate normal distribution. A number of criterion variables as well as predictor variables will be generated. Several parameters of the joint distribution will be systematically varied. The effect of these parameters on the estimation of the multiple correlation and on the determination of the number of criterion relevant dimensions in the predictor space will be studied. (Paul Herzberg)

1948-64003 T Eclucation. Teacher-Pupil Relationship as Related to Selfconcept and Achievement Motivation. Pearson product moment correlations will be obtained to assess the value of the relationship between the three variables stipulated in the problem title. These correlation values will be subsequently used to obtain a multiple correlation value for "teacherpupil relationship" as it is related" to self concept and achievement motivation. Both Pearson $r^{\prime}$ s and multiple correlation values will be obtained on total and sub-group samples. In addition to correlation values, t tests for independent sub-groups will be taken to assess the significance of differences of mean values. (Gerald M. Knowles)

1949-64004 T Education. Teacher Influence on 4th Grade Composition. This study is attempting to assess the influence of the teacher on the composition of 4 th grade pupils. Such variables as teacher writing, ability as measured by the Step Essay Test, and the pupil perception of "The Teacher", "The Classroom", "The Assignments" and "The Discussions" 
as measured by a modification of the Osgood Semantic Differential are related to the mean scores of the pupils'writings which were also measured by the Step Essay Test. Both group and individual analysis will be made。 $t$ tests will be used to assess the differences of the means of the groups. Twelve classrooms are being used in this study. Six of the teachers scored high on the Step Essay Test (Form la) and six scored low. The students were given Form $4 a$ of the same type of essay test. (Richard Biberstine)

1950-64005 T Psychology. The Empirical Demonstration of Some Relationships in Differential Prediction. The research involves the empirical demonstration of several mathematically derived relationships concerning moderator variables and indices of predictibility in prediction situations: (1), the use of suppressor variables as indices of predictibility; (2) the effect of having extraneous non-variable traits in standardization groups upon prediction in groups with the same, but more variable traits and correcting for this by the use of moderators; (3) the relationship between indices of predictibility and moderators; and (4) the effect of moderators and indices of predictibility in multiple regression. These analyses will be done on the Parental Attitude and Behavior Inventory and Teacher's Behavior Checklist. In addition to the usual scales of these questionnaires, special scales will be developed. The data were collected on approximately 700 subjects, divided equally between parents of children who do not attend a mental health clinic and of children who do attend a mental health clinic. The teachers ratings were done by the teachers of these children. The children are in the first to sixth grade inclusive. The parents are matched on various background variables (age, education, socioeconomic standing, etc.) (Conger)

1951-64006 T Educational Administration and Supervision. A Comparison of Teachers' and Administrators: Perceptions of Decision Making. The purpose of this study is to determine where differences, if any, exist between teacher and administrator perceptions of the existing and ideal pattern of decision-making in selected elementary school districts in the state of Illinois. Four hypotheses will be tested: 1. There are 
statistically significant differences between teachers ' and administrators' perceptions of existing patterns of decision-making. 2. There are statistically significant differences between teachers' and administrators' perceptions of the ideal patterns of decision-making. $\dot{3}$. There are statistically significant differences between the teacher's' perceptions of the existing and ideal patterns of decision-making. 4. There are. statistically significant differences between the administrators' perceptions of the existing and ideal patterns of decision-making. Hypotheses one and. two will be tested using a t test for difference of means of each cell for independent groups. Hypotheses three and four will be tested using a $t$ test for difference of means of each cell for paired groups. (Thomas E. Benner Jr.)

1952-64008 T Chemistry and Chemical Engineering. Nuclear Magnetic Resonance Anisotropy Dipole Lineshape Moments. The problem involves the calculation of line shapes for solid state nuclear magnetic resonance. The analysis will apply to nuclear spin subsystems, whose energy is orientation dependent, isotropically distributed. Since the effect of isotropic distributions is difficult to determine analytically for all but very elementary systems of spins, a numerical method is proposed in which the orientations of the subsystem are allowed to vary. One then calculates the energy for each orientation, thereby getting the line shape. After the line shape is obtained, the first and second moments of the lines will also be calculated; however, this will be a relatively simple matter. For more complicated systems, a matrix diagonalization will probably precede the calculation.

(David L. Vanderhart)

1953-64009 State Water Survey. Time Cross-Sections Cloud and Weather. Hourly weather messages and special reports from twenty airfields form the basic observational data from which time-cross-sections of wind direction, wind speed, cloud cover and cloud base heights will be plotted. The trend of these variables and discontinuities in the trend will give information about the onset and development of important physical processes in the atmosphere. 
The computer program will sort the data with respect to station number and the times will be put in order. The cloud base height will be categorized into low. level and medium or high level clouds and the code in which the data are received will be converted into standard units (hours and decimals, meters, etc.) (Pieter Feteris)

1954-64010 T Electrical Engineering.:. Optimal Motor Control. The problem to be studied is the design of an "optimal" control system for motors in' a traction application. MIDAS will be used to aid in the design and evaluation of this nonlinear control system. MIDAS is a digital computer technique which has been developed to simulate a set of differential equations or other systems much in the same way as an analog computer does. Thus the desired system will be simulated and changes in the system performance; resulting from parameter variations will be observed. Because of the intrinsic nonlinearities in the system a mathematical solution of this problem is impossible and system performance can only be obtained by simulation. (Richard J. Stec)

1955-64011 Department of Computer Science. Music Composition and Plotting。 This program will plot the elements of a musical score in a way which will allow the plot to be converted to player piano roll. This procedure will allow the "playing" of piano compositions which are technically impossible for human pianists. Source of the musical data will be Hilleis Musicomp program. (J。L. Divilbiss)

1956-64013 Speech and Theatre. The Effect of Progressive Physical Training on the Reduction of Stuttering. The purpose of this study is to determine the effects of a four month progressive physical training program on the reduction of the level of severity of stuttering in students currently enrolled in speech therapy. The subjects are eight students currently enrolled in speech therapy at the University of Illinois Speech Clinic. Four of the students are in group one, the experimental group, which is having therapy plus the progressive physical training program. The 
remaining four students comprise the control group, which is having only stuttering therapy. There will be two testing periods, at which the students will be administered tests to determine the level of the severity of stuttering and also the level of physical fitness. The data will be analyzed by computing a t-test for the difference between means on each of the stuttering and physical fitness test items (approximately 45) to determine any significant differences (at the .05 and .01 levels) between the groups at the first and last testing periods and within the groups between the two testing periods. (Elizabeth B. Franks)

1957-64014 Civil Engineering. Locomotive. Assignment Simulation. The object of this project is to study the effect on operations of assigning different types of locomotives to railroad trains. Use will be made of the Train Performance Simulator program developed in the Civil Engineering Systems Laboratory for evaluating effects on over the road train performance. The problem to be attacked is that of a high-speed merchandise freight train which, because of numerous speed restrictions, does not fully utilize the motive power assigned to it. By means of the Train Performance Simulator program, various motive power combinations will be tried on the train. For each type of locomotive the savings in ownership and operating costs as well as the effect of longer "over the road" times and slower service will be evaluated.

When this stage of the program is complete, investigation will begin into the effect of increasing the restrictive speed limits. For each new speed limit all motive power configurations will be simulated. Once again it will be possible to weigh the increased operating and maintenance cost due to higher speed operations with the benefits gained by shorter "over the road" times and faster service.

When all these simulations have been performed it will be possible to contrast the advantages and disadvantages of each type of operation and determine what combination of locomotive and speed limit is the optimum for this type of freight train operation. (R. W. Drucker) 
1958-64015 Physics. Optical Absorbtion of Weak Binding Exciton in a Magnetic Field. The data from experiments on exciton optical absorbtion and magneto absorbtion in thallium chloride and thallium bromide will be evaluated. Typical procedures contemplated will involve curve fitting and Kramers Kronig dispersion relation analysis to evaluate the real and imaginary part of the dielectric constant. (Robert Bachrach)

1959-64016 Educational Psychology. Teaching. Pitch Discrimination. Recent research with visual stimuli and phonemes shows that by sequencing helpful cues or "prompts", fine discriminations can be taught to young children with very low error rates. This research project will extend this work to pitch discrimination. One objective of this project is the development of an instructional program that will teach fine pitch discrimination to young children. The development of such an instructional program requires precise control of the stimuli. This means exact control of the pitch frequencies of pure tones, the intensity of the tones, and the time intervals used throughout the teaching sequence. A computer program prepared for the production of electronic music provides the necessary control of the variables mentioned above. The tones, as produced by the computer, will be recorded and presented to the students by means of a tape recorder. (R. C. Anderson)

1960-64017 T Chicago Circle Department of Energy Engineering. Nonlinear Integral Equations for Heat Transfer. This problem concerns calculations of temperature profiles in Coetle flow with a medium of variable absorption coefficients. The case consists of the interaction of radiation upon other modes of heat transfer, such as conduction and convection. The successive iterations method is used for solution of the nonlinear integral equation concerned. The results will be used to calculate the heat transfer and compare it with other cases where radiation medium has a constant absorption coefficient. (Ramadan) 
1961-64018 T Educational Psychology. Role and Function of Elementary School Counselors as Perceived by Counselors, Teachers and Administrators. Twenty.. demonstration pilot centers are to be studied. Twenty counselors will be given pre and post tests with a sixty item forced choice seven category $Q$-sort instrument. Approximately three hundred and fifty teachers and twenty administrators at these twenty centers. will also use this Q-sort instrument. Approximately ten to fifteen faculty (counselor/educators) will use the Q-sort instrument to set. up a model. A Pearson " $r$ " correlation, factor analysis, and analysis of variance will be computed. Comparisons will then be made between counselors, teachers, and administrators for change of attitudes and for dissonance (changes over the year). Comparisons will also be made between centers and between counselors in centers. with administrators and with teachers. (Glofka. Petert)

1962-64019. Civil Engineering. Torsional Stress Concentration. The problem to be solved is the evaluation of the stress concentration arising from structural discontinuities in a cylindrical shaft subjected to torsion. The computer is to be used for the solution of the simultaneous equations resulting from the finite difference expressions for the governing equation. These results will then be used for determination of stresses throughout the shaft. (Lance. Boichot)

1963-64022 Civil Engineering. Discrete Models of Nonlinear Solid Media. Discrete models applicable to broad classes of problems of nonlinear solid media are developed. Specific areas of investigation include wave propagation in elastic-perfectly plastic media, generalized CoulombMohr solids, and soil type solids; quasi-statical problems of bulk structures such as plates under small and large deflections are also being studied. Dynamical problems involving large density changes, such as those occurring in earth materials, are considered from the general conservation standpoint. (A. Ang) 
1964-64023 T Agricultural Economics. Estate Management. This research problem will involve linear programming techniques. An objective function will be maximized or minimized when subject to linear inequalities or constraints: Models used will be solvable by the simplex algorithm. The LP/90 operating system is appropriate for solution of this problem because of the large dimensions of the programming model to be used. (Gerald A. Harrison)

1965-64024 T Materials Research Laboratory. Double Integration of Experimental Curves. It is necessary to evaluate the second integrals of experimental curves which arise in a nuclear magnetic resonance experiment. The curves are obtained in the form of a voltage varying in time, and are to be converted into digital form by a digital voltmeter which incorporates provision for printing its output. (G. W. Stupian)

1966-64025 T Electrical Engineering. Saturation Theory and Noise in Field Effect Transistors. In this project, it is required to solve several equations obtained from theoretical analysis by employing numerical computations. The first part is to determine the saturation drain characteristics of the junction gate field effect transistors, for which seven simultaneous equations with seven unknowns must be solved. The second part is to calculate the equivalent input noise resistance. The results of the first part will be applied to this calculation. Double integrals will be calculated by using Simpson's rule and the UITR2 library subroutine. The data are obtained from theoretical analysis. The computer results will be graphed in several figures and tables which will be used in the thesis. (S. Y。Wu)

1967-64026 University High School. Factors in Evaluation of Demonstration Centers. Questionnaires for the purpose of analyzing people's views on children of various talents and curriculum development in math, science, English, and social studies were and will be distributed to educators 
and parents throughout Illinois. The results should provide information to the Department of Program Development for Gifted Children about the effectiveness of Demonstration Centers in Illinois. The following will be conducted: (i) tabulation of results of experimental instruments (item means, factor analysis, factor means); (ii) comparison of pre-test with post-test data by a t-test of means and analysis of variance; and (iii) comparison of pre-test with post-test by chi-square. (W. M. Rogge)

1968-64027 T Civil Engineering. A Study of Rainfall Excess-Surface Run Off. The storage in a basin has been conclusively demonstrated to be dependent on both the inflow and outflow characteristics. Also the hydrograph correlation seems to be better if the input is taken into consideration. In a previous analysis, the reproduction of runoff data for various storms considered is good up to the peak of the hydrograph. The recession portions of the hydrographs are not so closely reproduced. A possible: reason for this occurrence may be that terms to consider the effect of input are not included. Hence, it is felt that in any analysis of hydrologic systems both the inflow and outflow should be considered as affecting the storage characteristics of the basin. The effect of the inflow may be small, and is zero after the cessation of rainfall. However, it should be included in the analysis.

In the first part of the analysis, the data to be used will be taken from the experiments to be conducted using the rainfall simulator available in the Civil Engineering Department. The data available from the simulator are specially desirable, due to the accuracy with which it can be measured and controlled. In the second part of the analysis field data are to be used. (A. R. Rao)

1969-64028 T Electrical Engineering. Scattering of Ultrasound by Rigid Particles. A number of theories exist which predict the values of absorption coefficients of ultrasound in suspensions of spherical particles. The computer will be used to calculate these values from the physical parameters of suspensions. These values will then be compared to the experimental data in order to determine applicability of each theory. (S. A. Hawley) 
1970-64029: Finance. Banking Concentration Study. This is a study of the economic effects of banking concentration. The computer will compute ratios of banking concentration for each county in the 7 th Federal Reserve District. The ratios to be computed are: (a) percent of county assets held by the two largest banks; (b) percent of county assets held by the largest. $50 \%$ of the banks; (c) number of banks holding $50 \%$ of county assets. (A. J. Heins)

1971-64030 T Educational Psychology。Analysis of Faculty-Student Relations Index. A sample of students from a number of public junior colleges have responded to an instrument titled the Faculty-Student Relations Index. This instrument contains 144 items grouped in 16 subtests or scales of varying: length. The item responses will be scored for each individual on each subtest and a profile of scale or subtest: scores obtained for each college. The colleges will be grouped on variables hypothesized to be related to faculty-student relations and an analysis of variance used to test these hypotheses. The groups' time tests interaction will be used as a means of testing profile differences. (William Wellner)

1972-64031 Accountancy. Business Simulation Problems. The purpose of this project is to investigate the applicability of three computer languages,; FORTRAN, SIMSCRIPI, and GPSS-III, for simulations of accounting related situations. This phase of the project will consist largely of familiarization with the latter two languages. The data to be used in the project will be hypothetical. It. will be designed to test and compare specific characteristics of the languages. It is hoped that the project will lead to a new approach to teaching problem solving related to annuity problems.. (Mike Uretsky)

1973-64032 T Mining, Metallurgy, and Petroleum Engineering. Moving Interface. It is desired to find the motion of the interface between two different fluids. The motion of the fluids is described by the Navier Stakes equation. The interface will be subject to the constraint of minimum entropy production. The equation is solved for two dimensional flow using an iterative technique. (B. Singh) 
1974-64045 T Psychology. Personality and Social Attitudes Research. The purpose of this study is to see how subjects of differing personality respond to other individuals whose personality and race are specified by the experimenter. The personality of the subjects was measured with a standard personality test and their behavioral intentions were measured with the Behavioral Differential. The Behavioral Differential is a questionnaire designed to measure interpersonal behavioral intentions toward various persons described to the subjects. Behavorial intentions are reported on fifteen nine-point bi-polar scales. One pole represents the response of a definite intention to do the act specified on the scale with the person described. The other pole represents the response of a definite intention not to act in the manner specified. Gradations between extreme intentions are represented by the more central points on the scales. Sixteen persons were described on four facets: Negro-white; extrovert-introvert; agreeable-nasty; and. "A" student-"C" student. Ninety-seven subjects were used. Behavioral intention factors will be found by factor analysis of the nine-point scales. A $2 \times 2 \times 2 \times 2$ analysis of variance will be done on the composite scores of the 16 hypothetical persons on the obtained factors. The analyses of variance will indicate the relative importance of the various facets of the descriptions of these persons in determining the behavioral intentions of the subjects. The Tucker-Messick two mode factor analysis with varimax rotation and counter rotation of the variable mode will also be performed on the data. These subject factors thus obtained will be correlated with the subjects personality scores and the matrix of intercorrelations factor analyzed. It is hypothesized that certain of the subject factors obtained from the Tucker-Messick procedure will be systematically related to the personality characteristics obtained from the standard personality test, thus lending support to the construct validity of both measures. (Posavac) 
1975-64044 Ceramic Engineering。 Computation of Crystalline D-Spacings . This problem is concerned with an investigation of the crystalline unit cell parameters of several compounds which crystallize with the perovskite structure. These compounds often exhibit multiplicity in their structures, i.e., several simple perovskite blocks are required to properly describe the true crystalline unit cell. Due to this multiplicity and the resulting large size of the unit cell, it is very difficult to index the lines occurring on an $x$-ray powder diffraction pattern of this type. This step is necessary in order to be able to establish the unit cell parameters. The parameters of the perovskite blocks composing the structure can be accurately measured. With this information and an estimate of the parameters one can index the patterns if all of the possible $x$-ray reflections can be computed and compared with the measured values. The computer will be utilized to calculate all of the possible $x$-ray reflections up to the 15th order for crystals having cubic, tetragonal, orthorhombic, and monoclinic symmetry in which the parameters are estimated from the experimental diffraction patterns. (V. Tennery)

1976-64042 Agricultural Economics。 Factor Analysis Regression. Factor analysis regression will be used to estimate various empirical relationships. These estimates will be compared with least squares regressions. The distribution functions of the residuals from factor analysis regression will be studied in order to develop test statistics for factor analysis regression. Factor analysis, matrix manipulation, distribution analysis, and regression programs. will be used. Most of the data to be used are census time series and cross sectional data. Results will be used for basic development of models for economic analysis where there are errors in the variables. Also, such models are applicable in other disciplines where there are errors in the variables. (J.T. Scott) 
1977-64041 T. Political Science. Societal and Conflict Dimensions in International Relations. The problem under study concerns the relationship between the similarity of societal-structural characteristics of 608 pairs of nations and the conflict occuring between the members of the pairs. Seventy-one variables are used to describe each pair of nations in terms of its differences in socioeconomic and political characteristics. Aspects of conflict between nations are depicted by thirteen variables. The computer will be used to cross-classify the societal and conflict variables and to calculate measures of association among the variables. (Leila Fraser)

1978-64040 Agricultural Economics. Effect of Industrialization on Farm Organization. This project will estimate the effect of local industrialization on the use of factors, products produced, and organization for various sizes and types of farms. Special emphasis will be made on land use, labor use, and grain and livestock production within the overall production and marketing agricultural sector of a five county Illinois area: LaSalle, Bureau, Marshall, Putnam, and Stark. Data. will be obtained through surveys, detailed cost studies, and census data. Linear programming will be used as well as. regression and factor analysis. Results will be useful in advising other communities and making predictions in other areas of industrial impact. (J.T. Scott)

1979-64039 T Health Education. A Statistical Analysis of the Huffman Inventory. The purpose of the study. is to devise a paper-pencil inventory which can measure emotional immaturity effectively. A preliminary instrument, the Huffman Inventory, was constructed and administered to 1233 subjects. Test data from these subjects were transcribed on Digitek forms. There were seven sub-groups of subjects, as follows: (1) 175 male teachers; (2) 46 female teachers; (3) 185 adult male non-teachers; (4) 198 adult female non-teachers; (5) 318 high school male students; (6) 247 high school female students; and (7) 64 college male students. Cards were punched from the Digitek form in preparation for statistical analysis by the computer. 
Standard mathematical methods will be used to analyze the data for the total sample, each sub-group, and among the sub-groups. The inventory will then be revised according to the statistical results and administered to new subjects. A final statistical analysis by the computer will then be made. (Nancy J. Coble)

1980-64038 Health Education. Survey of Institutions Offering Professional Preparation in Health Education. The study is designed to determine the nature and extent of professional degree programs in health education at the master's level in the colleges and universities of the United States. The population surveyed included all colleges and universities offering programs, except those offered in Schools of Public Health. The questionnaire method. was used in the collection of data for the study. The respondent sought in each case was the person responsible for the master's degree program in each of the 50 institutions listed as having master's degree programs in health education. A 96 per cent response was achieved. The SSUPAC collection. of programs will be used to calculate cross tabulation for pairs of variables and measures of association from cross classification tables. The results of the study are to be used to provide a baseline in restudying the functions and the preparation of health educators. It is anticipated that standards will be formulated which will eventually relate to accreditation of such programs of preparation. (W. H. Creswell, Jr.)

1981-64037 Survey Research Laboratory. Data Storage and. Retrieval. The data involved are survey data from many sûrveys previously taken on various subjects such as the 1964 United States elections and opinions on controversial topics. The data are presently on cards, and axe to be placed. on tape for easier handling. These data are used when the Survey Research Laboratory is asked to do some secondary analysis by a client. who wants to analyze the data gathered from a survey conducted in past years. (P.S. Schuman) 
1982-64036 . Civil Engineering. Structural Optimization. The problem of obtaining the least-weight elastic design of a steel structural frame can be formulated as a problem in nonlinear mathematical programming. The resulting programming problem can be solved using existing steepest-descent algorithms. The purposes of the proposed research are the following: (1) to develop methods and computer programs for obtaining least-cost designs; (2) to develop improvements in the program for solving the programming problem, (a) by comparing the efficiency of the various programming algorithms, and (b) by developing more efficient means of calculating the derivatives of the constraint functions; (3) to develop a problem-oriented-language computer program for obtaining least-cost structural designs. (D. M. Brown)

1983-64035 T Mechanical Engineering. Statistical Behavior of Small Particles in Turbulent Flow Field. An analysis is made of the statistics which govern the turbulent transport behavior of small particles. The equation of particle motion is transformed such that the non-stationary particle velocity fluctuations are sent to ones with stationary fluctuations. The transformed integro-differential equation is then treated as a stochastic equation to which the Fourier analysis is applied. Expressions are obtained which relate the Lagrangian energy spectrum of the particle to the Eulerian spectrum of the fluid. Experimentally, the Eulerian spectrum is obtained from hot-wire measurements and the Lagrangian spectrum by following the particle motion continuously. The voltage signals in both cases are recorded directly on magnetic tapes, and are then digitized at Argonne National Laboratory. The digitized raw data is then reduced to obtain. the above spectrums for the experimental verification of the analysis. (Mostafa Shirazi)

1984-64034 T Education. The Predictive Validity and Stability of the Illinois Test. of Psycholinguistic Abilities. The study will attempt to assess the ability of the Illinois Test of Psycholinguistic Abilities (ITPA), when given at the beginning of kindergarten, to predict academic achievement at the beginning of second grade, as indicated by the 
California Achievement Tests. A second purpose is to test the stability of the ITPA over a span of two years. The sample consists of 72 children from the Urbana Public Schools. The design will be primarily correlational, making use of step-wise multiple correlation. (Alfred Hirshoren)

1985-64046 T Psychology. Comparison of Behavior and Attitude Change Techniques. Five groups of twenty subjects each will be compared over three time intervals on several motivational variables and sixteen personality variables. There will be two kinds of analyses: 1) analyses of covariance and 2) Pearson product moment correlations. (Maurath)

1986-64047 Natural History Survey• Cottontail Rabbit Research. The problem consists of two unrelated parts. First is a multiple correlation analysis of organ weights and other data obtained by necropsy of cottontail rabbits. Second is the determination of movements of cottontail rabbits in space and time by. tracking radio-equipped rabbits. The computer will be used to plot movements on paper, using azimuth data from the field. (GIen C. Sanderson)

1987-64049 T Electrical Engineering. Spherical and Related Antenna Arrays. The objective of this research is to investigate large antenna. arrays of spherical and related geometry useful for space communication. Use will be made of the rotational group in relating the spherical wave function to the antennas on the sphere. Randomly distributed antennas on the sphere will also be considered and the Monte Carlo Method will be used to verify the theoretical prediction.. (Y.T. LO)

1988-64050 Horticulture. Market Grades and Standards for Cut Roses. The problem is to identify factors determining quality in the important commercial varieties and types of cut roses, and to develop specifications for market grades and standards for these varieties and types of cut roses. Ten different measurements were made on eight stem lengths of Better Times rose over a period of thirteen months. The computer will be used to analyze 
the data and select those quality factors that best correlate with increase in stem length. The data will also be analyzed for the effect of seasonal variation due to environmental changes on those quality factors. (culbert)

1989-64051 State Water Survey。 Atmospheric Pressure and Wind Analysis. This program facilitates manual analysis of streamines, isotachs, and isallobaric fields. It also provides a time-dependent distribution of winds, pressure tendencies, and kinetic energies which can be analyzed statistically. The input consists of coded. synoptic messages from which wind direction, wind speed, and pressure are read. Necessary data conversions are made and the following statistics are computed and printed: (1) Averages and standard deviations of wind direction for each hour; (2) Average kinetic energy and its standard deviation for each hour; (3) Averages and standard deviations of the pressure tendency for each hour; and (4) cross-correlations between wind direction, kinetic energy and pressure tendency. The program punches cards of the converted input data for use in further statistical analyses and prints out maps of wind direction, wind speed, kinetic energy, wind components, and pressure changes. (Robert Sinclair)

1990-64052 T Chemistry and Chemical Engineering. Crystal Structure Analysis. The research problem concerns the solution of the structure of chemical compounds by $x$-ray diffraction.techniques. The electron distribution in a crystalline solid is a periodic function in three dimensions and this may be represented mathematically by Fourier series. The coefficients to be used for the terms in the series are related to the intensities of $x$-ray beams diffracted by planes of atoms in the crystalline solid. The computer will be used to sum the Fourier series, which usually involve several thousand times, over a three dimensional grid. In addition theoretical intensities for the diffracted beams will be computed and compared with the observed intensities. Least squares analysis programs will be used to obtain the best fit to the observed intensities as a function of the coordinates of the atoms in the solid. (J.A. Stanko) 
1991-64053 Theoretical and Applied Mechanics. Least Squares Reduction of Bond Study Data. The computer will be used for the reduction of data obtained in the study of the bonding of deformed wire to the concrete in which it is embedded. Library routine LSQ2 will be used to obtain a least square polynomial fit for the data. The data were obtained from a series of approximately 200 "püll-out" tests on different types of wires. (Harry Kohler)

1992-64054 T Mining, Metallurgy, and Petroleum Engineering. Plastic Impact. The propagation of torsional impact waves, both elastic and plastic in a thin cylinder of metal will be studied. The strain hardening is considered, related to the movements of dislocations. The second order partial derivatives system whose boundary conditions are given by the characteristics of the impact loading, will be integrated, with.reasonable values of the physical parameters, to give the shape of the propagating pulse. (Vassort)

1993-64055 T Agricultural Economics. Farm Planning Laguna. The problem is making optimum farm plans for specified levels of income for farmers in Laguna province. Linear programming techniques will be employed. The data will be obtained from farm records. (Pres Kuhonta)

1994-64057 T Chemistry and Chemical Engineering. Fitting of the Time Constant of a Carr-Purreil Train Influenced by Quadrupole Relaxation to a Theoretical Formula. The program to be used in this project fits the time constant of a Carr-Purcell spin echo train as a function of the spacing between the 180 degree pulses to a theoretical formula. A trial and error method is used, to fit the data. This will enable the coupling constant between a spin $1 / 2$ nucleus and a nucleus with a quadrupole moment to be calculated simultaneously with the relaxation time of the quadrupolar nucleus. The program also calculates the error in the parameters by introducing noise into the experimental data, refitting the data, repeating 75 times, and then taking the standard deviation of the parameters. It is proposed to study at least 5 chemical systems in this manner. (E. S. Gore) 
1995-64059 Chemistry and Chemical Engineering. Computer Processing of Mass Spectral Data. The IILIAC II computer and the CalComp plotter will be used to plot mass spectral data. At present this is done by hand, and is very time consuming and error prone. The program will read in the measured peak heights, normalize them so that the highest peak is $100 \%$, and then plot the spectrum. Since all spectra will be drawn with the same criteria, cross comparisons and interpretation will be made easier. (K. L. Rinehart)

1996-64060 Bureau of Educational Research. Concept Teaching Strategies. A procedure has been developed for identifying and categorizing the information content of recordings of high school class lessons. This procedure was used in the construction of five alternative programmed texts for teaching each of two social science concepts to high school students. The programmed texts were used with a representative sample of 470 high school students, and the results of a criterion test administered four weeks later were factor-analyzed to provide factor scores on student achievement. These factor scores were used in several three-way analyses of variance to ascertain main and interaction effects between students' prior level of knowledge, pre-treatment exposure to related content, and alternative programmed texts. The results will be used to test a set of hypotheses concerning students' acquisition of conceptual knowledge in the classroom. (Graham Nuthall)

1997-64061 Speech and Theatre. The Effects of Self-Contradictory Communications on Attitude Change. The purpose of the study is to investigate the effects of:a self-contradictory on attitude change. The basic design of the study is a $2 \times 2 \times 2$ analysis of variance. Independent variables are: (1) an inconsistent and consistent persuasive message; (2) the initial attitude of the subjects towards the concept supported in the message; (3) the open and closed-mindedness of the subjects. The following dependent variables are used: (1) attitude change towards the concept supported in the message; (2) attitude towards the message; (3) attitude towards the source of the message; and (4) perception or non-perception 
of inconsistency in the message. Predicted results are as follows:

(1) the inconsistent message will elicit less favorable attitude change than the consistent one; (2) there will be an interaction between consistency-inconsistency and the initial attitude of subjects; (3) there will be an interaction with the open and closed mindedness of subjects. (John Schunk)

1998-64063 Speech and Theatre. The Effect of Emotional Language on Attitudes. This experiment is designed to test the effect of emotional language on attitudes. Emotional. language is defined in terms of the evaluative and dynamism (potency and activity) dimensions as measured by Osgood's semantic differential. Four types of adjectives which are descriptive of a person's character and activities are used, for example in one speech the person is described as "outstanding" (good and dynamic), in another as "decent". (good and listless), in a third as "contemptible" (bad and dynamic) and in a fourth as "worthless" (bad and listless). This study also seeks to discover interaction effects between original attitude toward the person described in the speech (positive, negative, or neutral) and any of the four types of language. A $3 \times 2 \times 2$ analysis of variance is required. Subjects are students enrolled in Speech 101.

In addition to attitude change as a dependent variable, this study also seeks interaction effects on perception of emotional language and acceptance of the language as being fair and accurate.

It is expected that the dynamic language will be more persuasive than the listless in all three original attitude groups. It is also predicted that those with original attitudes opposite to the one presented in the speech will more readily perceive the dynamic language and will judge it to be unfair and inaccurate, but will nevertheless be somewhat persuaded by it. (Helen H. Franzwa) 
1999-64064 T Physics. Equilibrium. Equation of Cubic Crystal. The purpose of the problem is to find the approximate solution of the equilibrium. equation of a cubic crystal. This șolution is homogeneous in $\mathrm{x}, \mathrm{y}$, and $\mathrm{z}$, and plays the same role as $\frac{1}{\gamma}$ of the Laplace: equation. The computer will be used: (1) to find numerical roots of a sixth order algebraic equation whose coefficients are functions of the polar coordinates $(r, \theta, \varphi)$; (2) to fit the numerical roots as functions of the polar angles $(\theta, \varphi)$. These six roots of the algebraic equation as functions of $\theta$ and $\varphi$ give an approximate solution of the equilibrium equation. (K. H. Lie)

2000-64065 T Educational Administration. Comparative Perceptions of Educators in Illinois School Districts. The objective of this doctoral study is to compare (and test for significance) the perceptions of teachers, principals, and central office personnel regarding the importance of data that is frequently used in teacher selection (for purposes of employment). Much of the argumentation which is found in the literature (and in actual school practice) about who should be a participant in teacher sëlection -- peer, principal, and/or superintendent -- rests on the assumption that perceptions of these groups do or do not differ as to types of teacher selection data that are important to consider in the selection. procedure. This study focuses on this very question. Three hundred sixty educators in the state of Illinois were sent, by mail, the instrument called Elementary Teacher Selection Index, which was developed specifically for this study. Instruments were returned by 87.5 percent. Stratified random sampling was the procedure employed. The instrument contained twenty-four different types of teacher selection data ( $e_{\circ} g_{\circ}$, marital status, honors and. awards received, work experience, etc.), each of which:was repeated (and matched) five times within the context of the other items. Items. were set in blocks of five, and respondents were asked to rank the five items in the block (or set) by its importance in teacher selection (relative to the other four in the block) with a 1 signifying very important (or most important), 2 second in importance, and finally 5 the least important. The five separate 
weightings given an item (throughout the instrument) are summated (by computer) into a score which is then used for all computations that are necessary to determine significance of group differences in response (per item). Twentyfour one-way analyses of variance will be employed, and ninety-six two-way analyses of variance will be used. The level of significance will be set at the 5 percent point. The design will be a factoral or balanced design where each cell is equal to $N$, where $N$ is always greater than one. Independent. variables will include formal school position, age, sex; experience, and school district size, and the dependent variable will be the response on the instrument. per item. The computer will be used: (1) to provide the sum (or score) for each of the twenty-four items in the instrument; (2) to conduct tests of significance by analyses of variance; (3) to compute the intercorrelations of twenty-four variables and place them into a matrix, one such matrix for each of three groups of respondents; (4) to provide the mean response per item by several groups of respondents; and (5) to provide many of the calculations necessary for hand computation of significant "gaps" between individual means (Tukey's method). (Donald R. Draayer)

2001-64066 Horticulture. Respiration of Isolated Mitochondria. The respiratory characteristics of isolated mitochondria of the tomato plant, Lycopersicon ealentum Mill., are being determined as part of the research program on the physiology and maturation of the tomato fruit. The respiratory rates under a variety of experimental conditions are measured with an oxygen electrode and are obtained on a graph recorder. These rates, the temperature, the mitochondrial nitrogen, the level of phosphate acceptor, and the oxygen consumption of respiratory state three are read into the computer. The output includes respiratory control ratios, phosphorylative efficiency, oxidative efficiency, ratios of the rates under differing experimental conditions, and averages of all of these. An average experiment has fifteen items of data from. which an average of one hundred items are obtained. The computer results will be used to evaluate methods of isolation, the effects of respiratory inhibitors and regulators, and the variation of respiratory activity during ripening of the tomato fruit. (McCollum) 
2002-64067 Economics. Moment Simulation. Various well-known frequency distributions will be constructed for the purpose of empirically testing the usefulness of a.moment approximation for such distributions. Samples for such testing will be generated from these parent distributions, and the adequacy of the approximatox checked by other statistical techniques. (D. Aigner)

2003-64068 T Physical Education for Men. Effects of Dietary Supplements on Selected Cardiac Intervals in Matched Groups of Young Boys. Three matched groups of young boys (aged 6-13 years) were given dietary supplements during the program of the University of Illinois Sports Fitness School in the summer of 1965. The study involves the measures of selected cardiac intervals done on a 5-channel electronic apparatus in the Environmental Lab and the comparison of these measures between $\mathrm{T}^{1}$ and $\mathrm{T}^{2}$ testing. The statistics involved is a finding of the differences between the groups in the different treatments and the two different testing periods. A t-test is then used to determine if there is significance between the group differences. (Iinda Cundiff)

2004-64069 Entomology. Analysis of Sexual Behavior in Epicauta. The general problem is a. study of patterns of sexual behavior in insects of the family Meloidae (blister beetles). Specifically, the present study is an analysis of an experiment in which a series of males of two closely related species. of the genus Epicauta were given, individually, a duo of females consisting of one homospecific female and one heterospecific female. The information desired is: 1) do males of a given species show preferential behavior with homospecific females; 2) how is such preference affected by body-size relationships of the two females and the male? The data were obtained in Argentina in 1964 under rather primitive conditions. They consist of records of total courtship time with homospecific and heterospecific females, number of bouts of courtship with each, and amount of time mounted and oriented with each. The SSUPAC multiple correlation program will be used in an attempt to investigate the relative importance of the various size relationships in determining male behavior. Some specific 
questions are: .1) are the homospecific-larger females preferred by males because such females are absolutely large or because they are larger than the heterospecifics; 2) does the size of the male relative to that of the homospecific female have an effect on preference level; and 3) is there any difference between males of the two species in regard to their levels of preference for homospecifics and the effect of size on these levels? (R. B. Selander)

2005-64073 T Agricultural Economics. Leadership in the Fischer Community. This research.project is a pilot study on the identification of the leadership structure of Champaign county, in which Fischer is located. Two methods of identification of leaders are to be compared. A reputational approach, in which reputed leaders will be interviewed by means of a questionnaire, will be compared to a set of interviews obtained from a random sampling approach. The leadership structure will be ascertained by frequency counts of names from the questionnaires. . Socioeconomic characteristics, visiting patterns, reference patterns; and organizational affiliation will be compared for the two groups of people. Statistical tests of the significance of differences will be calculated. (D. R. Smucker)

2006-64074 Small Homes Council, Bureau of Residential Construction. Heating Loss Research. During the three months of December 1965, January and February of 1966, data on heating research was taken at two elementary schools in Chicago, Illinois. The data was recorded daily. by the engineer in charge and later compiled into tabular form from which graphs are to be drawn for temperature differences versus heating therms. The computer will be used to select and draw the best-fitting straight lines for the graphs. The computer will be programmed to use the method of least squares. The computer rèsults will be used to compare the heat. loss characteristics of the two schools. (Anthony Dasta) 
2007-64075 T Special Education. The Relationship of Vocational Outlook and Special Educational Programs for Adolescent Educable Mentally Handicapped. This study makes an evaluation of the thirteen high school programs for the educable mentally handicapped (two or more classes per high school) in Illinois, and investigates possible relationships of student vocational outlook and program elements. Approximately thirty-two classes and four hundred students are included in the study. Other elements - family background, intelligence, sex, race, age, etc. - will also be examined to determine possible relationships to vocational outlook. Rank correlations, median tests and chi square techniques will be obtained through use of the computer. (Philip R. Jones)

2008-64076 T Education. Correlates of Teacher Prediction. The object of this study is to explore the relationships of intelligence, creativity, and achievement to teacher prediction of expected success of sixth grade pupils. Multiple correlation is to be used in order to determine: (a) the amount of variance contributed to teacher prediction by each variable, and (b) the Beta weights of each variable and its subsections. Teacher prediction was obtained by a seven-rating-position questionnaire. Creativity scores were the arithmetic sum of 3 subsections, fluency, flexibility, and originality, of each of 7 tests in experimental form. School grades of 5 types were assigned weights and translated into rankings. Test results obtained from school records were: (a) achievement: reading comprehension, arithmetic reasoning, spelling, and median; (6) IQ and its MA equivalent for each student. There were 29 students tested in the study. Twenty teachers rated these students. (Perry)

2009-64077 Forestry. McIntire-Stennis Forestry. 55-303. Timber inventory data from several natural areas owned by the University of Illinois will be compiled by means. of frequency tables. Parameters used to quantitatively describe the areas will be generated. Tree species, condition, and size information will be used to obtain numbers of trees and basal area volume per acre. Parameter conversions will be executed by matrix algebra.

(Boggess) 
2010-64078 T Materials Research Laboratory. Numerical Solution. of the Thermal Diffusion Equation. The computer will be used to obtain a numerical solution to the thermal diffusion equation in one dimension. The equation is a general parabolic partial differential equation, and arises when a material is placed in a temperature gradient. The method of finite differences will be used to solve the equation. The computer results will be compared with the experimental results in order to determine important physical quantities. (W. Mock)

2011-65001 T Electrical. Engineering. Midas Modification. Midas is a FORTRAN. II program which simulates analog computer solutions for differential equations. It will be modified to allow iterative solutions for problems with unknown initial conditions. After the program has been debugged, a few runs will be made using invented data for easy checking. This will involve choosing a simple differential equation with known results and letting the program find an initial condition. When this checks out satisfactorily;; a run will be made to compare a Midas solution to experimental results. (William Stoltey)

2012-65002 Vocational and Technical Education. Problems Unique to Implementing Home Economics Occupational Education Courses. This project is an independent study for course number 449 in Vocational and Technical Education. Home economics now, has the responsibility to develop courses and programs designed to prepare qualified interested persons for gainful employment. This project is designed. to identify the problems unique to implementing home economics occupational education courses. Once specific problems have been identified materials may be developed which will prove helpful to gainful employment course planners and coordinators. Programs are being planned or are already underway in secondary schools in 35 of the 50 states, according to information obtained from the 50. state supervisors. Data for the project have been obtained from the persons involved with these programs. The computer will be used to determine raw scores and percentages of each of the responses. (Margaret A. Dewar) 
2013-65003 T.. Political Science. Political Survey of Indonesian Students. A series of interviews. were conducted with Indonesian youth during the early months of 1965 in order to obtain. supplementary data for a study of the political. socialization process in Indonesia. Information derived from 116 of these interviews was coded on 43 variables. The distribution of subject responses on each of these 43 variables and cross-tabulations for every pair are to be determined, with relationships between pairs measured in terms of the product-moment correlation coefficient. Results will be useful in checking the validity of certain hypotheses. about the development of political orientations among Indonesian youth as well. as in the generation of possible new hypotheses on this subject. (S. A. Douglas)

2014-65004 T Department of Computer Science. Analysis of Autonomous Arithmetic Structures. Figures of merit of autonomous arithmetic structures and their subsystems will be investigated to determine the most effective structure and to quantitatively relate structural complexity to computational speed. Simulation and probalistic Markov Chain analysis will be the major methods of analysis. (M. J. Pisterzi)

2015-65005 Speech and Theatre. Self-Esteem and the Ego-Ideal. This project is an attempt to quantitatively measure the difference between the subjects' perceived self-esteem and his ego-ideal through the use of Semantic Differential technique. The subjects describe themselves and their egoideals on twenty scales. The scores are factor-analyzed by the Centroid Method, and the factor loadings serve as an index of the amount of variance contributed by each item. This is part of a larger project measuring the relation of self-esteem to persuasibility. (W. T. Page)

2016-65006 T Psychology. Interpoint Distances Be:tween Stimuli. The data for this problem were obtained in a psiychology experiment. These data are assumed to represent some monotonic function of the interpoint distances between points representing stimuli。 A previously. written program - MDSCAI - will be used as a nonmetric method for multidimensional scaling in analyzing the data. 
The data are read into the computer as. $27 \times 27$ matrix, where each cell is the number of confusions occurring for a specific pair of the 27 stimuli. The technique is an iterative one in. which the $\underline{n}$ objects are converted from an n-l dimensional simplex into a solution in a minimum number of dimensions. This solution provides a set of interpoint distances, their dimensionality, coordinates for the points, and goodness of fit for the solution. (Joseph Lappin)

2017-65007 T Bureau of Economic and Business Research. Economic Evaluation of Pricing Water Supply in Illinois. The study is to review and evaluate the current practices in pricing municipal water supply in Illinois. It considers the practices followed by public and private agencies in Illinois in pricing water for various uses to determine how such practices affect the selection and scheduling of water improvement projects as well as the current use of Illinois water. A two stage mailed questionnaire is administered to collect the required data. The computer will be used in the survey statistical analysis. Cross tabulation, variance analysis, measures of associations will be applied to the questionnaires data along with correlation analysis. (Hamiy H. H. Afifi)

2018-65008 T Physics. Quark Scattering. Quark-antiquark scattering will be investigated from the bootstrap point of view by means of (a) the so-called. "N over $D$ " integral equations, and (b) generalized potentials. Exchange of "scalar", pseudoscalar; : and vector mesons will be assumed in both possible representations: of a group known in group theory as. "SU " (Special unitary group of rank three). The same particles will then be searched for in the direct channel. Method (a) requires use of the computer primarily for the numerical evaluation of integrals. Method (b) involves a recursive search for the eigenvalues of the Schrödinger differential equation. (Laẉrence King) 
2019-65009 T Education. The Influence of Interpretative Ideas on Mental Processes of Nine and Eleven Year Old Children in Science. The study deals with the relationship between interpretative ideas developed by the teacher and mental processes. of nine and eleven year old children working with a given sequence of science activities. Two treatment levels, designated "pupil guided" and "teacher guided" were used to differentiate the experimental groups. The experimental groups.worked initially with such materials as wooden blocks, paper and clay, prior to the laboratory sequence. Other groups differing in the amount of experience were used as controls.. One designated as the laboratory control group worked only with the laboratory materials while the other 'group worked exclusively with appraisal materials.' Response frequencies, corresponding percentages, with mean scores for both positive and negative factors related to concept formation will be determined. The frequency of response means for the respective groups will be subjected to an analysis of variance. Tallies related to behavioral units will be subjected to the chi square and t. tests of significance. In summary the design for this investigation is of the dichotonomous type divided into high and low treatments of two maturity levels. The statistical analyses mentioned above will be used in making comparisons among the experimental and control groups. (R..B. Burns)

2020-65010 Forestry. Hatch Forestry -- Preservation. Wood Products. This is a $2 \times 2 \times 5$ factorial problem involving. the interacting effects of solution temperature; wood moisture content, and treating pressure on the moduli of rupture and elasticity of Douglas fir. The data will be treated by an analysis of variance. The data. were obtained in laboratory experiments conducted on the campus. The results will be helpful in designing methods for applying preservative and fire-retardant chemicals to wood. (C. S. Walters)

2021-65011. Sociology• Religious Living Study. The research problem is to analyze the data collected from approximately three thousand respondents interviewed on religious attitudes and behavior. The data were collected from a probability sample of Illinois adults. The computer 
will be used to establish various relationships between religious practices and beliefs with other survey variables such as family planning, community participation, and various demographic variables such as age, sex, race, income, education, and so on. For the predicted relationships, the analysis will consist of cross tabulations and the comparisons of means, standard deviations, and proportions. It is also likely that measures of association (lambda and gamma) will also be computed. For the analysis of predicted relationships, the SSUPAC library of programs will be used. For exploratory analysis of the data in search of interunion effects, the AID \#2 program will be used. This program is a University of Michigan computer program that uses a ratio of between sum of squares to total sum of squares to locate interaction effects in survey research data. (The University of Illinois Statistical Service Unit is now in the process of converting this program for use in the SSUPAC library of programs.) (Bernard Lazerwitz)

2022-65012 T Theoretical and Applied Mechanics. Cylinder Subject to Bending and Pressure. An initially straight cylindrical shell subject to bending, may collapse by local ovaling of the cross-section. Cylinders constructed of a low modulus material and having a relatively large thickness to radius.ratio have this property. It is desired to determine the critical bending moment for such failure for various geometric properties of cylinders, and the effect of lateral pressure. The non-linear equations derived for thin shells are employed in the analyses. Their complexity suggests an inverse solution, and assumptions on the radial, tangential and axial displacements of the shell are made. On substitution of these displacements, one obtains equations in terms of the displacement parameters. The matrix of the coefficients of the parameters is minimized by computer for various values of the bending moment parameter, geometry parameters, and the pressure parameter. (Peter Janzen)

2023-65013 Mechanical Engineering. Hydrodynamic Gear Load Capacity. In this investigation the pressure distribution in oil-films separating gear teeth are computed. With these pressures the hydrodynamic load capacity is calculated. The effect of various profile approximations are being compared 
as. well as the effect of the surface curvature terms in the basic Reynolds . equation. Pressures are obtained using trapezoidal integration to integrate pressure gradients. The constant of integration in this process has to be evaluated using the Reynolds boundary condition. Interval halving iteration methods are used to establish the correct film thickness to give the proper load characteristic. (R. W. Adkins)

2024-65014 T Physical Education for Men and Graduate PE. The Effect of Two Different Exercise Programs on Various Cardiä Intervals in Middle Aged Men. The effect of an endurance running program and a weight training program on various cardiac intervals of middle aged men was the main problem under investigation in this study. $:$ Four different heart waves were simultaneously recorded on each subject. From these tracings the cardiac cycles were measured with vernier calibers. These heart waves were recorded at rest, and at 2 1/2 min., 5 min., and 10 min. after a two min. steptest. The training programs lasted for twelve weeks. There were 8 subjects in each training group between the ages of 25-50 years. Analysis of co-variance will be used to determine the effect of the $2 \mathrm{~min}$. step test on the intervals before and after training. T-tests for the difference between means will be used to determine the effect of the $2 \mathrm{~min}$. step test or the amount of change in the intervals before and after training. Pearson correlation will be used to determine the reliability of the test. (Stephanus du Toit)

2025-65015 T Physical Education for Men and Graduate PE. Effects of Distance Running on Endurance of Boys. The purpose of the research problem is to dețermine progressive changes in endurance performance and metabolic potential of junior high school boys exposed to a distance running program. Seven experimental. and eight control subjects have been selected from Thomas Jefferson Junior High School, Champaign. Experimental subjects participate in fifteen weeks of training taken three days per week, thirty minutes each training: session. Selected physical performance tests are administered before and after the experimental program, and serial determinations are made on endurance running ability and energy metabolism 
variables every five weeks. The use of the computer is essential for obtaining the following information: (1) Descriptive statistics of groups, i.e., means, variances and standard deviations. (2) Sampling techniques: whether the variables are normaliy distributed and whether the sample is representative of the population from which it. was drawn. (3) Correlation and regression analysis: (a) intercorrelation matrix and bivariate regression analysis; (b) multiple correlation and multiple regression analysis; (c) causal analysis. (4) Significance testing: (a) to study the relationship of the sample to population parameters; (b) to determine differences between and within groups in terms of means, variances, and correlation data before, during, and after training. (5) Reliability tests (Pearson Product-Moment Correlation). (6) Development of standard. score tables for junior high school boys. The results of the computer analysis will assist in testing the established hypotheses pertaining to the effects of longitudinal physical training on the health and growth patterns of early adolescent males. (Michael A. Sherman)

2026-65016 Material Research Laboratory Calibration of Fused Quartz Pressure Gauge. The calibration data between angular output and pressure for a fused quartz pressure gauge is to be extended using polynomial interpolation. The calibration data will also be used with an eight term series expansion in a log (pressure) formula for the helium-4 vapor pressure scales to form a table converting angular output to temperature. This is needed to make efficient use of the fused quartz pressure gauge related to datataking for research in super conductivity. This is the primary method of measuring temperature for all research in the high field super conductivity group. (Ival Toepke)

2027-65017 T Theoretical and Applied Mechanics. Lateral Buckling of an Intermittently Stiffened I-Beam. The Rayleigh-Ritz method is used to solve this problem. The energy expression is. written for the intermittently stiffened I beam in a general deflected state. This deflected state is given the shape of some assumed trigonometric series. whose unknown coefficients are generalized coordinates. According to the principle of minimum potential energy the 
second variation of the energy must equal zero at the buckling load. For this to hold, the determinant of the coefficients of the generalized coordinates must equal zero. The computer will be used to find the value of the eigenfunction which makes the determinant zero. (W. Wayne Siesennop)

2028-65018 T Physical. Education for Men and Graduate PE. The Effects of Training on the Hemodynamics of the Circulatory System, Oxygen Consumption, and Eosinopenia in Middle Aged Men. The problem is a. study of the effects of physical training on the hemodynamics of the circulatory system, oxygen. consumption, and eosinopenia in middle aged men. There are three experimental groups and one control group with varying numbers in each group ranging from seven to ten subjects. There will be approximately forty variables with $\mathrm{T}^{\mathrm{l}}$ and $\mathrm{T}^{2}$ on each variable. The groups are unmatched; therefore, an analysis of covariance will be used to acciount for the differences in groups on $\mathrm{T}^{\mathrm{I}}$. In addition, SSUPAC programs will be used for the determination of the reliability of measures and intercorrelation of variables. (David E. Cundiff).

2029-650i9 Psychology. Analysis of Color Pigments. A prior investigation has shown that the spectrophotometric reflectance curves (from 400 to $700 \mathrm{mu}$ ). of pigments can almost always be expressed as a linear weighted function of three fundamental curves; stated differently, a pigment's spectrophotometric curve can almost always be drawn if only three points are known. The present investigation seeks to apply this finding to two areas: (1) If it is assumed that the retinal light-sensitive pigments are also reducible to three fundamental curves, then, irrespective of the nature of the three retinal pigments, they must be linear transformations of the same fundamental curves. An eigenvector analysis of the known retinal pigment curves ought to lead to "fundamental sensation curves" indicating the color mixing properties of the visual apparatus. (2) Printing inks may be reduced to the weighted sum of three fundamental independent curves, as other pigments. The specification of printing. inks in terms of independent parameters ought to enable a prediction of the requisite dot-areas (densities) in the final printing plates. Data from a wide range of printed colors have been obtained by densitometers and spectrophotometers and prediction will be attempted by a least squares solution of many simultaneous equations. ( $\mathrm{J}$. Cohen) 
2030-65020 T Institute for Research on Exceptional Children. Parental: Assistance and Class Room Achievement, of the Retarded Child. This research aims to detect relationships between parental ways of dealing with an educable retarded child at. home and the child's achievement in a. special education class. The research sample consists of 36 families, divided into four comparison groups, each with nine families. The comparisons. were based on higher versus lower gain scores in school achievement and high versus low socioeconomic classification scores. Tape recorded sessions of interviews with each parent and of maternal teaching sessions provided the raw material for the investigation which was then scrutinized by means of a content analysis. Nine main categories were considered in the content. analysis: (1) persons mentioned; (2) dealings with the child; (3) subject matter of the child's activities; (4) regularity and frequency of the activities; (5) indicated problems; (6) general references to the school situation of the child; (7) comparisons with a selected non-retarded sibling; (8) parental ways of actually teaching the retarded child at home. The data were recorded within sequences of numerical codes, each code representing a single count. of an identified meaning unit. In general one code per word of the transcript. was used. The computer will be used to process this data. The first approach would be typified in case studies, summing up the total frequencies of each code singly mentioned per father and per mother for each of the subjects (resulting in 72 case summaries). The second approach would combine the total scores per case in an Analysis of Co-variance with the gain scores and with socio-economic scores. (Dorothea Rau)

2031-65021 T Material Research Laboratory. Thermodynamic Properties of Crystals. This replaces problem specification number 47048. (Ralph Simmons)

2032-65022 - T Physical Education for Men and Graduate PE. Cardiac Performance Measures. This problem is an investigation of selected cardiovascular measures and their performance under various conditions of stress. For seventy-five subjects, there are about twenty measures of eosinopenia, oxygen intake, and cardiac cycles, at three different time periods in 
relation to exercise and recovery. The analysis required includes: (1) multiple and partial correlation and regression; (2) additional indexes on contrived" variables after the inspection of the first parts of the analysis; and (3) distribution analysis. (E. Sloniger)

2033-65023 T Chemistry and Chemical Engineering. The.Effect of Vibrational Energy in a Chemical Reaction. In order to calculate the effect of vibrational energy in a chemical reaction the potential surface in the reaction region is approximated by a sector of circle curvillinear channel. An elastic collision is assumed. With the incident point $\rho_{i}$ and the incident angle $\varnothing$ given, the reflection point $\rho_{f}$ and reflection angle $\delta$ in the output channel are easily calculated. Then the change of vibrational energy of the incident particle in passing through the curvillinear channel, $\Delta \mathrm{E}_{\mathrm{vib}}$, is equal to

$$
\frac{\Delta \mathrm{E}_{\mathrm{vib}}}{\mathrm{E}_{\text {total }}}=\cos ^{2} \delta-\cos ^{2} \phi
$$

Since the change of vibrational energy is related to the reaction rate, the effect of vibrational energy in chemical reaction can be determined. (Shiou-Fu Wu)

2034-65024. Bureau of Educational Research. Study of Student Lifeways in a Puerto Rican High School. As part of a study of student lifeways in a secondary school in Puerto Rico, a questionnaire in Spanish was developed and administered to all the students in the high school. A. second questionnaire was developed and administered to the teachers. These questionnaires were used in conjunction with four months of participant observation in the high school. The questionnaires include selected questions from two different: studies of North American high schools which were translated into colloquial Spanish, as well as questions designed to test hypotheses developed during the course of participant observation. The computer will be used to compile information on the collected data. (J. H. Burnett) 
2035-65025 T. Civil Engineering. A Study of Air-Blast-Induced Ground Motions. Wave propagation calculations will be accomplished for correlation with free field ground motion data from a large scale high explosive test. The static Boussinesq solution will be obtained for the specific loading conditions of the test to obtain an estimate of the spatial attenuation. One-dimensional wave propagation in a non-linear non-elastic material. will be studied for an arbitrary step-wise varying. loading function. (Harry E. Auld)

2036-65026 T Electrical Engineering. The Sensitivity of Cascaded Bridge T Networks. An important consideration in the synthesis of electrical networks is the effect of small changes in the network elements. The objective of this thesis is to study how these changes affect the network functions for an important class of networks, the constant resistance cascaded bridge $T$ networks. In this problem, the sensitivity of voltage transfer and driving point impedance functions with respect to various parameters in a bridge $T$ cascaded network will be studied. A low-pass filter will be designed using cascaded bridge $T$ networks. The magnitude and phase characters of the filter will be investigated for a region of low frequencies. (Mehmet Ermis)

2037-65027 T Physiology. Evaluation of Ionic Fluxes. This research concerns the movements of various ions across biological membranes. Both labeled and unlabeled ions will be used. Data will be fitted to various models using (1) least squares analysis and.(2) simultaneous differential equations. In adiition, simple operations such as correcting radioactivity counts for background and decay will be performed. (Wm. R. Lieb)

2038-65028 State Water Survey. Surface Data Mapping. For a study associated with radioactive fallout studies, computations and mapping of several meteorological parameters are done for a network of U.S. Weather Bureau observation stations. Input data are received in synoptic code form and the computer uses appropriate formulas to expedite the computation of ( 1 ) humidity mixing ratios, (2) potential temperatures, (3) pressure and (4) temperature at the lifting condensation level, (5) equivalent potential temperatures, (6) wet bulb potential temperatures, (7) specific energies, and (8) total energies. : The printed output consists of plotted maps for each of the eight parameters. These will be used to follow air masses along their three dimensional trajectories. (Pieter Feteris) 
2039-65029 T Civil. Engineering. Large Deflections of Thin Plates. This research problem.investigates the effects of changes in geometry resulting from large plastic deformations of thin plates. The continuous plate is represented by a discrete model which is analyzed by consideration of the large-deformation theory of strains. The analysis of the discrete model takes the form of simultaneous non-linear equations of equilibrium in terms of displacements. These equations are solved recursively by using the solution to the analogous linear equations of equilibrium for initial values of displacements。 (James G. Crose)

2040-65030 Industrial Administration. Department Store Customer Research. The research problem involves an analysis of the household characteristics, shopping behavior, and purchase patterns of the charge and cash customers of a specific department store. The specific customers used in this study are those who made one or more purchases in this store during a sampling time period. Analyses are based on the segmentation of these customers into two major segments--cash customers and charge customers--as determined by their purchases. These two major segments will be refined into numerous sub-segments in the process of their identification and the analysis of household survey data. From analysis of the similarities and differences of the two customer segments, management should have the basis for evaluating. whether unique appeals appear desirable for a particular segment, and if so, which ones. In effect, the question to be tested is whether market segmentation by the kind of transactional relationship is a significant one for the management. of. a department store. SSUPAC programs will, be used for this analysis. (Herbert. I. Ross)

2041-65031 T Mining, Metallurgy, and Petroleum Engineering. Solution of Higher Order Partial Differential Equations. It is desired to solve various elasticity problems of plane stress. and use the models solved to explain failure and faulting patterns in geological structures. The problems will be mainly those of bodies having notches or protrusions. The biharmonic equation $\nabla^{4} \phi=0$ will be solved by using standard relaxation methods. In a few cases, the function $\varnothing$ will be known and it will be necessary only to plot it. (John R. Sturgul) 
2042-65032 T Nuclear Engineering. Gamma-Electric Cell Research. This problem. involves the calculation of the output currents and efficiency of the gamma-electric cell $(\gamma-E C)$. The gamma-electric is a solid state device . which directly converts gamma ray energy into electrical energy by using the Compton effect. Theoretical calculations will be compared with experimental data obtained at the University. of Illinois Triga Reactor. The basic program will involve the solution of integral equations. The integral will be evaluated using: Simpson's rule. The output will give $\gamma-$ EC current as a function of voltage, cell geometry, and materials of construction. (H. T. Sampson)

2043-65033 T Physical Education for. Women. Glover Fitness Test. Norms. Twenty-three hundred children, ages six to nine, from twenty-one Illinois schools were tested on the Glover four-item physical fitness test battery. This: battery consists of a standing broad jump, seal crawl race for twenty: feet, modified. sit-ups for thirty seconds, and a four hundred foot shuttle race. Centile norms for each test item were developed for each age group; according to sex. In addition, the reliability coefficients for each item were determined by the Pearson product-moment correlation method, and analysis of variance was used to determine whether or not significant differences existed in each of the following sets of conditions:

(1) children taught by a classroom teacher and those taught by a specialist;

(2) children who have regularly scheduled physical education and those who do not; (3) children from high, medium, and low socio-economic level families; (4) children tested in the morning and those tested in the afternoon; (5) children of various ages; and (6) boys and girls. at each age level. (Sharon Blackadder)

2044-65034 Institute of Labor and Industrial.Relations. Indian Middle Classes. The data being processed in this problem concern the origins, development, and social composition of the middle classes of India. The data. were gathered in seven separate sociopolitical surveys in India. The computer will be used initially to determine simple frequency distributions and percentage distributions of the various variables. On completion of the latter, selected variables will be subjected to cross classification and statistical tests of significance. (Joseph R. Gusfield) 
2045-65035 T Civil Engineering. Hydrograph of Moving Storm. The flow equations pertaining to the study of surface flow are derived from the continuity and momentum principles. These derived equations with a numerical procedure, boundary and initial conditions, and necessary parameters make up a mathematical model of the physical flow situation which is intended to be solved on the 7094 computer. (Marcus Nachman)

2046-65036 Physical Education for Women. Relationship of Dominance to Mental and Motor Ability. The purpose of this study is to determine the relationship of hand, foot, and eye dominance to the motor ability scores, grade point averages, and college entrance ACT scores of women physical education major students at the University of Illinois: Freshmen, sophomores, and juniors were involved in the study. Evidences of dominance were obtained for the hands, feet and eyes by the following tests: (I) Hands: (A)Writing, (B) Threading a needle, (C) Grip strength as measured by hand dynamometers, (D) Throwing a softball; (2) Feet: (A) Kicking a stationary soccer ball, (B) Taking a starting position for a dash, (C) Sitting, standing, and running to a line, (D) Recovery from a push, (E) Picking up a pencil. with the toes; (3) Eyes: (Á) Sighting through a hole in a cardboard, (B) Leading eye while writing (closest to paper), (C) Lead eye judged subjectively by comfort. A questionnaire was administered to determine whether the students have or have ever had reading, spelling, stuttering, or probation problems; or injury or defect of arm, foot, or eye. The twelve measures of dominance can best be analyzed by factor analysis to determine the important factors. Intercorrelation and point-biserial correlation will also be imployed to determine the relationship among all of the measures. (Charlotte Lambert)

2047-65037 Mechanical Engineering. Diesel. Engine Combustion Investigation. Fundamental investigation is being conducted into combustion in a supercharged diesel engine. The problem will be to make a theoretical analysis of the process and compare the actual process with the theoretical. Effects of swirl, squish, air velocity, and nozzle configuration will be analyzed. (W. L. Hull) 
2048-65038 Psychology. Propositional. Control. This project analyzes interrelationships among experimental conditions and human subjects' reports of their hypotheses and intentions during learning. The data are gathered within the Physical Environment Unit; the PLATO Laboratory of Instruction, and Kankakee State Mental Hospital. Use of the computer permits analysis of variance in numerous report measures with experimental conditions as independent variables. Correlation matrices. within and over experimental conditions are also obtained. (D. E. Dulany)

2049-65039 T Department of Computer Science. Simulation of a Content Addressable Memory. Many information processing problems involve the manipulation of large amounts of data in symbolic form. Existing methods of handling this type of problem are relatively slow and inefficient. It is proposed to design a very fast processor based on a content addressable memory, which will perform string manipulations efficiently. The computer will be used to simulate partially the operation of the content addressable memory. It is intended to emplôy the SN $\phi \mathrm{B} \phi \mathrm{L}$ language to examine suitable programming techniques. The use of the content addressable memory processor for the machine translation of languages will also be investigated. (John Hayes)

2050-65040 Vocational and Technical Education. Development of World of Work Instructional Programs. The major specific objectives of this project are: (1) to identify the specific items of knowledge possessed by youth about the occupations chosen at the time of making occupational choices; (?) to determine the relationship between changing.knowledge about occupations and changes in occupational choice; (3) to determine whether different kinds of occupation instructional programs should be designed for various student groups identified; (4) to develop basic guidelines for planning programs of instruction about the worid of work; (5) to plan and try out in a pilot center an instructional program about the world of work based on the guidelines developed. Analysis of data will include contingency tables, chi-square, and analysis of variance. (J.R. Warmbrod) 
2051-65041 Psychology• Temporal Integration. The present research concerns the problem of temporal integration. Temporal integration refers to the process whereby contingent rewards and punishments, which occur over a period of time following a response, are combined into a net value. In some way an organism must tally the short and long term consequences of the alternative responses available to him in order to decide on the best course of action. The present research is based on the assumption that temporal gradients of reward and punishment: which are established in a learning situation may be used to describe the subjective utility of an immediate or delayed reinforcement, wịth reinforcement being used in the general sense to refer to either reward or a punishment. Those factor's. which determine the height or the slope of the temporal gradient of reinforcement should also influence in a similar fashion the utility of the outcomes for the subject. Thus by specifying the factors which control the height or slope of the temporal gradient. of reinforcement for each consequence, we are, in addition, specifying the relative contribution of that reward or punishment to the overall or net utility, and providing: a general theory of conflict resolution. The computer will be used for statistical analysis of the results. The data are being obtained in either learning. or preference experiments, using rats as subjects. The computer will provide the summary information for various multi-level analysis of variance and trend analysis over trials. (K. E. Renner)

2052-65042 T Department of Computer Science. Simulation of a Multiprogrammed Input-Output System. The 7094. will be used to simulate the input-output system of a large multiprogrammed computer. Programs describing the various systems to be simulated will be written in the FORTRAN language; Monte Carlo methods will then be used to find the statistical behavior of the system, both under random conditions and under worst case conditions. One of the input-output. systems to be simulated is that proposed for the IIIIAC III Pattern Recognition. Computer. (Gerald Cederquist) 
2053-66001 T. Civil. Engineering. Behavior of Composite Beams with Flexible Shear Connection. A theoretical analysis has been developed to study the behavior of composite beams with flexible shear connectors. Since the mathematical: expressions describing the behavior of the beam are non-linear and very involved, closed form solutions cannot be obtained without the use of numerical techniques. A computer program is being developed from the theoretical analysis to study the behavior under all ranges of loading. The program will generate a. system of non-linear simultaneous difference equations, the solution of which will yield the behavior of the beam under a particular loading condition. Subsequently the program will analyse the beam under different loading conditions. This program will be applied to study the cases for which substantial experimental data are available. If the analytical solutions agree well with the test results, the method of analysis can then be used to analyse a number of beams for selected ranges of variables. The results of such an analysis may lead to a better understanding of the factors which affect the behavior of such a beam, and at the same time, provide a. sound basis on which the results of a few tests might be extended to the wider range of variables encountered in highway bridges. (T. R. Thiruvengadam)

2054-66002: T Civil Engineering. A Stochastic Model for the Rainfall-Runoff Process. The central problem is to develop an appropriate model for the transformation of rainfall to runoff. A historical record of total rainfall and runoff will constitute the basic data for the problem. The time series of these two hydrologic events will be considered as two stochastic processes. Statistical analysis of the two time series will be made to determine covariance, spectrum, and other statistical parameters of the series. These statistical characteristics will help determine the probability laws and nature of the stochastic model which appropriately represents the transformation of the stochastic process represented by the rainfall time series to the one represented by the runoff time series. Several modifications may have to be made before a satisfactory model is arrived at. This model may be used to generate synthetic runoff data from a sequentially generated rainfall data. From such synthetic records, much useful information may be obtained for the planning and design of water resources of a basin. (T. Prasad) 
2055-66003 Division of General Studies I A S. The Effect of Grades on Student Attitudes. This is a study of the effect of grades as differential reinforcement on attitudes toward the instructor, course, and self, and on speech content and delivery, in DGS 111-112. A secondary purpose of the study is to develop a reliable and valid measuring instrument to measure the dimensions of instructor and self credibility. Statistical analyses will involve factor analysis, product-moment correlation, analysis of variance, and $t$ tests. The data were gathered by means of semantic differential scales developed to assess student attitudes toward various course-related factors. The results of the study will be presented in a paper to the Speech Association of America convention this year, and will also be published in a speech journal. (Richard J. Dieker)

2056-66004 T Material Research Laboratory. Theory of Proximity Effect in Superconductors. The transition temperature of a superconductor is affected by the proximity of another (normal or superconducting) metal. The transition temperature of the combined layer is to be determined by solving the following equation:

$$
\Omega(T) \equiv \int_{-\infty}^{\infty} \chi(x) \cdot[\Delta(x)-V(x) \quad \chi(x)] d x=0
$$

where $\chi(x), \Delta(x)$, and $V(x)$ are known functions of the temperature it, and $x$ is the normal distance measured from the interface of the layer. For all experimental data available now, the superconductor used is lead, with transition temperature $7.22^{\circ} \mathrm{K}$. Since $\mathrm{T}$ for $\Omega(\mathrm{T})=0$ is therefore known to be $\theta \leq T \leq 7.22^{\circ} \mathrm{K}$, a two stage plot process is used to determine $\mathrm{T}$. Previous investigation has shown that this is a more economical method than the false position method. In the first. stage a rough plot of $\Omega(T)$ is obtained for interval of $\mathrm{T}=0.25^{\circ} \mathrm{K}$. The appropriate range over which $\Omega(T)$ vanishes is used as input data in the second stage plot, with interval of $\mathrm{T}=0.01^{\circ} \mathrm{K}$. Further refinement is unwarranted because of the inaccuracy in the experimental determinations as. well as the approximations used in the theory from which the above equation is derived. (Robert Yeh) 
2057-66005 T Nuclear Engineering. Space Time Dependent Kinetics in Multiplying Media. It is desired to calculate the space time behavior of the neutron flux in a reactor during a transient. The program is restricted to one dimensional slab geometry. Arbitrary time dependence may be introduced into the parameters in the diffusion equations to introduce perturbations or feedback into the system. The basic equations can be interpreted as the conventional two-group time dependent diffusion equations, or they can be used to describe the one-group behavior of two reactors coupled through their fluxes. (H. Hassan).

2058-66006 T Electrical Engineering. Log Periodic Filters. The computer will be used in a study of n-port log periodic filters. The computer data will be used as a standard to determine the efficiency of various methods of construction of the log-periodic filter. The main part of the program will consist in solving the hyperbolic equations for standing waves on a transmission line. (John Mickevifius).

2059-66007 Mechanical Engineering. Radiant Heat Transfer in Space. Radiant heat transfer for engineering materials in a space environment is under investigation with an ultimate goal of improving present methods for prediction of radiant heat transfer rates and equilibrium temperatures. The analysis of the system under study yields a system of integral equations which are not amenable to analytical solution techniques. The computer will be utilized to determine numerical solutions of the governing integral equations by standard iterative techniques. (R. G. Hering)

2060-66008 T Civil Engineering. Study of a Water Resource System. The problem is a study of a water resource system that is to satisfy the requirements for water supply, flood control, recreation, and low flow augmentation. A mathematical formulation is to be developed to study the behavior of the system under various operating rules and various hydrologic assumptions to determine optimum operating rules and investigate procedures of optimum design of a water resource system. (D. D. Meredith) 
2061-66009 T Electrical Engineering. Analysis of Tuned Amplifiers. This research investigates some of the major problems encountered in the actual design of a transistorized superheterodyne broadcast-band receiver. However, the goal is not to serve as a design procedure. Specifically, the loading effects of radio frequency and detector stages on double-tuned coupling circuitry are analyzed. Also, stability, image rejection, selectivity, insertion loss, and impedence reflections are optimized. Conventional mathematical methods of amplifier design are used. : Experimental data are used and compared with theoretical predictions. (John P. Salcius)

2062-66010 General Engineering. Local Deformations of a..Finite Protrusion. The project involves the solution of the first fundamental boundary value problem in a two-dimensional elastic body. The configuration consists of an unsymmetrically loaded finite protrusion from a half-plane. The methods of N.I. Muskhilishvili are then used to solve the resulting integral equations. (R. J. Placek)

2063-66011 Material Research Laboratory• Dilute Helium-Three Solutions. It is proposed to calculate certain very-low-temperature properties. of dilute solutions of $\mathrm{He}^{3}$ in $\mathrm{He}^{4}$. The calculation will start with a given effective potential between the $\mathrm{He}^{3}$ atoms which has been modified by the effects of the background of $\mathrm{He}^{4}$ atoms, and will proceed using fairly standard techniques for low density many-body. systems. Numerically, it will involve calculating a'set. of phase shifts appropriate to the aforementioned potential by either numerical integration of a second order differential equation or matrix inversion solution of linear equations - whichever proves better. Having the phase shifts, a number of one-dimensional numerical integrations will finish the calculation. Input data will consist of a few dimensionless parameters necessary to scale the equations, the potential, and the desired accuracy. (I. J. Campbell) 
2064-66012 T Physics. . $\mathrm{SU}^{3}$ Calculations of Elementary Particles. The computer. will be used to solve coupled integral equations with singular kernels. Several different techniques will be used. First the computer will be used to solve equations by an iteration procedure; the second method will utilize matrix inversion techniques; and the third will be a mixture of these two methods. The interation potential is taken by calculating the Feynman graph and Fourier transforms. The small energy or static model is assumed at first. : Later a general non-local potential is taken to solve the problem. This is a more realistic model to predict resonances. The results obtained will be used to obtain physically observable resonances from the experimental particle masses and coupling constants. The obtained results will be used to explain physical resonance states, and to establish.selfconsistency conditions on the properties of the interacting particles. (John Boguta)

2065-66013 Health Service. AMA Smoking. Freshman. Data consisting of fifteen variables concerning the smoking habits of three thousand freshmen in Rhetoric IOI at the University of Illinois are to be analyzed. The variables include Rhetoric 101 grades, cumulative grade point average, and attitudes of relatives and friends. These fifteen variables have been selected as a subset: of an original group of one hundred and two variables after examination in a previous study. T-tests will be used to compare these fifteen variables between smokers and non-smokers. (Dorothy Dunn)

2066-66015 Civil Engineering. Analytical Block Adjustment. The problem involves the simultaneous adjustment of blocks of thirty-two photographs by the method of least squares. To facilitate solution of the problem, the linearized condition equations are transformed into normal equations whose coefficient matrix far outstrips the capacity of the core storage of the machine. This coefficient matrix is then systematically compressed to facilitate solution. (H. M. Karara) 
2067-66016 T Nuclear Engineering. Repetitive. Pulsing. This research problem involves the study of the power and temperature histories of the Illinois TRIGA Reactor while pulsing at short time intervals. Theoretical models will be developed that represent. these histories and that are in the form of differential integral equations. The computer will be used to solve these equations and compare the results to experimental data. A percent error between the model calculations and the experimental values will then be found. (H. A. Kurstedt)

2068-66019 T Education. Analysis of Data from Student Faculty Relations Index. A sample of students have responded to an experimental instrument designed to measure the students' perception of faculty-student relationships. The responses will be factor analyzed both by the scales:built into the instrument and by the individual items. The analysis will be conducted both by individual respondents and by separate school means. on each item and scale. The results will be used to evaluate the instrument as a means to distinguish the differences which may exist between the perceived relationships on various campuses. (William Wellner)

2069-66020 Physics. Accelerator Design. In connection with the proposed design. of an accelerator employing superconducting cavities, it is necessary to make a number of numerical calculations of electron trajectories under a variety of assumed conditions. Since the particles will travel substantial distances in uniform magnetic fields, a modest number of iterations (of the order of 100). will usually. suffice for calculation of a. single trajectory. (C. S. Robinson)

2070-66021 Chemistry and Chemical Engineering. Crystal. Structure Studies. The project is concerned. with the determination of crystal structures by $x$-ray diffraction methods. Electron-density distributions are evaluated by the summation of triple Fourier series. Atomic coordinates are adjusted by least-squares calculations. The results. will. lead to knowledge of new types of molecular structure, of use both to the experimental chemist and to the theoretician.. (G. A. Sim) 
2071-66022 T Educational Administration. The Interaction of School and Family Factors on Selected 6th Grade Caucasian Rural. Migrants to a Large Urban School. Since World War II there has been a great migration of rural Caucasian families from Appalachia and certain southeastern sections of the United States. They tend to settle in certain sections of large cities, and must adjust to many new patterns of living. This study will relate:

(A) Linguistic, non-linguistic, and total I.Q. and achievement in five school subjects as measured by standardized tests with: . (a) 6 Fels Parent Behavior Ratings measured by home interviews; (b) 20 indices of socioeconomic standing; (c) 5 measures of family mobility; and (B) teacher and pupil sociometric ratings of school adjustment with (a), (b), and (c) above. Data in section (a) will be continuous variables. Intercorrelations of each variable in these sections will be computed by Pearson Product-Moment correlations. Most of the data in sections (b) and (c) will be dichotomous. Chi-squared or point biserial correlations will be used for these. Computed data correlations will indicate areas where school and community programs may concentrate on improving the well-being of these new urban residents. (Edwin V. Kelley)

2072-66023 T: Civil Engineering. Eulerian Calculations of Stresses and Motions in Compressible Solid Media. On the basis of a physical discrete model, the basic system of conservation equations is used for describing wavepropagation and rising of shocks in compressible media, according to the Eulerian standpoint of a fixed space-gridwork. Appropriate constitutive relations for elastic-plastic compressible material behavior are used together with the conservation relations, allowing for an evaluation of both hydrostatic and deviatoric effects in the stress waves. The system of difference-equations resulting from the discretization of the continuum is numerically integrated through an iteration procedure hinging on the conservation of momentum equation and on the structure of the material derivative of the relevant physical quantities. Present work is concerned specifically with the dynamical expansion of spherical cavities in an infinite granular medium due to high intensity pressure pulses. (E. Faccioli) 
2073-66024 T Civil Engineering. A Stochastic Model for Rainfall-Runoff Process. The central problem of this research is to develop an appropriate model for the transformation of rainfall to runoff. A historical record of total rainfall and runoff will constitute the basic data of the problem. The time series of these two hydrologic events will be considered as two stochastic processes. Statistical analysis of the two time series will be made to determine covariance, spectrum, and other statistical parameters of the series. These statistical characteristics will help determine the probability laws and nature of the stochastic processes. Knowing these, an attempt will be made to devise a stochastic model which appropriately represents the transformation of the stochastic process represented by the rainfall time series to the one represented by the runoff time series. Several modifications might have to be made before a satisfactory model is arrived at. This model will be used to generate synthetic runoff data from sequentially generated rainfall data. (T. Prasad)

2074-66025 Agricultural Engineering. Farm Machinery Operations Analysis. The objective is to select a tractor-implement system for a specified condition using least-cost as a criterion. Existing optimum selection equations permit selection. for either an optimum power level or for a least-cost selection of individual implements. The purpose of this study is to inter-relate both approaches with least-cost of the whole system as the criterion. The program first selects an optimum power level for given conditions, and then completes the system by sizing. implements to that power load. Four new power loads are examined on each side of the apparent optimum, and the related system costs are examined for a minimum. (D. R. Hunt)

2075-66026 T Aeronautical and Astronautical Engineering. Compressible Mixing Region Analysis: The problem involves the turbulent mixing region between two parallel, compressible streams of a gas. The computer will be used for: (1) obtaining numerical solutions to the differential equations of motion (ordinary differential equations); (2) to evaluate parameters that must be determined through use of the experimental data; and (3) to perform repetitious calculations representing different values of the flow variables. (V. P. Roan) 
2076-66027 Survey Research Laboratory. Readership. This project involves study of readership information concerning a professional marketing journal. The data was obtained by mail and telephone. Frequency counts, multiple regression analyses, and chi square designs.will be run. (R. Gunnigle)

2077-66028 Division of General.Studies L A S. Choice, Obligation, Dogmatism and Attitude Change. The hypotheses of this experiment are derived, in part, from Festinger's theory of cognitive dissonance. The subjects were pretested on their attitudes toward several topics of national importance two weeks in advance of the experiment, and a.topic was selected on which all subjects were in relative agreement. At the time of the experiment, the subjects were asked to listen to a speech in which an opinion contrary to their private. beliefs were expressed. Subjects had been assigned to one of eight conditions in a $2 \times 2 \times 2$ factorial design. Each subject was assigned to a high or low dogmatic group on the basis of scores on Rokeach's Dogmatism Scale, to a high or low choice condition, and a high or low obligation condition (in the high obligation group, reasons were given why he ought to comply by listening to the speech). Subjects received a post-test following the playing of the tape recorded speech. The change scores for each subject will be evaluated by means of analysis of variance. It. is predicted that choice and obligation will interact and that dogmatism will interact significantly with the levels of choice and obligation (3-way interaction). (Stanley E. Jones)

2078-66029 T Electrical Engineering. Optimal Satellite Control. A reaction wheel system is to control the orientation of a space vehicle. The optimal control function to be determined is to satisfy certain constraints, one of which minimizes the integral of the power required by the motor ariving the reaction wheel. The computer will be used to evaluate the integrals and to select the appropriate sequences of the control. (G. E. Bergmann)

2079-66030 Psychology. Stimulus-Response Inventory of Traits. An important and recurrent issue in personality theory is whether the major source of variance in behavior derives from the person or from the situation. 
Personologists: have assumed that individual differences constitute the major source of behavioral variation while social psychologists have emphasized the primacy of situational factors. An analysis of the Stimulus-Response Inventory of Anxiousness data suggests that the debate over the relative importance of individual differences and of situations is largely a pseudoissue. Variance components derived from the mean squares of three way classification analyses of variance indicate the subjects ( $\underline{S} s$ ) account for about $5 \%$ of the total variation (sum of the variance components), situations account for about 5\%-12\% of the variance, modes of response for about onequarter to one-third of the variance, and that nearly a third of the variance comes from the three simple interactions (모' $x$ situations, $\underline{\text { Ss }} \mathrm{x}$ Modes of Response, Situations $\times$ M-R). These proportions are highly stable across samples of subjects there the same form of the inventory has been used. The aims of the program are: (I) To continue the development of the inventory of anxiousness to permit more accurate description of individuals in terms of appropriate categories of responses to appropriate categories of situations. The computer will be used to factor analyze the subjects' modes of response and situational scores. (2) To ascertain the generality of the findings from our previous studies across various modifications of the samplings of subjects, across various modifications of the samplings of situations and of modes of responses. Three way analyses of variance will be computed and variance components will be derived from mean squares. (3) To explore the validity of inventory reports of various autonomic responses in terms of actual autonomic responses to various kinds of situations. It may be feasible to "digitize" and tape record the autonomic responses (derived in analogue form from a polygraph) so that they can later be analyzed on the electronic computer of the Coordinated Science Laboratory at the University. (4) To determine if the principles of personality description discovered with the various S-R inventories of anxiousness. will hold with the trait of anger. The S-R anger situational and mode of response scales will be factor analyzed. Three ways analyses of variance will be computed on the raw data and variance components will be derived from the mean squares. This will enable us to determine the relative contribution of situational, subject, mode of response, and interactions to the total. behavioral variation... (Norman Endler) 
2080-66031 T Honors. Study of Certain Variables Related to Attrition Rate of James Scholars. This problem involves. James Scholar Freshmen and some of the variables related to their remaining in and dropping from the James Program. The computer will be used to compute correlation coefficients, t-tests, and an analysis of variance for scores earned on certain measures of creativity and status within the program at the end of the freshman year. Tests and questionnaires provided the measures of creativity to be used. This work is a. replication of studies performed by Getzels and Jackson (at the University of Chicago) and by Torrance (University of Minnesota). (Robert Griffin)

2081-66032 Aeronautical and Astronautical Engineering. Convective Discharge. This work is part of a theoretical investigation of convective electric discharge phenomena. Mathematical models for the convective discharge are to be analyzed; including computation of the jump conditions and Hugoniot curves for the ionizing front. Standard subroutines (probably including UTR2) will be used. Results of the Hugoniot calculation will provide insight into the properties of ionizing fronts for a range of values of Lorentz force and Joule heating. (C. E. Bond)

2082-66033 Material Research Laboratory. Radiation Damage in Crystals. This research project involves the study of point defects in nearly perfect silicon, germanium, and copper crystals. The defects are produced at $40 \mathrm{~K}$ by electron bombardment using a 3 Mev Van de Graaff accelerator. Anomolous transmission of $x-r a y s$ (the Borrmann effect) will be used to determine the concentration and type of point defects present at temperatures from $4^{\circ} \mathrm{K}$ to $300^{\circ} \mathrm{K}$. The computer will be used to compare the experimental integrated intensity with equations based on the dynamical theory of x-ray diffraction. (Dr. James S. Koehler) 
2083-66034 T Physical Education for Men and Graduate. PE. Factors Influencing Total. Peripheral: Resistance Among Boys. The casual analysis will be used in: an attempt to determine which variables make a definite contribution to total peripheral resistance among boys. Subjects will be boys whose ages range from 8 to 14. who participated in the Sport. Fitness program, and also some 13 and 14 year old boys from Urbana Junior High School. The chi. square technique for goodness. of fit will be employed. The Doolittle correlation will be used to establish data weights. (Roy D. Moore)

2084-66037 Institute for Research on Exceptional Children: Prevocational Program for Drop-Out-Prone Children. This project is concerned with the evaluation of a special prevocational program for culturally deprived, dropoutprone youth of dull intellectual ability. Approximately two-hundred youths were identified, placed in special classes, and provided with a special curriculum. Objective measures of academic achievement, aptitude, and various socio- and emotional factors. were obtained on a pre- and post-test basis for all available subjects. Appropriate statistical methods including correlation, analysis of variance, and possibly factor analysis are to be used to evaluate the data, depending upon the characteristics of the attained scores. Computer techniques seem appropriate because of the number of subjects and the large number of variables. (Merle B. Karnes)

2085-66038 T Psychology. Learning Under Stress. The research deals with the roles of test anxiety and self-esteem in children's verbal learning. Data were collected in a classroom situation. An intercorrelation matrix, using SSUPAC, will be run on all of the subject variables: test anxiety, self-esteem, IQ, and learning data. Subsequently, analyses of variance.will be run on various data fields. Results. will be used to supply information about some of the important motivational variables in. learning and to guide further research along these lines, with the hope of eventually providing help to both researchers and educators. (Dorothy Lekarczyk) 
2086-66039 T Chemistry and Chemical Engineering. Rotational: Energy Transfer. The overall project is the study of rotational-translational energy exchange in bimolecular collisions. Approximate solutions to the equations of motion for the problem of a rigid diatomic molecule colliding with an atom will be examined. The computer will be used for numerical evaluation of the integrals obtained. The results will be compared with recent experimental results for molecular beam studies of bimolecular collisions. (Audrey Cohen)

2087-66040 Psychology. Cognitive Development in. Infants. In the present project, the aim. was to measure cognitive development in infants from homes of adequate and inadequate stimulation value; within each group, five separate age levels were run. The cognitive development data was obtained through testing each child on selected sub-scales of the Hunt-Uzgiris Scale of Infant Psychological Development. The stimulation value of the home was measured through assessing each child's home environment on a modification of the Caldwell Inventory of Home Stimulation Value. The computer will be used to run point-biserial correlations between each item of each child's performance on the Psychological Development Scale and each item of the Home Stimulation Scale for that particular child. Assuming a matrix. with sufficient variation were obtained, a principle axis factor analysis solution with verimax rotation would also be run on the above data. The data obtained would be studied in an attempt to determine what aspects of home stimulation are most important in influencing cognitive development in children. (Theodore D. Wachs)

2088-66041 T Psychology. Comparison Factor Analyses of Correlations and Cross. Products. This project consists of an examination and comparison of results of various methods of structuring behavior change from occasion to occasion. Computer analyses will consist. of various transformations of the empirical data, e.g. standardization of scores, followed by application of factor analytic methods to the matrices of cross products and correlations. The empirical data consist of two sets of physiological and psychological measures obtained on (a) a sample of 94 university undergraduates, and (b) a sample of 147 junior high school students. Both samples were measured twice with the same instruments with a lapse of a week to a month between testing occasions. (Nesselroade) 
2089-66042 T. Political. Science. Measuring Orientation Towards Authority • This problem is concerned with the construction and validation of a scale to measure a person's "orientation towards authority". An operational measure of this concept would allow the testing of a number of theories in various areas of political. science. The data is obtained through questionnaires administered to both student and non-academic subjects. The computer will be used to perform factor analyses, correlational analyses, and other statistical analyses of the data. The computer results. will be used to refine and validate the scale. (Fred Herzon)

2090-66045 T Psychology. Trial By Trial Concept Attainment. The project is concerned. with an investigation of how adults learn simple concepts. More specifically, special attention. is given to trial by trial verbalizations of the subjects, concerning their. hypotheses of the correct concept. The data was obtained in twelve two hour sessions using the PLATO System. At each session eighteen subjects.worked on two simple concepts. Twenty dependent measures. were derived from the raw data. (the record of the subject's performance): Correlation matrices for each subject over trials will be computed. In addition, analysis of variance will be computed on these variables using type of problem and method of presentation of the stimuli as independent variables. These results will be evaluated in terms of a theory of propositional control of behavior. (Steven Schwartz)

2091-66046 T Agricultural Economics. Economics of Dairy Management Systems. Linear programming: by the simplex procedures will be used to select the optimum forage harvesting techniques when labor is restricted and given farm facilities are assumed. Data.will be obtained from research reports of the departments of Agronomy, Dairy.Science, and Agricultural Economics. The computer ressults.will be used to recommend the method of harvesting. to be used when certain assumed conditions are met. (Thomas L. Coltman) 
2092-66047 T Psychology. Abilities, Transfer, and Information Retrieval in Verbal Learning. This study is a multivariate experiment designed to test a theory relating multiple ability and information processing predictor variables to parameters describing learning performance. An organizability dimension is manipulated for the verbal materials learned by the subjects, and hypotheses of two kinds are to be tested: (1) hypotheses concerning the structure of the abilities, strategies, and performance domains; and (2) hypotheses concerning differential predictability from the predictor variables to the learning parameters under different treatment conditions. The data are obtained from testing 150 high school students in two sessions. In the first session, scores on 14 ability tests are obtained from testing 150 high school students; in the second, the subjects learn a 60 word list in 18 recall trials and, after the 18 trials are complete, respond to a Strategy Assessment Test designed to measure their use of 16 different retrieval mechanisms. The statistical analyses, to be accomplished on the computer, involve: (1) the determination of generalized learning curves by factor analysis; (2) rotation to a simple structure hypothesis; (3) maximum likelihood factor analysis; (4) multivariate analysis of variance; and (5) multiple regression and canonical correlation analysis. Procedures (1) to (3) will test structure hypotheses; (4) and (5) will test hypotheses of differential predictability and central tendency. (C. H. Frederiksen)

2093-66048 Chicago Circle Psychology. Factors Associated with Academic Performance. An investigation is being performed of intellectual and nonintellectual factors associated with performance in particular courses or sets of related courses. An eight hour battery of tests covering a variety of general intellectual skills has been administered to a representative sample of University freshmen. Item analyses.and factor analyses will be performed to improve instrument reliability and to define more clearly the skills involved. Multiple regression analyses will then be performed to determine the relative effect of each skill identified upon performance in each of about 15 courses typically taken by incoming freshmen. Supplementary analyses will be performed to determine the linearity of these 
relationships. An investigation of certain nonintellective correlates of academic achievement will be conducted. similarly. Results should provide guidelines for the development of special educational programs designed. to increase the likelihood of students' ultimate academic success. (Robert. Wyer)

20.94-66049 Home Economics. A. Study of Illinois Consumers' Reactions to Use of Pest Control on. Agricultural Products. The over-all objective is to obtain insight concerning the Illinois consumers' knowledge of, attitudes toward, acceptance of, and demand for agricultural products subjected to varying degrees and alternative methods. of pest control. Specific objectives of the project are as follows. For a representative sample of consumers: 1) to study the relationship between knowledge of and attitudes toward the use of agricultural products subjected to pest control; 2) to study not only the attitudes. but also the strength of these attitudes toward the use of agricultural products subjected. to pest control; 3) to study the relationship between knowledge of and acceptance of the use of agricultural products subjected to pest control; 4) to study the relationship between attitudes toward and acceptance of the use of agricultural products subjected to pest control; 5) to study the relationship between attitudes toward and demand for agrịcultural products subjected to pest control. In addition. to using the computer to obtain the frequency distribution for each of the 77 questions on the questionnaire, significance using. chi square will be determined for selected statements after classification by family characteristics such as education of wife, occupation of husband, area. in. which raised, etc. The relationships between attitudes, knowledge, and demand of families for agricultural products subjected to pest control will be determined. A random sample of families. in. Champaign-Urbana was obtained using the latest issue of the City Directory. The sample consists of 704 usable family records. The Federal Government is very concerned. with determining people ${ }^{i}$ s attitudes toward agricultural products subjected to pest control. It is anticipated that the information' from this study will be used to determine educational programs and legislative action. (Dunsing) 
2095-66050 T Physical Equcation for Women. A study of. Women Athletes. The computer will be used to determine significant differences among three groups of women athletes, in twenty-seven structural and functional measurements. The F ratio for analysis of variance will be used to determine significant differences. In addition, Pearson Product-Moment formula will be used to determine the accuracy. (or reliability) of the testers. The data were obtained from a test battery of twenty- seven items administered to forty subjects, all undergraduate women physical education majors at the University of Illinois.: There were fourteen subjects representing field hockey, twelve representing gymnastics, and fourteen representing tennis. The computer results will be used to test the hypothesis that women athletes in one activity differ structurally and functionally from women athletes in another activity. (Cohen)

2096-66051. T Mining, Metallurgy, and Petroleum Engineering. Determination of Stress for Notched Regions. This research problem will consist of finding the stress distributions for elastic bodies having "notched" edges. This consists of solving the biharmonic equation, $\nabla^{4} \varphi=0$, by a relaxation scheme. This scheme does not use residuals, but assumes that the residuals are zero. The results obtained will be used as models for geological structures. For example, a shallow smooth notch can represent a valley. (John R. Sturgul)

2097-66052 Institute for Research on Exceptional Children. Culturally Disadvantaged Children. The purpose of this problem is to determine if culturally disadvantaged children between the ages of eight months and two years who are provided with a program of intellectual stimulation for one hour a day, five days a week, will reach at the age of four years a level of cognitive development which exceeded that attained by older untutored siblings when they were four years old. From a population of culturally deprived boys and girls assigned to pre-school classes, thirty siblings between the ages of eight months and two years will be randomly drawn and assigned to two groups of fifteen male and female subjects, one group receiving intellectual stimulation and the other not. Screening measures 
and assessment procedures include: Assessment of Stimulation Potential of Home, Tels Parent Behavior Rating. Scale, Index of Social Characteristics, Cattell's Infant Intelligence Scale, Stanford-Binet, Revised, Medical Examination, Instrument for Assessing Infant Psychological Development, Outline of Language Development, Outline of Motor Development. A Areas included in the training program are movement, self-concept, object manipulation, language, symbolic representation, and socialization. Statistical techniques employed to assess the value of the training program include: T-tests to evaluate the significance of $I Q$ changes, and analyses of covariance to investigate effects of experimental treatment and various environmental and behavioral variables. The relationship between medical, biographical, and home stimulation variables and gains in $I Q$ will be determined by correlation with residual gains. Data will be used to provide information on training programs, to suggest the appropriate time for introduction of various toys and play activities, and to trace cognitive development in children eight months to two years. (Will Beth Stephens)

2098-66054 Mechanical Engineering. Fuel Synthesis Studies. Thermodynamic analysis will be conducted to determine the range of purity obtainable in the synthesis of a high energy rocket fuel. Comparative calculations will be made to determine specific impulse obtainable with synthetic and standard fuel systems. Resultant data will be presented in tabular and graphical (CalComp plotter) form. (W. E. Bair)

2099-66057 Department of Computer Science. Integer Linear Program. A computational procedure has been developed to synthesize a threshold element network for a given switching function. One can include in this algorithm such network restrictions, as input tolerance, maximum fan-outs, and maximum number of variable inputs to any element. Since little is known about computational efficiency of integer linear programming, various techniques will be tested. Once a reasonable integer linear programming code is operational, it will be used to synthesize networks for various switching functions. (Saburo Muroga) 
2100-66058 Provost's Office. Teaching Services - Productivity Study. Simultaneous equation models describing the production, output demand, and factor demands for general industrial divisions (sectors) will be estimated. over the states using $\mathrm{k}$-class procedures in SSUPAC. These estimating equations are then to be used in evaluating Illinois industrial performance. (D. Aigner) 


\subsection{Instructional Problem Specifications}

During the second quarter of 1966, 4 instructional problem specifications were submitted to the Department for computation. The following brief descriptions of these problems have been prepared for inclusion in this report by those submitting them:

I374-64002 Electrical Engineering 498. Sensitivity Functions. It is desired to determine the sensitivity of a multivariable control system to parameter variations. Sensitivity functions will be computed using a Midas analog simulation of the system on the digital computer. (David M. Salmon)

I379-64043 Nuclear Engineering 495. Monte Carlo Studies. The problem is a study of the Monte Carlo method as applied to gamma ray transport. The purpose is to give training in the gamma ray transport processes and to give training in the handling of such processes by modern day Monte Carlo methods used on computers. (A. B. Chilton)

1381-64048 Theoretical and Applied Mechanics 311. Mechanical Vibrations. Theoretical and Applied Mechanics 311 class problem. (W. J. Worley)

I383-64058 Nuclear Engineering 495. Relative Intensities of Spectral Lines. The relative intensities of spectral lines as a function of temperature and electron density will be calculated and plotted for various spectral lines of neon, using data and equations from a recent text by Griem. The curves obtained may be used for measuring plasma temperatures from the relative intensities of spectral lines observed. (Goldstein) 


\subsection{Blanket Class Problem Specifications}

During the second quarter of 1966, 21 problem specifications were submitted to cover all assigned problems in the following courses:

J375-64007 Graduate School of Business Administration 544.

J376-64012 Psychology 332.

J377-64020 Electrical Engineering 497.

J378-64021 Electrical Engineering 359.

J380-64033 Civil Engineering 361:

J382-64056 Theoretical and Applied Mechanics 294.

J384-64062 Economics 471 .

J385-64070 Civil Engineering 461.

J386-64071 . Civil Engineering 263.

J387-64072 Civil Engineering 263.

J388-64079 Civil Engineering 316.

J389-66014 Civil Engineering 497.

J390-66017 Mechanical Engineering 263.

J391-66018 Mechanical Engineering 264.

J392-66035 Mathematics 387 .

J393-66036 Chemistry and Chemical Engineering 343 . 

J394-66043 Mathematics 195.
J395-66044 Department of Computer Science 996.
J396-66053 Electrical Engineering 323.
J397-66055. Industrial Education 459.
J398-66056. Nuclear Engineering 458. 


\section{GENERAL IABORATORY INFORMATION}

\subsection{Personnel.}

The number of people associated with the Laboratory in various capacities is given in the following table:

$\begin{array}{lccc} & \begin{array}{c}\text { Full- } \\ \text { time }\end{array} & \begin{array}{c}\text { Part- } \\ \text { time }\end{array} & \begin{array}{c}\text { Full-time } \\ \text { Equivalent }\end{array} \\ \text { Faculty } & 15 & 1 & 15.5 \\ \text { Visiting Faculty } & 3 & 3 & 4.5 \\ \text { Research Associates } & 1 & 0 & 1.0 \\ \text { Graduate Research Assistants } & 5 & 62 & 35.75 \\ \text { Graduate Teaching Assistants } & 0 & 1 & .12 \\ \text { Professional Personnel } & 12 & 1 & 10.75 \\ \text { Administrative and Clerical } & 17 & 1 & 17.5 \\ \text { Other Nonacademic Personnel } & \frac{73}{124} & \frac{93}{162} & 108.43 \\ \text { TOTAL : } & \frac{13}{19} & & 193.54\end{array}$

The Computer Advisory Committee consists of Professor J. R. Pasta, Head of the Department; Professor J. N. Snyder, Associate Head of the Department; Professors L. D. Fosdick, H. G. Friedman, C. W. Gear, D. B. Gillies, N. T. Hamilton, D. J. Kuck, B. H. McCormick, S. Muroga, T. A. Murrell, J. Nievergelt, W. J. Poppelbaum, S.R. Ray, J. E. Robertson and D. E. Slotnick. 


\subsection{Bibliography}

During the quarter, the following publications were issued by the Laboratory .

File Numbers

[1] Haas, M., E. Hassler, 'R.. Fisher, and C. Shepard, "Comments on Slow Speed, Hard Copy Remote Terminals," File No. 695, May 5; 1966.

[2]. Kato, Masao; "Arithmetic Array: A Computer Organization With Integrated Circuits," Filw No. 698, June 1, 1966.

[3] Mullins, W. E., "A Microminiature Flag Type Indicator" File No. 692, April 1, 1966.

[4] Slotnick, D. L., "ILLIAC IV Design Questions: Preliminary List," File No. 693, April 20, 1966.

[5]. Squires, B. E., "Storage Allocation Specification in Multiple Pass Compiling Systems," File No. 694, May 5, 1966.

[6]' Squires, B. E., "Precedence Grammars and the EULER System. Part I: Further Examples on Methods for Constructing Precedence Grammars," File No. 696, May 18, 1966.

[7] Squires, B. E., "Precedence Grammars and the EULER System. Part II: An Algorithm for Deciding Upon the Existence of Precedence Functions :and for their Computation," File No. 697, May 20, 1966.

\section{Report Numbers}

[1] McCormick, Bruce H., and A. M. Richardson, (Architectural Design), "Design Concepts for an Information Resource Center with Option of an Attached Automated Laboratory," Report No. 203, May 1, 1966.

Theses

[1] Bayer, Rudolf, "Automorphism Groups and Quotients of Strongly Connected Automata and Monadic Algebras," (Ph.D:), Report No. 204, May 18, 1966.

[2] Wattman, Walter J., "Multivibrator Triggering Requirements," (M.S.), Report No. 202, April 15, 1966. 


\section{Specification Numbers}

[1] Casasent, D. P. and D. S. Sand, "Specifications for Laser System," File No. 550-79, May 31, 1966.

[2] Divilbiss, J. L., "Specifications for Slow Silicon Multiple Junction Diodes Used for Level Shifting, "File No. 550-70, April 18, 1966.

[3.] Divilbiss, J. L., "Specifications for 200 MA Silicon Switching Transistor (USN 43)," File No. 550-72, May 12, 1966.

[4] Divilbiss, J. I. and B. H. McCormick, "Specifications for Teletype Page Printing Consoles for use with the ILIIAC III Communication Net," File No 550-74, June 14, 1966.

[5] Koo, Tuh Kai, "Specifications for Pulse Generator," File No. 550-77; June 28, 1966.

[6] Kubits, W. J. and D. C. Rollenhagen, "Specifications for Multifunction Storage Unit," File No. 550-71, April 25, 1966.

[7] McCormick, B. H., "Specif,ications for High Resolution Closed Circuit Television Camers and Monitor System," File No. 550-73; June 13, 1966.

[8] McCormick, B. H., "Specifications for Linc-Type Magnetic Tape Modules," File No. 550-75, June 17, 1966.

[9] McCormick, B. H., "Specifications for a High Speed Electrostatic Page Printer for Graphical Alphanumeric and Facsimile Printing," File No. 550-78, June.16, 1966.

[10]. Ray, S. R., "Specifications for a Magnetic Core Memory for ILLIAC III ("Fast Core")," File No. 550-76, June 2l, 1966. 


\subsection{Numerical Analysis}

"The Algebraic Eigenvalue Problem - VI," by Professor Don Secrest, Department of Chemistry, University of Illinois, Urbana;--Illinois, April 7, 1966.

"The Algebraic Eigenvalue Problem - VII," by Professor Don Secrest, Department of Chemistry, University of Illinois, Urbana, Illinois, April 21, 1966.

"The Algebraic Eigenvalue Problem - VIII," by Professor:

N. T. Hamilton, Department of Computer. Science, University of Illinois Illïnois, Urbana, Illinois, April 28, 1966.

"The Algebraic Eigenvalue Problem - IX," by Professor N. T. Hamilton, Department of Computer Science, University of Illinois, Urbana, Illinois, May 5, 1966.

"The Algebraic Eigenvalue Problem - XI," by Mr. Leland McDowell, Department of Computer Science, University of Illinois, Urbana, Illinois; May 19; 1966. 


\section{$8: 4$ Colloguia}

"Large Scale Integration Technology and Its. Impact on System Design," by Dr. Richard L. Petritz, Director, Semiconductor Research and Development Laboratory, Texas Instruments, Dallas, Texas, April 4, 1966.

"Discrete Representations of Differential Operators," by Professor David M. Young, Jx., Director, Computer Center, The University of Tiexas, Austin, Texas, April 19, 1966.

"Automata. Theory as a Basis for Studies of Adaptation," by Professor John. Holland, Associate Professor Communication Sciences, University of Michigan, Ann Arbor, Michigan, April 25, 1966.

"Neural Modeling," by Dr. Leon Harmon, Bell Telephone Laboratories, Inc., Murray Hill, New Jersey, May 2, 1966.

"An' Online Interpreter for Computer Aided Instruction," by Dr: Ken Iverson, IBM Watson Research Center, Yorktown Heights, New York, May 16, 1966.

"Practical Computer Needs for Neutron Transport Calculations," by Professor Felix T. Adler, Department of Physics, University of Illinois, Urbana, Illinois, May 23, 1966. 


\subsection{Drafting}

During the quarter, a total of 655 drawings were processed by both drafting sections:

\section{General and ILLIAC II}

Large. Drawings

Medium Drawings

Small Drawings

Layouts

Report Drawings

Changes

Miscellaneous

Semiconductor Coding

TOTAL
15

172

41

0

0

2

5

$\frac{0}{235}$
Pattern Recognition

$\frac{7}{420}$

K. C. Law and L. A. Prendergast

\subsection{Shops' Production}

Job orders processed and completed during the first quarter of 1966, are as follows:

$\begin{array}{lccc}\quad \text { Facility } & \text { AEC 1018 } & \text { AEC 1469 } & \text { Other } \\ \text { Machine Shop } & 44 & 59 & 3 \\ \text { Electronics Shop } & 36 & 48 & 0 \\ \text { Etch Shop } & 25 & 39 & 2 \\ \text { Layout Shop } & 36 & 41 & 0\end{array}$

Wiring of 374 standard printed circuit boards during this period, accounted for 9,569 diodes and 2,905 transistors.

Frank P. Serio 